Aus der Fakultät für Medizin der Universität Regensburg Chefarzt: Privatdozent Dr. med. Dr. med. habil. Bert Reichert Plastische, Wiederherstellende und Handchirurgie

\title{
Der Nürnberger Handscore: Ein neues System zur Erfassung von Verbrennungsfolgen an der Hand
}

\author{
Inaugural-Dissertation \\ zur Erlangung des Doktorgrades der Medizin \\ der Fakultät für Medizin der Universität Regensburg
}

vorgelegt von

Beate Maria Schneider

2010 

Aus der Fakultät für Medizin der Universität Regensburg Chefarzt: Privatdozent Dr. med. Dr. med. habil. Bert Reichert Plastische, Wiederherstellende und Handchirurgie

\title{
Der Nürnberger Handscore: Ein neues System zur Erfassung von Verbrennungsfolgen an der Hand
}

\author{
Inaugural-Dissertation \\ zur Erlangung des Doktorgrades der Medizin \\ der Fakultät für Medizin der Universität Regensburg
}

vorgelegt von

Beate Maria Schneider

2010 
Dekan:

1. Berichterstatter:

2. Berichterstatter:

Tag der mündlichen Prüfung: $\quad 29.12 .2010$
Prof. Dr. Bernhard Weber

Privatdozent Dr. Bert Reichert

Prof. Dr. Hans Jürgen Schlitt 


\section{Inhaltsverzeichnis}

1.

EINLEITUNG

1.1. DIE BEDEUTUNG DER HAND

1.2. EINFÜHRUNG IN DIE THEMATIK 2

1.3. DIE VERBRENNUNGSVERLETZUNG $\mathbf{5}$

1.3.1. ANATOMIE UND PHYSIOLOGIE DER HAUT

1.3.2. PATHOPHYSIOLOGIE DER VERBRENNUNGSWUNDE 6

$\begin{array}{ll}\text { 1.3.3. EINTEILUNG DER VERBRENNUNGSTIEFE } & 7\end{array}$

1.3.3.1. Verbrennungen ersten Grades $\quad 8$

1.3.3.2. Verbrennungen zweiten Grades $\quad 8$

1.3.3.3. Verbrennungen dritten Grades 9

1.3.4. BEURTEILUNG DER FLÄCHENAUSDEHNUNG EINER
VERBRENNUNGSVERLETZUNG

1.3.5. THERAPIE VON VERBRENNUNGSWUNDEN 11

1.3.6. THERMISCH GESCHÄDIGTE HÄNDE 16

$\begin{array}{ll}\text { 1.3.7. LEBENSQUALITÄT } & 21\end{array}$

2. FRAGESTELLUNG 23

3. PATIENTEN UND METHODIK 25

3.1. EINSCHLUSSKRITERIEN 25

3.2. DATENERFASSUNG 25

3.2.1. RETROSPEKTIVE ANALYSE DER FALLDOKUMENTATION 25

3.2.2. DASH FRAGEBOGEN

3.2.3. NÜRNBERGER FRAGEBOGEN HANDVERBRENNUNG 27

$\begin{array}{lll}\text { 3.3. STATISTISCHE AUSWERTUNG } & \mathbf{2 8}\end{array}$

3.3.1. METHODIK 28

3.3.2. DER NÜRNBERGER HANDSCORE 29

$\begin{array}{lll}\text { 3.3.3. PLAUSIBILITÄTSANALYSE } & 31\end{array}$

4. ERGEBNISSE

4.1. ANALYSE DER BEHANDLUNGSFÄLLE 33

4.1.1. DESKRIPTIVE STATISTIK 33

4.1.1.1. Ursache der Verbrennungsverletzung 33

Tiefenausdehnung der Handverbrennung

DASH-FRAGEBOGEN $\quad 35$

4.2.1. DESKRIPTIVE STATISTIK

4.3. NÜRNBERGER FRAGEBOGEN 38

$\begin{array}{lll}\text { 4.3.1. DESKRIPTIVE STATISTIK } & 38\end{array}$

4.3.2. DEFINITION DES NÜRNBERGER HANDSCORES 41

4.4. RELIABILITÄTSANALYSE VON DASH-SCORE UND NFH-SCORE 45

4.5. PLAUSIBILITÄTSANALYSE 46

4.6. VERGLEICH ZWISCHEN DASH-SCORE UND NFH-SCORE 48

4.6.1. KORRELATIONSANALYSE 48

4.6.2. STATISTISCHER VERGLEICH DER BEIDEN SCORES 49 
5. DISKUSSION 52

5.1. DASH-SCORE 54

5.2. NÜRNBERGER HANDSCORE

5.3. INTERPRETATION 57

$\begin{array}{lll}\text { 5.4. } & 59\end{array}$

6. $\quad$ ZUSAMMENFASSUNG 61

7. $\quad$ LITERATURVERZEICHNIS $\quad 62$

8. $\quad$ ANLAGEN $\quad 68$

$\begin{array}{ll}9 . & 73\end{array}$

10. LEBENSLAUF 74 
Abkürzungsverzeichnis

\begin{tabular}{|l|l|}
\hline \%vKOF & Anteil der verbrannten Körperoberfläche in Prozent \\
\hline ABA & American Burn Association \\
\hline NFH & Nürnberger Fragebogen Handverbrennung \\
\hline NFH-Score & Nürnberger Handscore \\
\hline $2 a$ & Oberflächliche zweitgradige Brandverletzung \\
\hline $2 b$ & Tief zweitgradige Brandverletzung \\
\hline 3 & Drittgradige Brandverletzung \\
\hline ABSI & abbreviated burn severity index [58] \\
\hline
\end{tabular}




\section{Einleitung}

\subsection{Die Bedeutung der Hand}

Die Entwicklung der Hand zu einem komplexen Greif- und Tastorgan war wesentliche Voraussetzung für die evolutionäre Menschwerdung. Die Aufnahme von Sinnesinformationen in der Hand durch periphere Nerven, das Zusammenspiel des sensomotorischen Systems und schließlich die Verarbeitung der Informationen im zentralen Nervensystem spielen eine enorm große Rolle bei der Auseinandersetzung mit der direkten Umwelt. Der hohe Stellenwert der Hand lässt sich eindrucksvoll an der Größe des entsprechenden Hirnareals ablesen (Homunculus).

Die Hand ist sowohl Werkzeug als auch Wahrnehmungsorgan und erfüllt somit zunächst eine funktionelle Aufgabe. Durch die Sensibilität im Fingerkuppenbereich lassen sich Informationen ertasten und präzise Greifformen und Bewegungen steuern und umsetzten. Durch die Möglichkeit der Opposition des Daumens kommt es zur Erweiterung der Bewegungsmöglichkeiten und Verbesserung der Kraftentwicklung. Störungen oder Defekte an Daumen und Fingern führen folglich zu einem Verlust präziser Greiffähigkeit, einfache sekundäre Greifformen treten an ihre Stelle. Ein nichtsensibler Handanteil kann nur unter visueller Kontrolle für elementare Greifformen eingesetzt werden. Finger ohne Feingefühl sind „blind“. Auch Einschränkungen der Beweglichkeit an Fingergelenken können zu einer schwerwiegenden Beeinträchtigung der Funktion der ganzen Hand führen.

Auch unter sozialen und gesellschaftlichen Aspekten nimmt die Hand einen hohen Stellenwert ein. Der Gesamteindruck einer Person wird stark von der Erscheinung ihrer Hand beeinflußt. Oft wird ihre soziale Stellung dadurch hervorgehoben. Die Hand ist im Alltag ständig sichtbar und wird daher gern als Ausstellungsfläche für Schmuck genutzt.

Schlussendlich ist die Hand auch ein Mittel der Kommunikation. Typische Bewegungen symbolisieren über verschiedenste Kulturen hinweg eine global verständliche nonverbale Ausdrucksform. So hat beispielsweise die 
geballte Faust überall die gleiche Bedeutung. Die Persönlichkeit des Individuums zeigt sich auch in der Weise, wie diese Form der Kommunikation genutzt wird.

\subsection{Einführung in die Thematik}

Die "Deutschsprachige Arbeitsgemeinschaft für Verbrennungsbehandlung" erhebt jährliche Statistiken über die Patienten, die in den deutschsprachigen Zentren für Schwerbrandverletzte behandelt wurden [7]. Schätzungsweise kommt es allein in Deutschland jährlich zu etwa 480.000 leichten Verbrennungsverletzungen, 16.000 davon sind klinisch behandlungspflichtig, etwa 1.400 bis 1.900 Patienten mit schwersten Brandverletzungen müssen in eine Spezialklinik eingewiesen werden [38, 61]. Die durchschnittlich verbrannte Körperoberfläche liegt in diesen Fällen bei knapp 20\%, der mittlere ABSI-Score beträgt 6 [53]. Hauptursache für Verbrennungsverletzungen sind Haushaltsunfälle, gefolgt von Arbeitsunfällen, Verkehrsunfällen sowie Suiziden [62].

Ursachen für Verbrennungsverletzungen sind offene Flammen, Verbrühung mit heißen Flüssigkeiten, Kontakt mit heißen Oberflächen sowie direkte und indirekte Einwirkungen von elektrischem Strom. Aufgrund der Ähnlichkeit der Verletzungsfolgen werden auch chemische Verletzungen durch Säuren oder Laugen wie Brandverletzungen betrachtet [17].

Brandverletzungen hinterlassen fast immer Folgen, die mit einem hohen Leidensdruck einhergehen. Es resultieren dabei sowohl Funktionseinschränkungen als auch ästhetische Entstellungen, die eine erhebliche Minderung der Lebensqualität bedingen. Die am häufigsten betroffenen Körperteile bei Verbrennungsverletzungen sind die Hände. Sie werden in reflexartiger Abwehr zum Schutz gegen die Hitzequelle eingesetzt und sind dabei fast immer ungeschützt. Verbrennungsfolgen an den Händen haben häufig größere Konsequenzen für die Betroffenen als Verbrennungen an anderen Körperregionen, selbst wenn diese deutlich großflächiger sind. Funktionseinschränkungen der Hände können existentiell relevante Auswirkungen auf die Zukunft dieser Patienten haben, vor allem bei 
Handwerkern. Die Qualität der Therapie von Verbrennungsverletzungen, und zwar speziell von Handverbrennungen, hat eine wesentliche volkswirtschaftliche Bedeutung. Einerseits sind die Behandlungs- und Rehabilitationszeiten lang, zum anderen sind auch die Behandlungsergebnisse für das Wiedererlangen der Arbeitsfähigkeit sowohl hinsichtlich der physischen als auch der psychosozialen Folgen bedeutsam [12]. In der Öffentlichkeit führen sichtbare Entstellungen der Hände zu spezifischen Belastungssituationen [3, 15]. Die Belastbarkeit der Haut im Bereich der Hände entscheidet letztlich darüber, ob die Arbeitsfähigkeit wieder erlangt werden kann $[8,56]$.

Auch noch lange Zeit nach einer Akutbehandlung finden Heilungsvorgänge statt. Daher sind regelmäßige und qualifizierte Nachuntersuchungen sinnvoll. Es ist nicht ungewöhnlich, dass sekundäre Korrekturmaßnahmen erwogen werden müssen. So können beispielsweise Bewegungsbehinderungen durch narbige Kontrakturen operativ angegangen werden. Hierbei lassen sich einfache plastisch-chirurgische Methoden anwenden oder auch Hautverpflanzungen durchführen, falls dies erforderlich ist. Gelegentlich zeigen sich ehemalige Brandverletzungen nicht ausreichend stabil abgeheilt. Insbesondere an den Händen entstehen immer wieder an denselben Stellen offene Wunden. Schließlich ist zu erwähnen, dass sekundäre Rekonstruktionen bei schwerwiegenden Defektverletzungen erwogen werden müssen. Kommt es beispielsweise zu Fingerverlusten durch einen Stromunfall, ist im Verlauf zu prüfen, inwieweit die Handfunktion durch rekonstruktive Maßnahmen, z.B. die Verpflanzung von Zehen an die verletzte Hand, eine Verbesserung der Funktion ermöglichen kann. Kennzeichnend für diese Patienten ist dabei, dass sie durch die entstandenen Schäden unterschiedlich beeinträchtigt sind. Für einen Pianisten wird der Verlust eines Kleinfingers eine andere Auswirkung haben als für einen Handwerker oder für einen Rentner. Will man das individuell bestehende Maß der Beeinträchtigung erfassen, muss man also auch subjektive Einschätzungen berücksichtigen. 
Aus diesen Überlegungen wird deutlich, wie wichtig regelmäßige Befragungen und Nachuntersuchungen dieser Patienten sind. Bei der Erfassung der Befunde ist von besonderer Bedeutung zu erkennen, ob sich im zeitlichen Verlauf Änderungen ergeben haben.

Verletzungsfolgen an der oberen Extremität werden üblicherweise durch den seit langem etablierten DASH-Fragebogen erfasst [6, 18, 23]. Der DASH-Score wird als der am besten geeignete Score bei Patienten mit multiplen Funktionsstörungen der oberen Extremität betrachtet [10]. Der Patient selbst beantwortet strukturierte Fragen zu funktionellen Einschränkungen wie auch zu Einschränkungen in der Lebensqualität. Diese Selbsteinschätzung des Patienten ist trotz grundsätzlich denkbarer Täuschungs- und Fehlermöglichkeiten allgemein anerkannt. Wichtige Aussagen über spezifische Verletzungsformen, aber auch über Behandlungsmethoden, werden ebenso wie Prognosen zu Rehabilitation und Wiedereingliederung in das Berufsleben daraus abgeleitet.

Der DASH-Fragebogen gliedert sich in drei Abschnitte. Teil A besteht aus 23 Fragen bezüglich der Bewältigung alltäglicher Tätigkeiten, Teil B fragt 7 verschiedene Symptome ab und Teil $\mathrm{C}$ dient der speziellen Evaluierung von Einschränkungen bei Musikern und Sportlern.

Jede Frage wird anhand einer Skala von 1 bis 5 beantwortet. Das Ergebnis („DASH Score“) ist der Quotient aus der um 30 reduzierten Gesamtpunktzahl und der Bandbreite 1,2. Ergibt sich für jede Frage nur ein Punkt, so ist der ermittelte Score 0, was einer optimalen Funktion ohne Behinderung entspricht. Führen die Antworten andererseits für jede Frage zu einem Punktwert von 5, so ergibt sich ein Score von 100, was einer völligen Gebrauchsunfähigkeit der Hand entspricht. Die individuell ermittelten Scores bewegen sich also immer zwischen 0 und 100.

In seiner allgemein gehaltenen, auf die gesamte Bandbreite möglicher Verletzungen der oberen Extremität bezogenen Form geht der DASHFragebogen also nicht speziell auf Verbrennungsverletzungen ein. Ein 
vergleichbares Messinstrument für diese Verletzungsfolgen existiert bislang nicht.

\subsection{Die Verbrennungsverletzung}

\subsubsection{Anatomie und Physiologie der Haut}

Die Haut ist das größte Organ des Menschen. Sie bewahrt den Organismus vor Flüssigkeitsverlusten und schädigenden Umweltreizen, dabei bildet ihr Säureschutzmantel eine natürliche Barriere gegen Krankheitserreger. Sie steuert die Temperaturregulation und die taktile Wahrnehmung [60]. Histologisch wird die Haut in Epidermis, Dermis und Subcutis unterteilt (Abb. 1).

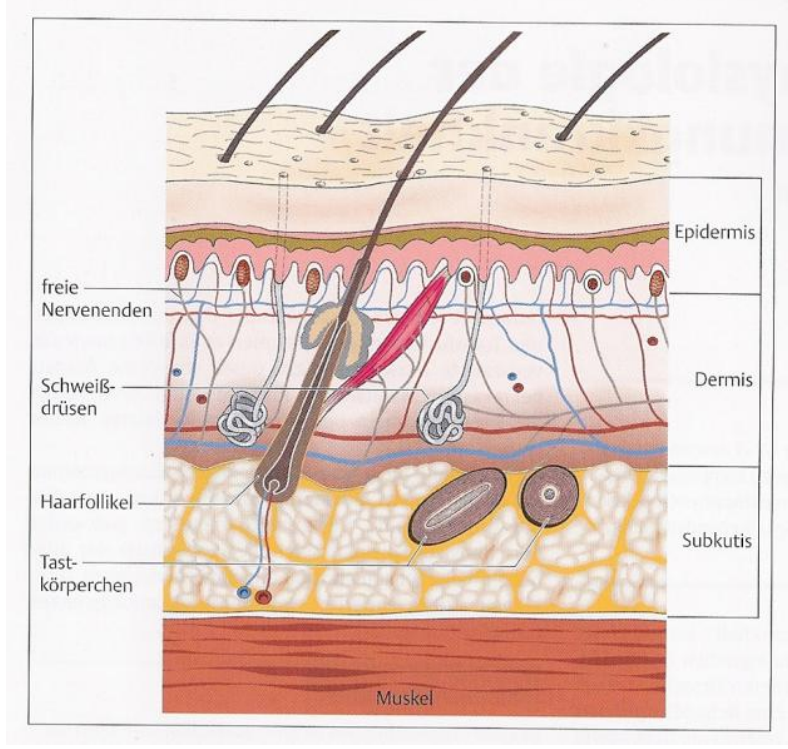

Abb. 1: Der Aufbau der Haut (aus [60])

Als Hautanhangsgebilde werden Haare, Nägel, Duft-, Talg- und Schweißdrüsen bezeichnet. Im oberen Anteil der an die Epidermis grenzenden Dermis (Stratum papillare) liegt ein subpapilläres Gefäßnetz. Daraus entspringen Blutkapillaren, die in die Papillen ziehen, wo auch Nervenfasern vorhanden sind. Der darunter liegende Anteil der Dermis (Stratum reticulare) ist deutlich derber und grobfaseriger. Diese Schicht ist für die mechanischen Eigenschaften der Haut von ausschlaggebender 
Bedeutung. Die Haarwurzeln und Schweißdrüsen der Haut ragen bis in die obere Subcutis hinein. Die Epidermis (Oberhaut) kann sich nach Verletzungen narbenfrei regenerieren. Die darunter liegende Dermis und Subcutis, die ohne scharfe Grenzen ineinander übergehen, lassen nach Verletzungen Narben entstehen. Die Dicke der Haut variiert je nach Körperregion.

\subsubsection{Pathophysiologie der Verbrennungswunde}

Die physiologischen Funktionen der Haut werden durch ein Verbrennungstrauma geschädigt. Das Ausmaß der Schädigung ist abhängig von Temperatur, Einwirkdauer und -intensität sowie vom Aggregatzustand und der Art der Hitzequelle [65]. Wasser und Wasserdampf führen zu Verbrühungen. Offene Flammen, Wärmestrahlen, heiße Gase sowie feste und flüssige Körper verursachen Verbrennungen.

Ein Verbrennungstrauma der Haut kann, je nach Tiefenausdehnung der Schädigung, folgenfrei und ohne Funktionsverluste abheilen oder eine irreparable Schädigung hervorrufen. Die Schädigung einer Zelle durch Temperaturen von über $52^{\circ} \mathrm{C}$ führt zur Denaturierung der Proteine und zur Koagulationsnekrose. Durch die Zerstörung des Gefäßnetzes in der Endstrombahn werden Entzündungsmediatioren und toxische Eiweiße freigesetzt, die einen weiteren Entzündungsprozeß mit daraus resultierendem Zellschaden hervorrufen [48, 62].

Hautschädigungen durch Verbrennungen werden nach Jackson histologisch in drei Zonen eingeteilt (Abb. 2). 


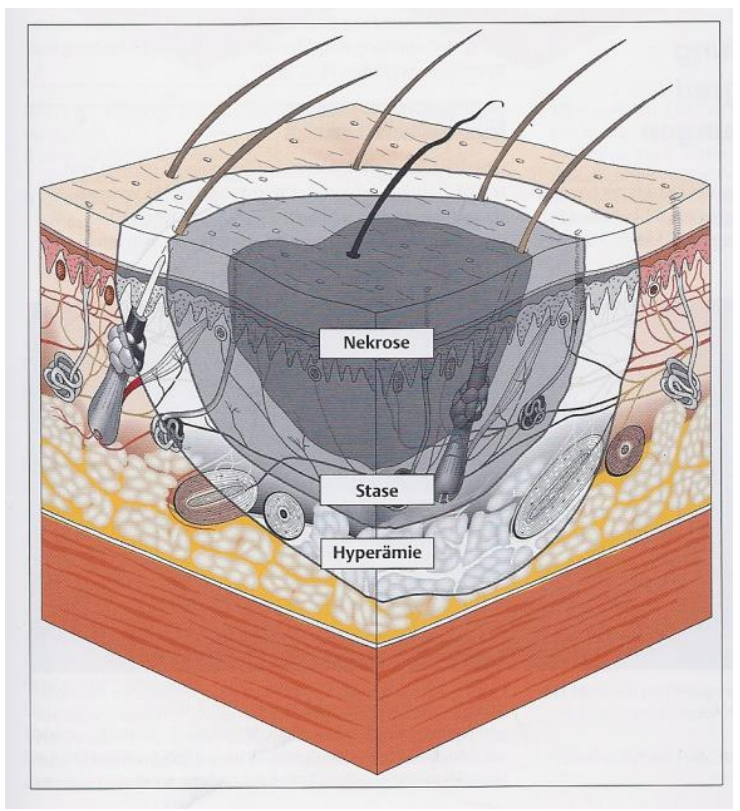

Abb. 2: Verbrennungszonen der Haut [28]. Abbildung aus [60]

Dabei wird eine konzentrische Hautschädigung beschrieben. Die zentrale Zone stellt die Region der intensivsten Hitzeaufnahme dar und wird als Koagulationsnekrose bezeichnet. In dieser Zone sind keine vitalen Zellen mehr vorhanden und das Gewebe ist somit irreparabel geschädigt. Die zentrale Zone wird von einer Zone der Stase umgeben, in der aufgrund der räumlichen Verflüchtigung der Hitzeenergie noch vitale Strukturen vorhanden sind. Die Gefäßzirkulation ist in diesem Bereich jedoch aufgrund vorhandener Vasokonstriktion vermindert. Daraus resultieren Mikrozirkulationsstörungen, die eine Ischämie verursachen können und somit zu einer sekundären Konversion der Stasezone in eine Koagulationsnekrose führen können. Die die Stasezone umgebende Schicht bildet die Zone der Hyperämie. Es liegt eine entzündungsbedingte Vasodilatation mit ausgeprägter Hautrötung vor. Diese Gewebezone heilt aufgrund der geringen Schädigung ohne Gewebeverlust vollständig ab.

\subsubsection{Einteilung der Verbrennungstiefe}

Wasser bzw. Wasserdampf verursacht häufig oberflächlichere Verbrennungen mit Blasenbildung. Flammen, Explosionen, Fett und heißes 
Metall rufen dagegen meist tiefere Gewebeschäden hervor. Dabei wird die Verbrennungstiefe in drei Schweregrade eingeteilt und nach klinischpathologischem Erscheinungsbild abgeschätzt. Die daraus resultierende Einstufung bestimmt das weitere Behandlungskonzept hat daher große prognostische Bedeutung für den Patienten.

\subsubsection{Verbrennungen ersten Grades}

Oberflächliche Verbrennungen werden als Verbrennungen ersten Grades (Abb. 3a) bezeichnet. Ausschließlich die Epidermis ist betroffen. Es liegt eine schmerzhafte Rötung vor. Die Haut ist dabei trocken und es besteht anfänglich ein brennender Schmerz, der später in Juckreiz übergeht. Innerhalb von wenigen Tagen wird die Epidermis durch eine vermehrte Proliferation der Keratinozyten narbenfrei ersetzt. Während des Regenerationsprozesses kommt es zu einer vermehrten Hautschuppung. Eine Verbrennung ersten Grades ist mit einem herkömmlichen Sonnenbrand vergleichbar [47].

\subsubsection{Verbrennungen zweiten Grades}

Bei Verbrennungen zweiten Grades reicht die Verbrennungstiefe bis in die Dermis hinein (Abb.3b). Zweitgradige Verbrennungen werden außerdem in oberflächlich dermale (Stadium 2a) und in tief dermale Läsionen (Stadium 2b) unterteilt. In beiden Fällen ist die Epidermis gänzlich zerstört. Bei der oberflächlich zweitgradigen Verbrennung erreicht die Schädigung aber nur die papillären Anteile der Dermis. Die dermalen Kapillaren werden verletzt, Plasma dringt in das Gewebe. Zusätzlich zu Hyperämie und Rötung zeigen sich daher Brandblasen [45]. Aufgrund der unmittelbaren Exposition der im papillären Anteil der Dermis vorhandenen Nervenenden ist die oberflächlich zweitgradige Verbrennung sehr schmerzhaft. Reagiert ein gerötetes Areal nach Druck mit Abblassen, rekapillarisiert aber rasch wieder, spricht dies für eine noch intakte dermale Durchblutung. Dieses Verhalten spricht klinisch für eine noch oberflächlich zweitgradige Läsion, die wegen der Proliferation und Migration von Keratinozyten, die in der tiefdermalen 
Schicht überlebt haben, innerhalb von zwei Wochen ohne ausgeprägte Narbenbildung abheilen kann [47].

Bei tief-dermalen zweitgradigen Verbrennungen wird die Dermis subtotal, aber bis in retikuläre Bereiche geschädigt. Aufgrund zerstörter Nervenendigungen ist das Schmerzempfinden vermindert. Die Verbrennungen erscheinen weiß und trocken, Blasen sind nicht mehr vorhanden. Auf Druck lässt sich kaum ein Kapillarfluss nachweisen. Die Fähigkeit zur Spontanheilung ist stark beeinträchtigt. Wunden dieser Art heilen stark verzögert unter ausgeprägte Narbenbildungstendenz ab. Eine operative Therapie ist somit indiziert [62].

\subsubsection{Verbrennungen dritten Grades}

Drittgradige Läsionen sind vollschichtige Verbrennungen und reichen von der Epidermis bis in die Subcutis (Abb. 3c). Intensiv verbrannte Haut erscheint verkohlt. Die Schmerzempfindung fehlt völlig. Der Wundgrund ist aufgrund der Denaturierung der gesamten Haut weiß, die Gefäße der Subcutis sind thrombosiert und schimmern dunkel durch den Verbrennungsschorf durch. Es besteht keine Möglichkeit einer Heilung und eine ausgedehnte operative Therapie ist erforderlich [47].

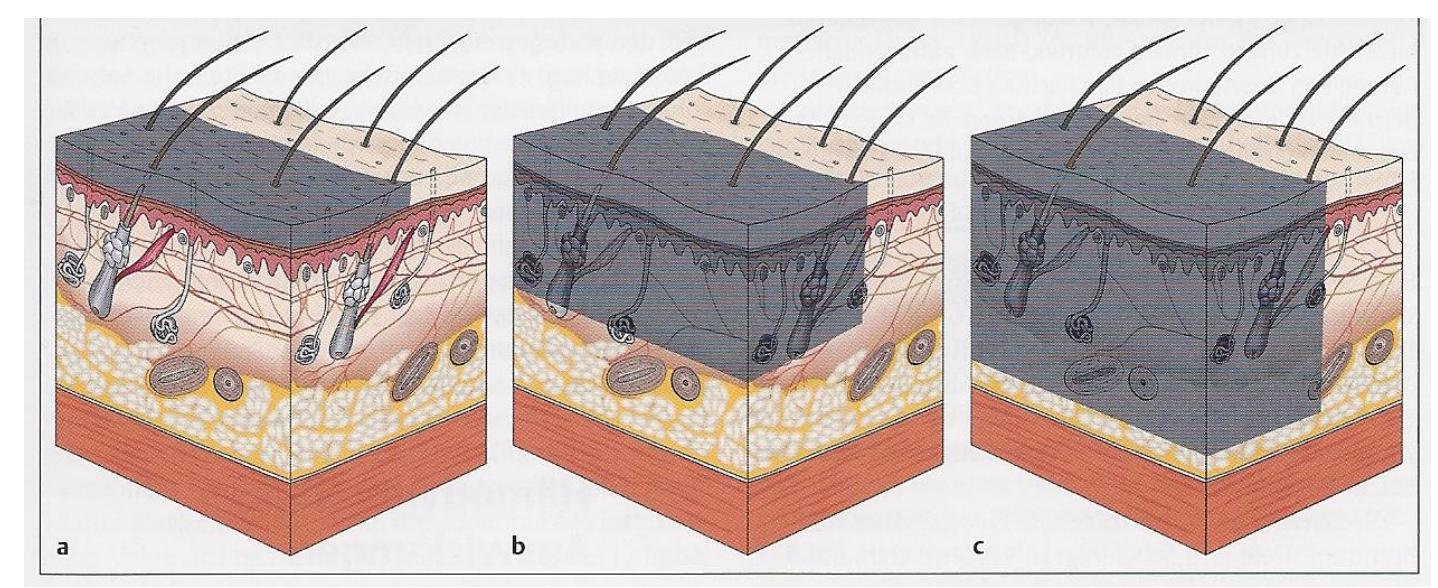

Abb. 3: Einteilung der Verbrennungstiefe (aus [60]) 
1.3.4. Beurteilung der Flächenausdehnung einer Verbrennungsverletzung

Für die Beurteilung der Größenordnung einer Verbrennungsverletzung der Haut ist die prozentuale Gesamtfläche der verbrannten Körperoberfläche (vKOF\%) zu bestimmen. Dafür ist die Neuner-Regel nach Wallace [30, 62, 63] gebräuchlich. Die Körperoberfläche eines Erwachsenen wird in einfach abgrenzbare Regionen aufgeteilt. Die besondere Praktikabilität dieser Methode ergibt sich daraus, dass elf derartige Regionen jeweils 9\% der Körperoberfläche entsprechen. Kopf und jede obere Extremität ergeben so jeweils 9\% KOF. Zweimal 9\% KOF entspricht der Rumpfvorder- und Rückseite. Ebenfalls 18\% KOF entspricht einer unteren Extremität. Das verbliebene 1\% KOF wird der Genitalregion zugeordnet (Abb. 4).

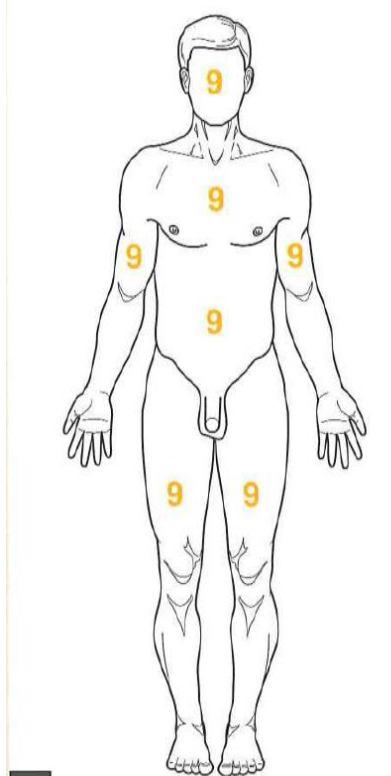

9a

Abb. 4: Die 9er-Regel (aus [26])

Eine im Alltag zur raschen Orientierung gebräuchliche Methode ist, die Größe der Handfläche der verletzten Person mit einem Prozent seiner Körperoberfläche gleichzusetzen (Abb. 5). Bei Kindern bestehen altersabhängig andere Körperproportionen, so dass die Neuner-Regel nicht angewendet werden darf [26]. 

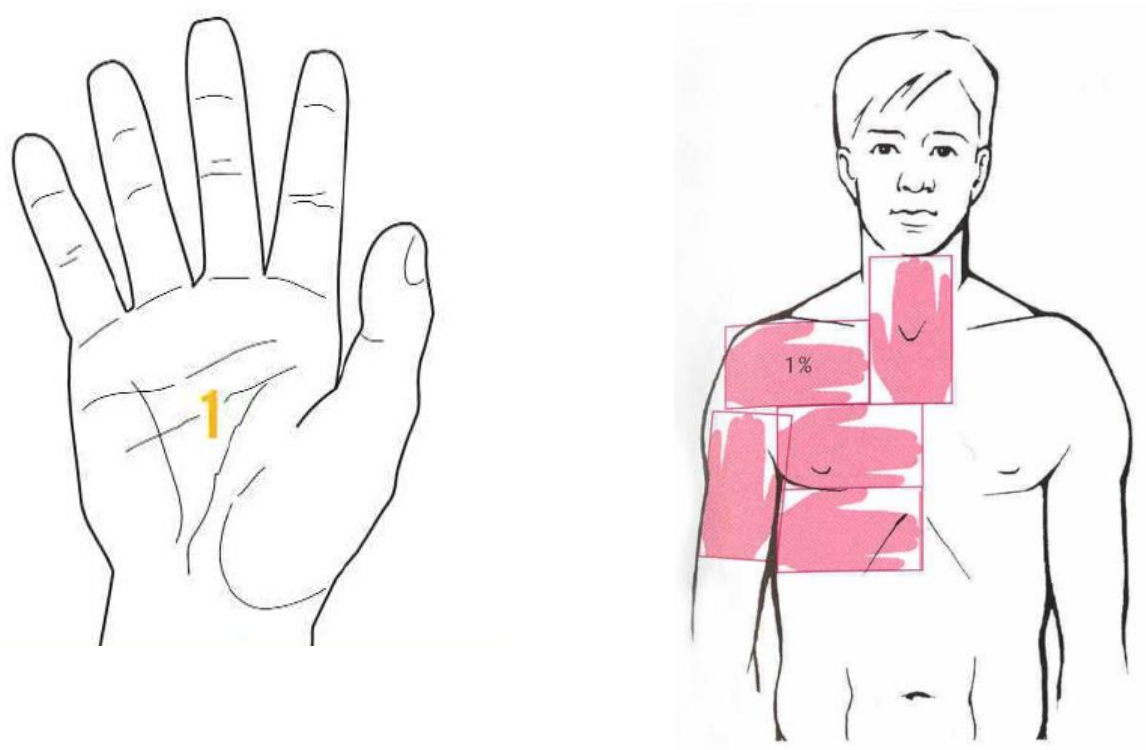

Abb. 5: Handflächenregel (aus [26])

\subsubsection{Therapie von Verbrennungswunden}

Die gründliche Reinigung der Verbrennungswunde ist Voraussetzung für eine gute Wundbehandlung. Erst danach ist die Beurteilung der Wundsituation möglich (Abb. 6 a-c).

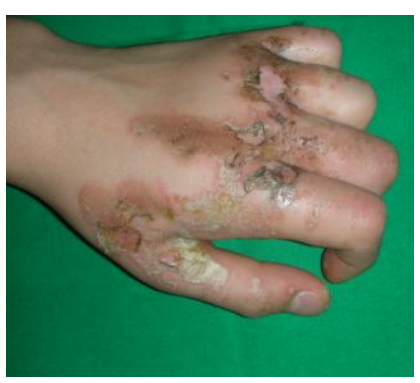

a

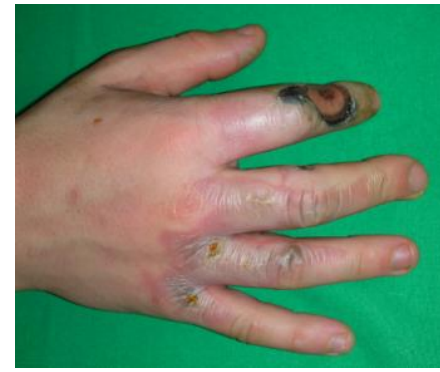

b

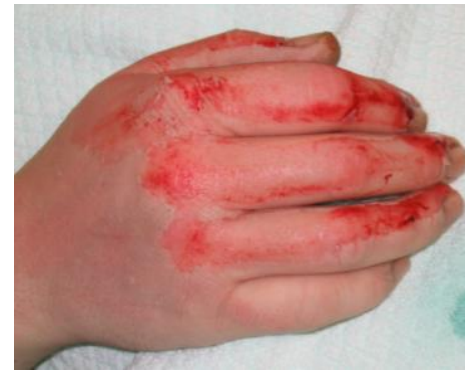

C

Abb. 6: Verschmutzte Verbrennungswunden an der Hand (a und b). Vor und nach Reinigung (b und $c$ )

Die Therapie der Verbrennungswunde richtet sich hauptsächlich nach deren Tiefenausdehnung. Erstgradige Läsionen gleichen einem Sonnenbrand und erfordern keine spezielle Therapie. Kühlende Umschläge oder Gele sind meist ausreichend. Bei Verbrennungen zweiten Grades, die ausschließlich die oberflächlichen Dermisanteile betreffen, erfolgt die 
Therapie ebenfalls konservativ. Man verwendet antiseptische Lösungen und Fettgazeverbände. Das Ziel ist die Vermeidung von Infektionen an den nach erfolgter Blasenabtragung deepithelisiert exponierten und zunächst sterilen Hautarealen [45]. Durch temporäre alloplastische Dermisersatzmaterialen (Abb. 7) wird eine Keimbarriere gebildet und ein Flüssigkeitsverlust verhindert, was die Wundheilung fördert. Schmerzhafte Verbandswechsel müssen seltener erfolgen.

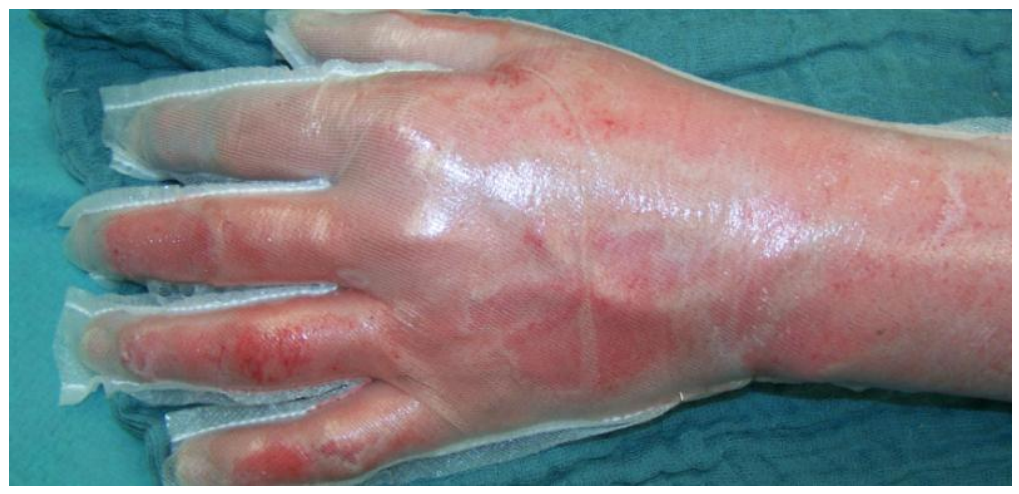

Abb. 7: Versorgung einer oberflächlich zweitgradigen Verbrennungs an der Hand mit einem temporären Ersatzmaterial (Biobrane ${ }^{\circledR}$ ) [6]

Während oberflächlich zweitgradige Verbrennungen konservativ behandelt werden, erfolgt bei tiefdermalen zweit- und drittgradigen Verbrennungen eine operative Therapie. Die Bestimmung der Verbrennungstiefe ist somit von essentieller Bedeutung. Da die Schädigungstiefe auch Stunden nach der eigentlichen Schädigung noch zunehmen kann („Nachtiefen“), soll sie in dieser Zeit mehrfach überprüft werden (Abb. 8).

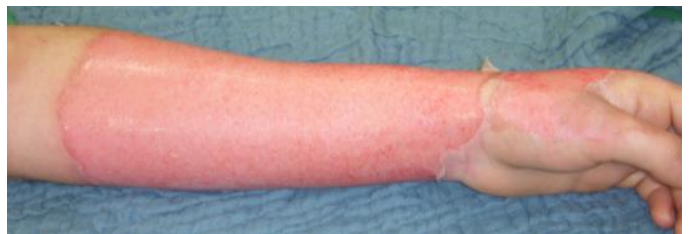

a

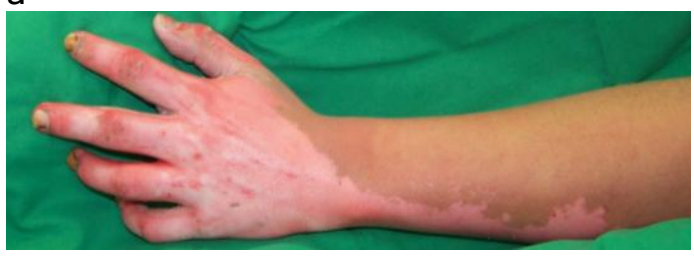

C

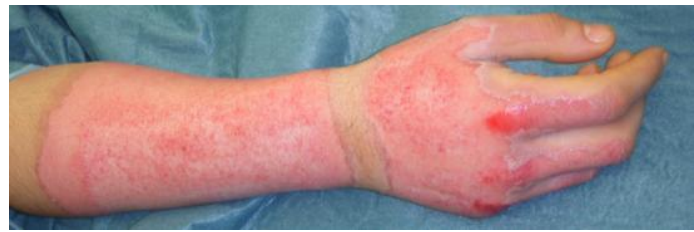

b

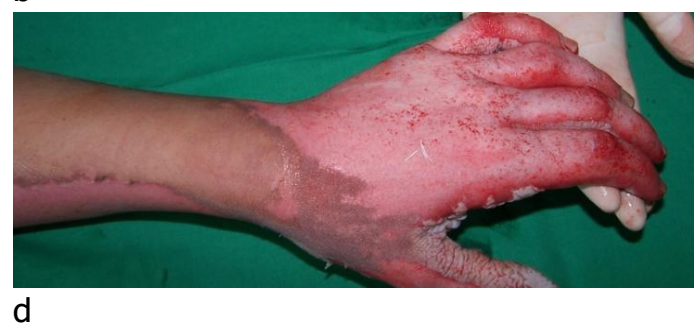

Abb. 8: Unterschiedlicher Schädigungsgrad bei Handverbrennung. a und b: oberflächlich zweitgradig (2a); c und d: tief zweitgradig (2b) 
Operationspflichtige Verbrennungswunden sollten frühzeitig, möglichst innerhalb der ersten 48 Stunden, nekrosektomiert werden (Abb. 9). Eine frühzeitige Entfernung des nekrotischen Gewebes kann septische Komplikationen verhindern und so die Überlebenswahrscheinlichkeit des Schwerbrandverletzen erhöhen [43]. Auch funktionelle und ästhetische Ergebnisse werden durch eine frühzeitige operative Therapie verbessert [24].

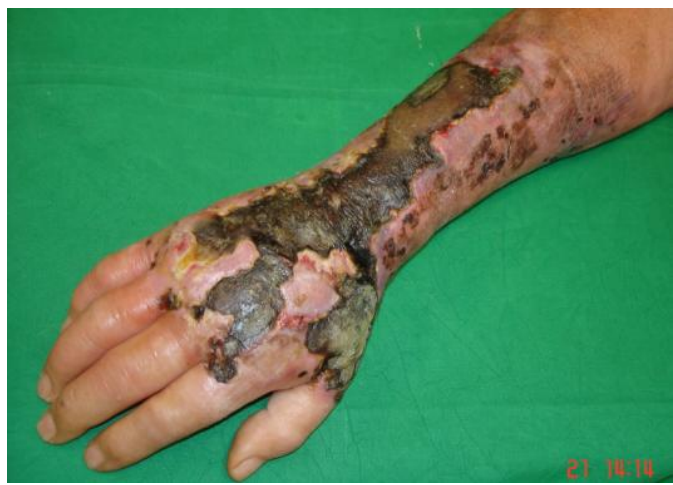

a

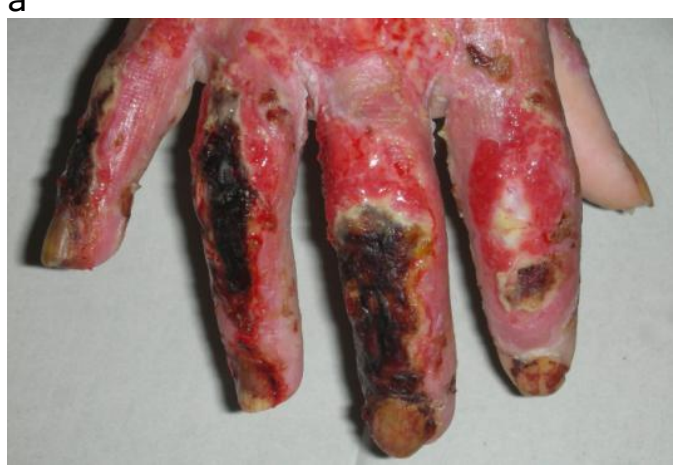

C

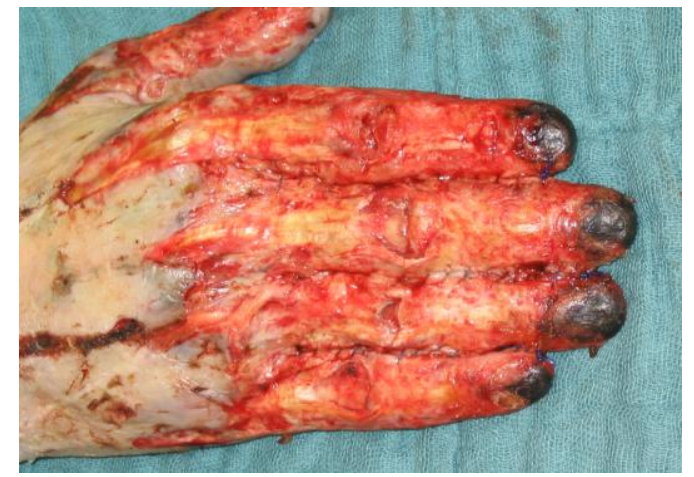

b

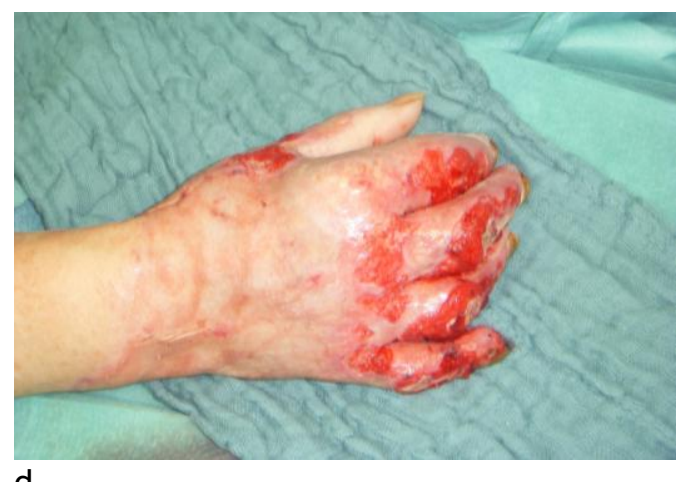

d

Abb. 9: Drittgradige Verbrennungen der Hand vor (a-c) und nach Nekrosenabtragung (d)

Zwei chirurgische Standardverfahren stehen bei der Nekrosektomie zur Auswahl. Bei erhaltenem subdermalen Gefäßplexus erfolgt eine tangentiale Nekrosektomie (Abb.10). Nur wenn keine durchbluteten Dermisanteile mehr vorhanden sind, erfolgt eine epifasziale Nekrosektomie, bei der die gesamte Dermis zusammen mit der Subcutis bis auf die darunterliegende Faszie entfernt wird (Abb. 11). Mit Dermatom (Abb. 12 d) werden Epidermis und Dermis schichtweise abgetragen, bis ein gut durchbluteter Wundgrund sichtbar wird (Abb. 10b und 12e). Auf diesen Wundgrund kann anschließend die Defektdeckung mit autologer Spalthaut erfolgen. Diese heilt sowohl auf gut durchbluteter Dermis als auch auf Muskelfazie ein und 
kann so den Defekt zuverlässig und sicher geschützt vor Flüssigkeitsverlust, Austrocknung und Keimbesiedlung verschließen. Die Hauttransplantate werden in einer Dicke von 0,2 - 0,3 mm von unverbrannten Körperregionen entnommen. Die Entnahmestellen heilen in der Regel spontan. Dünne Spalthauttransplantate heilen schneller ein, können aber zu stärkeren Narbenkontrakturen führen als dickere Transplantate. Durch maschenförmiges Schneiden der gewonnenen Haut („meshen“) kann das Transplantat eine größere Wundfläche als das Entnahmeareal bedecken [45]. Besonders bei großflächigen Verletzungen mit entsprechend begrenzten Spenderbezirken kann durch dieses Verfahren noch eine Deckung mit autologer Haut erreicht werden. Das typische gitterartige Muster bleibt nach dem Einheilen allerdings sichtbar und stellt daher einen ästhetischen Makel dar. Daher sollen Spalthauttransplantate an den Händen nicht gemesht, sondern nur skarifiziert werden. Über kleine Stichinzisionen kann Wundsekret abfließen und ein Abheben der Spalthaut vom Untergrund kann verhindert werden (Abb. $12 \mathrm{f} ; 13 \mathrm{c})$.

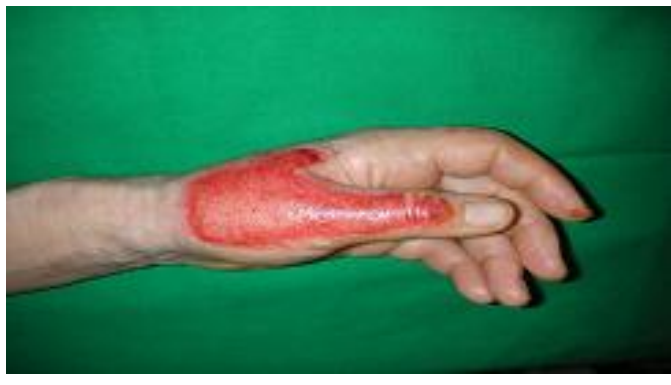

a

Abb. 10 a und $\mathbf{b}$ : tangentiale Nekrosektomie bei oberflächlich zweitgradiger Verbrennung der Hand

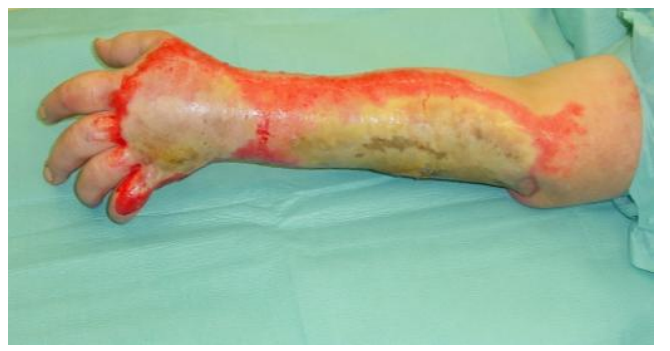

a

Abb. 11 a und b: epifasziale Nekrosektomie bei tief dermaler Verbrennung an Hand und Unterarm

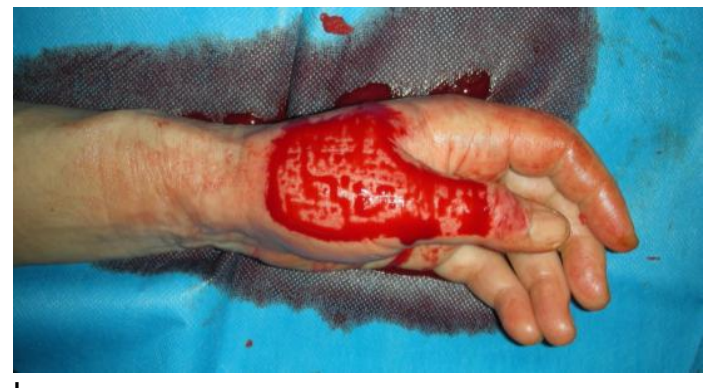

b

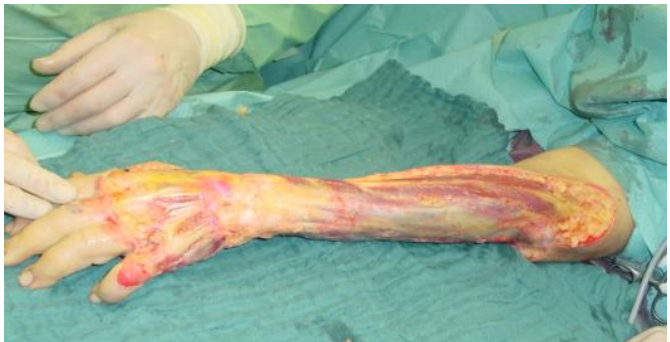

b 

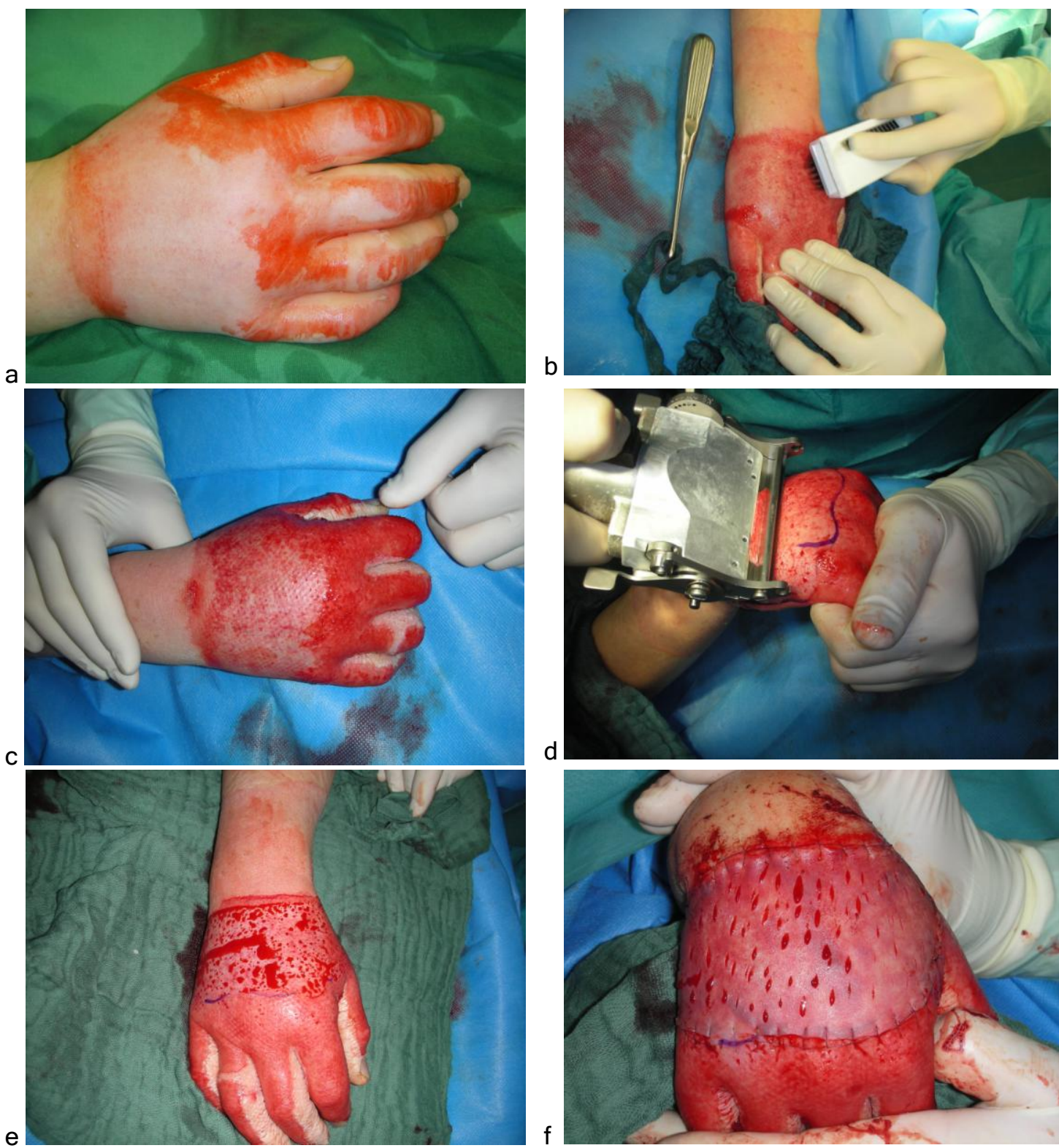

Abb. 12: Tief zweitgradige Verbrennung am Handrücken (a); Wundreinigung (b); Kontrolle der Verbrennungstiefe (c); tangentiale Nekrosektomie mit dem Dermatom (d); Wundgrund nach Nekrosektomie (e); Defektdeckung mit skarifizierter Spalthaut (f)

Innerhalb von drei bis fünf Tagen sprießen Kapillaren in die transplantierten Areale ein und sorgen für die Ernährung der Haut, die bis dahin nur über Diffusion erfolgen kann [22]. Damit diese Kapillaren nicht durch Scherkräfte abreißen, müssen die an den Rändern mit Nähten fixierten Transplantate zusätzlich durch sorgfältige Wundverbände mit Fettgaze sowie Polsterung und Kompression gesichert werden (Abb.13 c-d). 

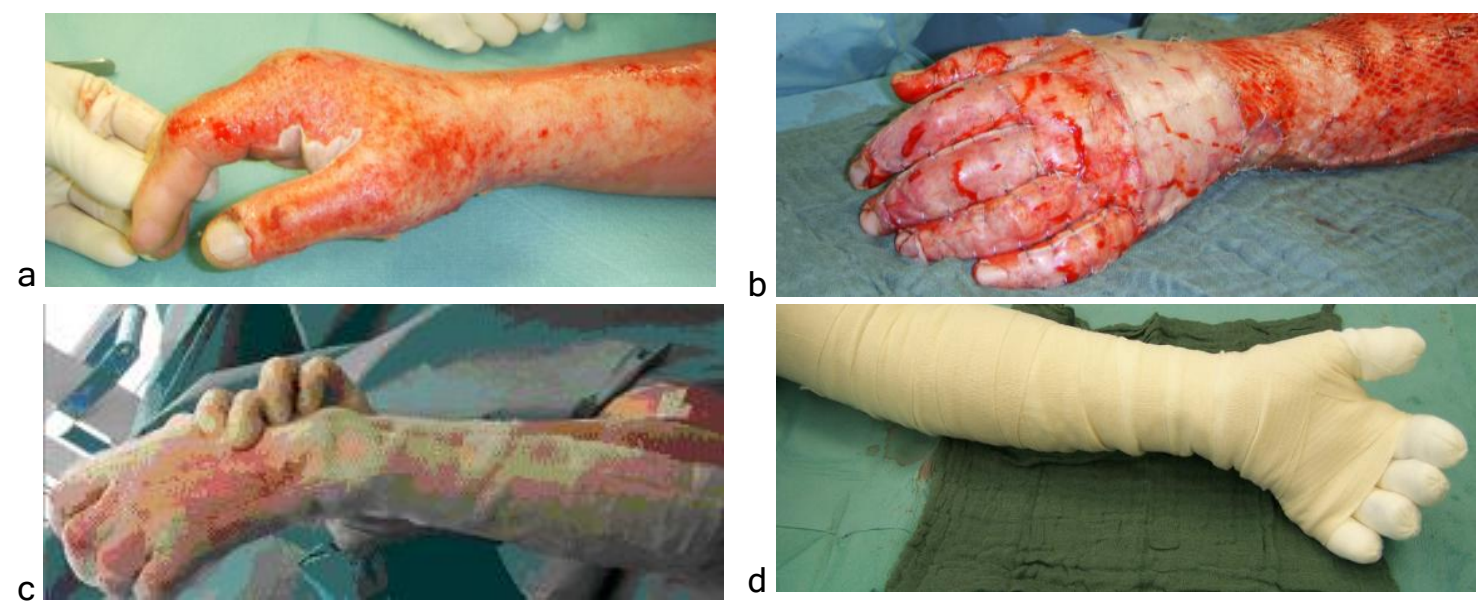

Abb. 13: Tief zweitgradige Verbrennung an der Hand (a); nach Nekrosektomie und Spalthauttransplantation (b); steriler Wundverband mit Fettgaze (c); Druckverband zur Verbesserung der Transplantateinheilung (d)

\subsubsection{Thermisch geschädigte Hände}

Über $80 \%$ aller Brandverletzten haben Verbrennungen an den Händen [39]. Obwohl die Hand einen nur geringen Anteil an der gesamten Körperoberfläche einnimmt, werden Verbrennungen an der Hand als schwere Verletzung eingestuft (ABA). Funktionelle Defizite und Einschränkungen der Lebensqualität können resultieren [50, 54].

Die Haut der Hände zeichnet sich durch einige anatomische Besonderheiten aus. Sie besitzt eine besonders hohe Kapillardichte im Stratum papillare, ihre sensorische Qualität und ihre physikalische Belastbarkeit sind auf der Innenseite besonders stark ausgeprägt. Dabei weist die Haut des Handrückens viele Unterschiede zur Haut der Handfläche auf. Am Handrücken ist die Haut dünner und leichter verschiebbar. Die Beugung der Fingergelenke wird dadurch begünstigt. Funktionell wichtige Strukturen wie Gelenke und Sehnen liegen dicht unter der Hautoberfläche. Die Haut an der Handfläche ist fest mit der Palmaraponeurose verwachsen und dadurch druckresistenter [49]. Die Handfläche enthält eine hohe Dichte an sensorischen Endorganen, so dass Verbrennungen in diesem Bereich $\mathrm{zu}$ erheblichen sensorischen Einschränkungen führen. Gleichzeitig ist die Haut an der Handfläche dicker als am Handrücken und toleriert somit höhere Temperaturen. Zum Schutz vor Flammen werden die Hände typischerweise vor das Gesicht gehalten, 
wodurch der Handrücken häufiger als die Handfläche von Verbrennungen betroffen ist [52]. Demgegenüber treten bei kleinen Kindern tiefgradige Verbrennungen der Handfläche auf, wenn sie beginnen, Gegenstände zu ergreifen oder zu berühren.

Das Behandlungskonzept bei einer Verbrennungsverletzung der Hand folgt der jeweiligen Verbrennungstiefe. Dabei ist die frühfunktionelle Wiederherstellung der Handfunktion richtungweisend. Oberflächliche Verbrennungen der Hände heilen innerhalb von zwei Wochen spontan ab und bedürfen keiner chirurgischen Intervention. Eine tägliche Wundpflege mit modernen Wundauflagen führt zu guten funktionellen Ergebnissen. Ab einer tief zweitgradigen Verbrennung (2b) ist eine Restitutio ad integrum nicht mehr möglich und eine operative Therapie erforderlich. Nekrosen werden abgetragen und die betreffenden Areale mit Hauttransplantaten gedeckt. Dabei ist die unterschiedliche Beschaffenheit der Haut an Beugeund Streckseite der Hand zu berücksichtigen. Die Dicke der transplantierten Haut sollte an die Belastungszonen des jeweiligen Areals der Hand angepasst werden. Überlässt man Wunden der sekundären Abheilung und wartet die Epithelisation von Granulationsgewebe ab, wird das Integument sich dauerhaft nachteilig verändern. Auch nach Defektdeckung durch transplantierte Spalthaut entstehen narbige Veränderungen. Durch langwierige Umbauprozesse verändert sich die Hautnarbe (Maturation), überschießende Narbenbildung treten ebenso wie Schrumpfungen auf. Besonders im Verlauf der randbildenden Narben an der Grenze zwischen Transplantaten und gesundem Gewebe entstehen Kontrakturen, häufig in den Zwischenfingerfalten, die dann die Beweglichkeit behindern (Abb. 14 ae). Narben führen außerdem zu Textur- und Pigmentveränderungen (Abb. 15 a-b; 16 a-d; 17 a-b). 

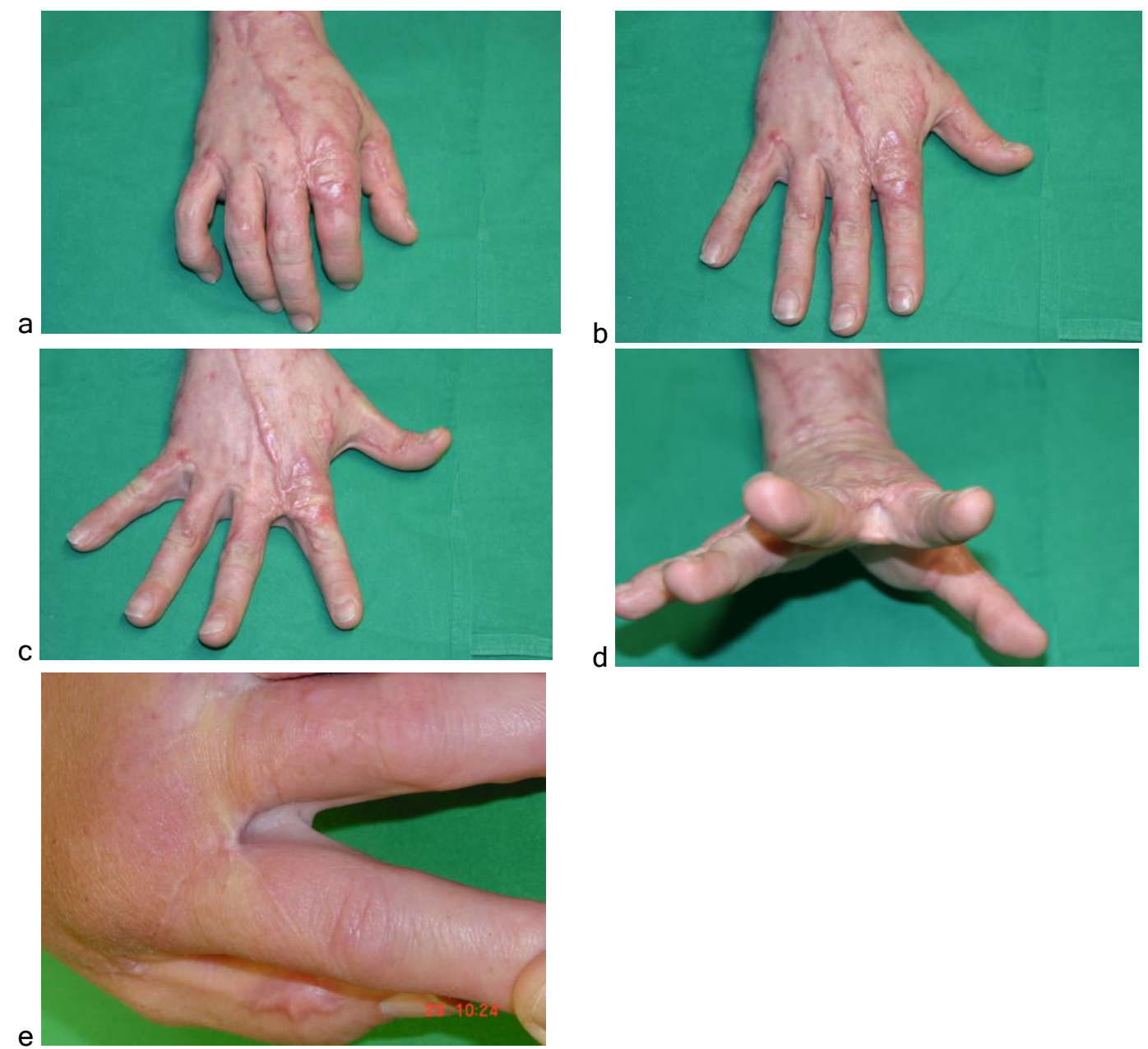

Abb. 14: Hypertrophe Narben mit Kontrakturen und narbiges Pterygium in der zweiten Kommissur nach Verbrennung der Hand

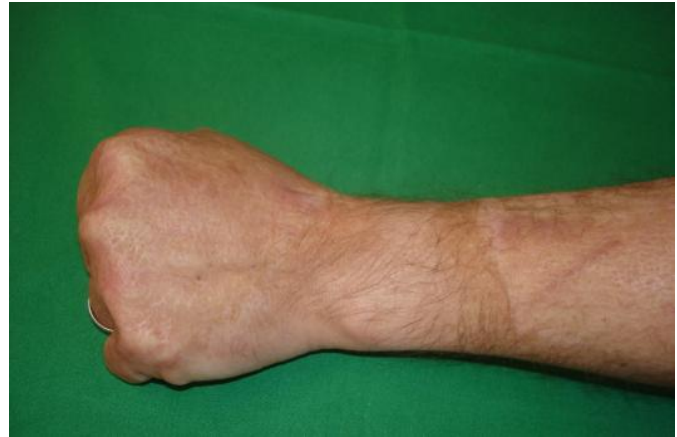

a

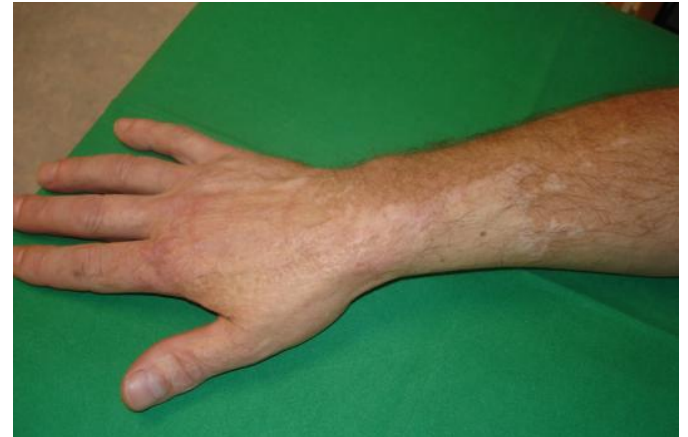

b

Abb. 15 a und b: Hypopigmentation mit ansonsten günstigem funktionellem und ästhetischem Ergebnis nach Spalthauttransplantation bei Verbrennungsverletzung an Hand und Unterarm 

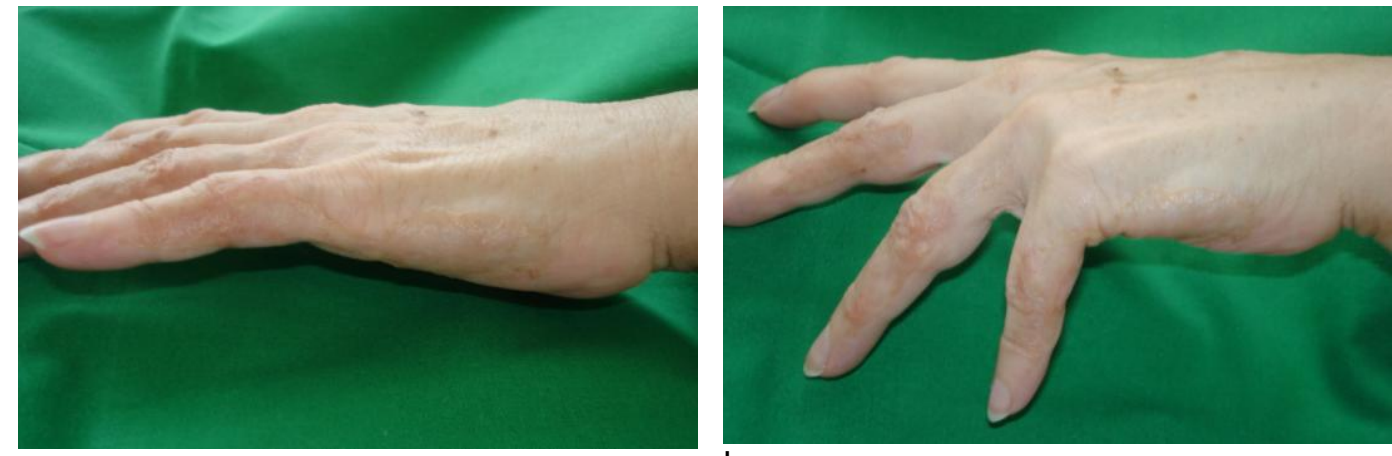

a

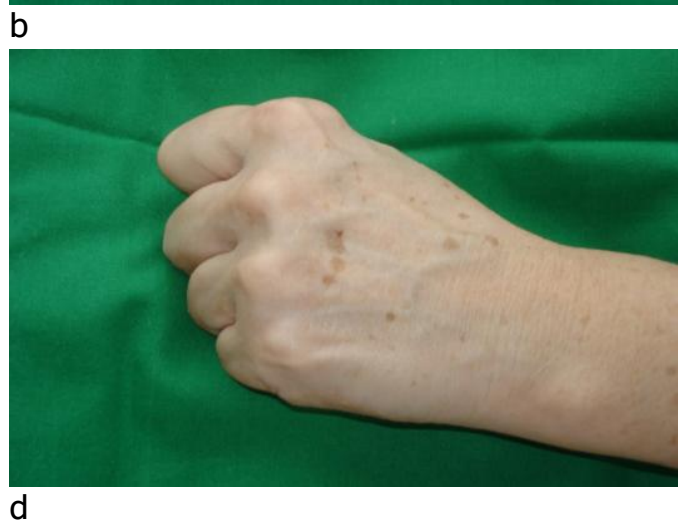

C

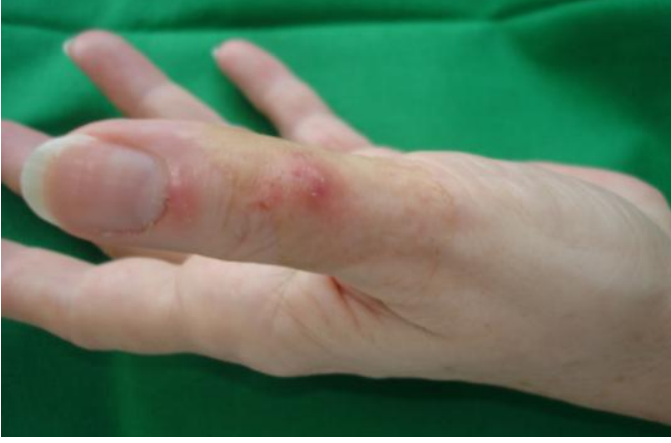

d

Abb. 16 a-d: Hyperpigmentation nach Spalthauttransplantation bei Handverbrennung

$\mathrm{Da}$ die Handfläche einer besonders starken mechanischen Belastung ausgesetzt ist, bleibt diese Region trotz abgeschlossener Wundheilung oftmals aufgrund mangelnder Stabilität vulnerabel (Abb. 18 a-c). Das Beschwerdebild wird jedoch nicht nur durch die daraus resultierenden funktionellen Einschränkungen geprägt, sondern beinhaltet zusätzlich vegetative und somatische Komponenten. Die Temperaturregulation ist beeinträchtigt, es besteht eine erhöhte Empfindlichkeit für Kälte und/oder Wärme. Wegen Störungen der Sensibilität werden oft Taubheitsgefühle angegeben. Die Gleitfähigkeit der Muskeln und Sehnen ist vermindert, so dass Gelenk- und Gliederschmerzen resultieren. Wenn wichtige Hautanhangsgebilde wie Talg- und Schweißdrüsen verloren gegangen sind, weist die Haut eine erhöhte Trockenheit und Instabilität auf, die mit erhöhter Verletzlichkeit, Spannungsgefühl und Juckreiz einhergehen [16, 27]. 

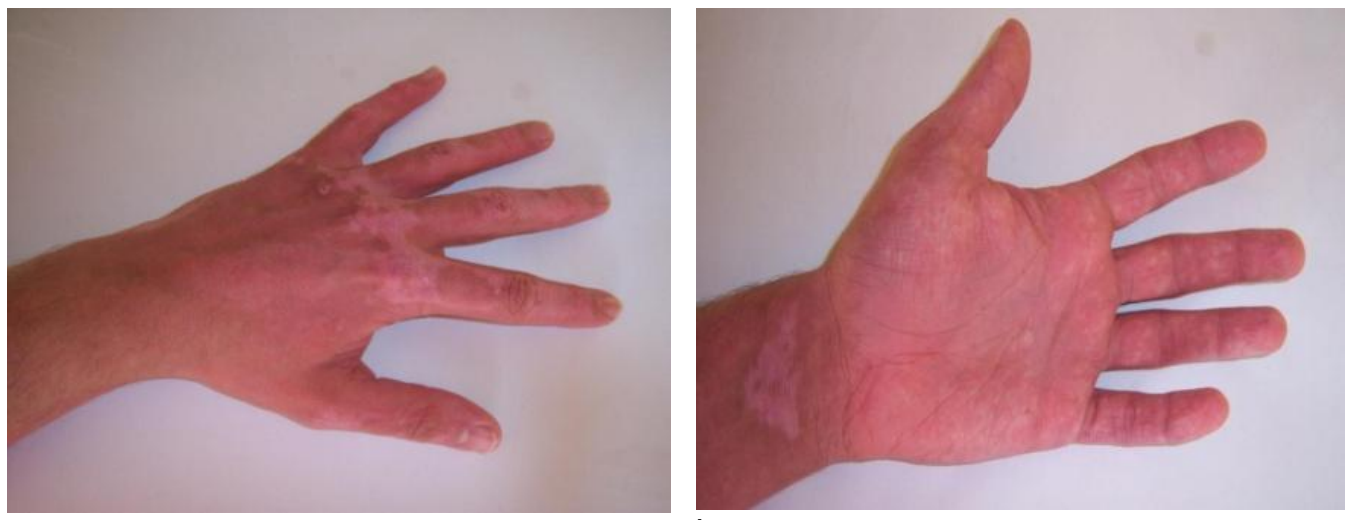

a

b

Abb. 17: Hypopigmentation nach konservativ behandelter oberflächlich zweitgradiger Verbrennung
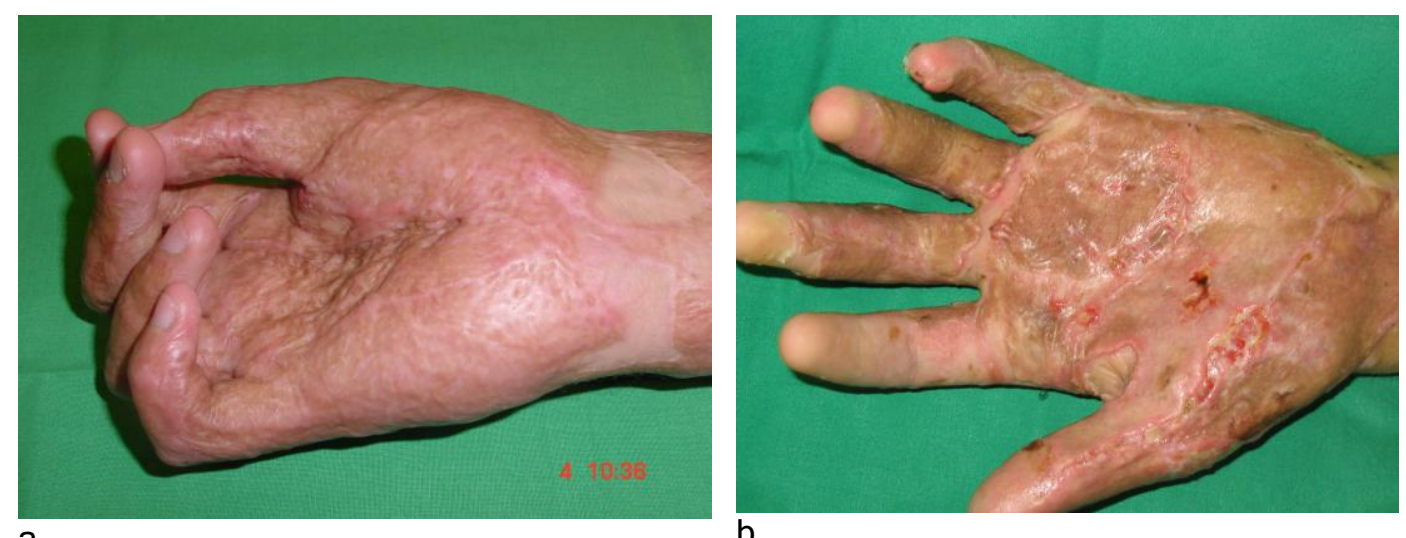

a

b

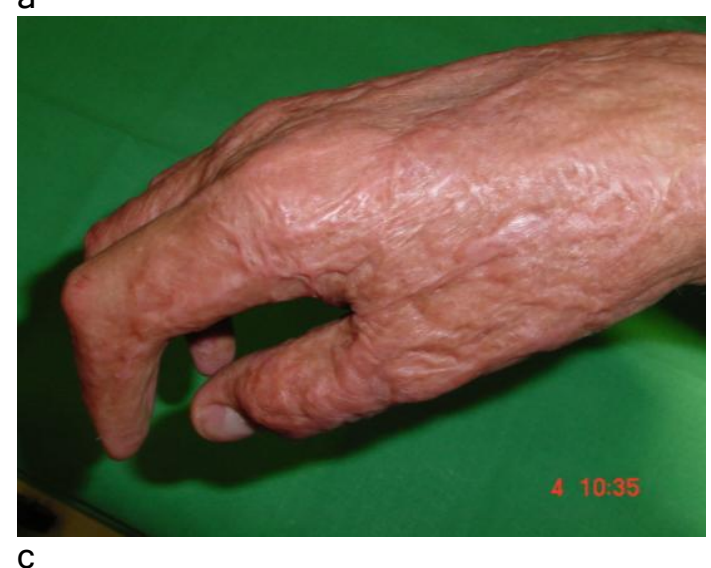

Abb. 18 a-c: Hypertrophe Narbenbildung nach konservativ abgeheilter tief dermaler Verbrennunq der Hand. Ausgeprägte Kontrakturstränge in der Hohlhand (a), vereinzelte instabile ulzerierte Areale (b). Stigmatisierender Handrücken mit sichtbaren Kontrakturen (c).

Handverbrennungen haben zwar kaum Einfluss auf die Überlebensrate der Betroffenen, dennoch sind ihre Folgen nach erfolgter Rehabilitation der wichtigste prognostische Faktor für einen erfolgreichen Wiedereintritt in Gesellschaft und Berufsleben [29]. Neben der frühzeitigen Beübung der 
Finger, um Einsteifungen und Funktionsverluste zu verhindern, ist das äußere Erscheinungsbild der Hand nach abgeschlossener Wundheilung wichtig. Ein zufriedenstellendes Behandlungsergebnis setzt eine hohe Versorgungsqualität und optimale interdisziplinäre Therapiekonzepte von Plastischen Chirurgen, Ergo- und Physiotherapeuten, Psychologen und Pflegekräften voraus [13].

\subsubsection{Lebensqualität}

Narbenbildungen an den Händen können auch ohne funktionelle Beeinträchtigung zur Berufsunfähigkeit führen. Das Körperbild ist ein elementarer Teil unserer Identität. Der Brandverletzte ist sichtbar stigmatisiert. Sind die Hände betroffen, lassen sich entsprechende Verletzungsfolgen im Alltag kaum verbergen. Sowohl die kosmetische Deformität als auch die funktionelle Behinderung durch Gelenkkontrakturen können schwerwiegende physische und psychische Auswirkungen auf den Brandverletzten haben. Narbig abgeheilte Areale bedingen somit sowohl funktionell wie auch ästhetisch eine Minderung der Lebensqualität.

Lebensqualität lässt sich nicht ohne weiteres klar definieren, denn sie ist multidimensional. Sie setzt sich aus dem funktionalen Status der somatischen Ebene, der psychischen und der sozialen Dimension zusammen [37]. Dabei werden Aspekte wie körperliche Beschwerden, Schmerz, emotionales Erleben, Anpassungsmöglichkeiten, soziale Kontakte, die Fähigkeit zur Beziehungsherstellung, Arbeit und Leistung, die finanzielle Situation, Religiosität und ethisch motiviertes Wertverhalten integriert. Wird die Lebensqualität auf die gesundheitsbezogene Ebene reduziert, so dass gesundheitsunabhängige Aspekte wie Einkommen, Religion und Umwelteinflüsse wegfallen, kann sie als "persönliche Einschätzung des eigenen körperlichen und psychischen Befindens und der sozialen Integration nach Einbeziehung des Einflusses von Krankheit und Behandlung" definiert werden [32]. Innerhalb dieser Dimension kann zwischen der allgemeinen, d.h. gesundheitsbezogenen, und der krankheitsspezifischen Lebensqualität unterschieden werden. Gängige 
Messinstrumente der Lebensqualität sind standardisierte Fragebögen. Bei der krankheitsspezifischen Lebensqualität erfolgt dabei die spezifische Erfassung von Belastungen, die nur für Betroffene mit der jeweiligen Erkrankung gelten. Die gesundheitsbezogene Lebensqualität wird wiederum durch Fragen aus dem Alltagsleben erfasst.

Bei dauerhaften körperlichen Funktionsbeeinträchtigungen nach Verbrennungsverletzungen, die unter Umständen Jahre zurückliegen, besteht eine hohe Korrelation zu depressiven Störungen, und zwar durchaus nicht nur für schwerwiegende Einschränkungen. Ähnliches gilt auch für leichtere funktionelle Defizite. Weniger stark korreliert der Grad depressiver Störungen mit dem Flächenausmaß der ursprünglichen Verbrennungsverletzung [44]. 


\section{Fragestellung}

Aus dem zuvor Dargestellten ergibt sich, dass Verbrennungsverletzungen besonders an den Händen zu schwerwiegenden Folgeschäden führen. Zwar ist für das Endergebnis in funktioneller, ästhetischer und auch psychosozialer Hinsicht vor allem eine optimale Primärbehandlung mit nachfolgender Rehabilitation verantwortlich. Gleichwohl dürfen die erst später eintretenden Effekte wie die nachfolgende Narbenmaturation nicht unterschätzt werden. Ebenso ist es von großer Bedeutung, etwaige Besonderheiten beruflicher oder sonstiger Exposition zu erkennen, die zu sekundären Problemen an eigentlich bereits abgeheilten Händen führen. Allgemein lässt sich sagen, dass Verbrennungspatienten häufig jahrelang behandlungsbedürftig sind, bis ein tragbarer Endzustand von Form und Funktion erreicht wird [2]. Regelmäßige Nachuntersuchungen sind daher von großer Bedeutung.

Dies ist aus unterschiedlichen Gründen im klinischen Alltag allerdings oftmals nicht stringent durchführbar. Zur Erfassung des Folgezustands ist daher ein Fragebogen, den der Patient daheim bearbeiten kann, ein möglicher Ausweg.

Ein derartiges Instrument ist der seit langem etablierte DASH-Fragebogen, der nicht nur individuelle Aussagen erlaubt, sondern auch in vergleichenden klinischen Studien regelmäßig Verwendung findet. Auch bei der gutachterlichen Einschätzung von Verletzungsfolgen ist dieser Fragebogen hilfreich. Allerdings folgt er dem Anspruch, gleichsam alle denkbaren Folgen von Verletzungen der oberen Extremität zu erfassen. Die dargestellten Besonderheiten der Verbrennungsfolgen an der Hand lassen sich mit dem DASH-Fragebogen also nicht adäquat abbilden.

Aus diesem Grund haben wir die Frage verfolgt, ob sich ein erweiterter Fragenkatalog definieren lässt, der im Sinne eines neuen Fragebogens die Folgen nach Brandverletzungen an Händen besser erfassen kann. Idealerweise soll ein derartiger Fragebogen zur Erkennung von behandlungsfähigen Verletzungsfolgen beitragen, aber auch in klinischen 
Studien als Instrument zur vergleichenden Betrachtung verschiedener Behandlungsmethoden verwendet werden und schließlich auch zur Bestimmung des Maßes von Verbrennungsfolgen im Entschädigungsrecht geeignet sein. 


\section{Patienten und Methodik}

\subsection{Einschlusskriterien}

In die Studie wurden alle Patienten aufgenommen, die zwischen dem 1.1.2006 und dem 31.12.2007 im Zentrum für Schwerbrandverletzte des Klinikums Nürnberg wegen einer Brandverletzung stationär behandelt wurden, sofern sie auch Verbrennungen an der Hand erlitten hatten, die oberflächlich zweitgradig oder tiefer waren. Von insgesamt 211 in diesem Zeitraum behandelten Patienten konnten 81 in der Studie erfasst werden.

\subsection{Datenerfassung}

3.2.1. Retrospektive Analyse der Falldokumentation

Aus den Behandlungsunterlagen wurden die folgenden Informationen entnommen und erfasst:

- Alter des Patienten zum Zeitpunkt der Verletzung

- Geschlecht des Patienten

- Unfallursache

unterschieden nach

- Verletzung durch offene Flammen

- Unfall in geschlossenem Raum

- Unfall im Freien

- Kleidungsbrand

- Fahrzeugbrand

- Grillunfall

- Explosion

- Verpuffung

- Stromunfall

- Lichtbogenverletzung

- Starkstromverletzung

- Blitzschlag

- Verbrühungen durch heisse Flüssigkeiten

- Brennende Flüssigkeiten 
- Kontakt an heissen Oberflächen

- Massive UV-Bestrahlung

- Autoaggressive Handlung

- Privater Unfall / Arbeitsunfall

- Dauer der Behandlung auf der Intensivstation

- Dauer der anschließenden Behandlung auf der Normalstation

- Flächenausdehnung der Brandverletzung

- Tiefenausdehnung der Brandverletzung

- Anzahl der erforderlichen Operationen

- Anzahl der Operationen ausschließlich an den Händen

Diese Daten wurden elektronisch in Tabellenform erfaßt und numerisch umkodiert, so dass deskriptive Statistiken berechnet werden konnten.

Die 81 teilnehmenden ehemaligen Patienten erhielten zwei Fragebögen, den DASH - Bogen (Anlage 1) und den von uns entwickelten Bogen zum „Nürnberger Fragebogen Handverbrennung“ (NFH, Anlage 2).

\subsubsection{DASH - Fragebogen}

Der "Disabilities of arms, shoulder and hand questionaire" wurde ursprünglich 1996 von der "American Academy of Orthopedic Surgeons" gemeinsam mit dem "Council of Musculoskeletal Specialty Societies" und dem "Institute for Work and Health" der Universität Toronto (Kanada) entwickelt.

Mit diesem Fragebogen lassen sich Funktionseinschränkungen der gesamten oberen Extremität erfassen. Er richtet sich also an Menschen, die zuvor eine Verletzung oder andersartige Schädigung an der oberen Extremität erlitten haben. Der Patient äußert sich zu seinen Beschwerden und beantwortet Fragen zu seinen Alltagsaktivitäten und Routineaktivitäten, wie er in seinem beruflichen, sozialen und privaten Umfeld zurechtkommt.

Der Fragebogen gliedert sich in ein Hauptmodul sowie optionale Ergänzungselemente. In unserer Studie haben wir den Fragebogen in 
seiner 1999 publizierten Fassung [18] verwendet und in der dort beschriebenen Weise ausgewertet (Anlage 1).

Im ersten Abschnitt (Teil A) werden 23 Fragen zu verschiedenen Aktivitäten und Tätigkeiten gestellt. Der dann folgende Teil B besteht aus weiteren sieben Fragen, in denen nach verschiedenen Beschwerdeangaben gefragt wird. Optional können in Teil C Musiker und Sportler spezielle Angaben zu ihrer besonderen Situation machen.

Der DASH-Score ist geeignet, Schädigungsfolgen zu quantifizieren und kann als Instrument zur Bewertung des Therapieverlaufs und zur Behandlungsplanung eingesetzt werden. $\mathrm{Er}$ hat sich für veschiedenste klinische Entitäten bewährt und ist daher international anerkannt und gebräuchlich. Insbesondere wird er auch zur Evaluation von thermischen Schäden angewendet $[6,23,40]$.

\subsubsection{Nürnberger Fragebogen Handverbrennung}

Die vom Nürnberger Fragebogen verwendeten Parameter sind speziell auf die Folgen von Verbrennungsverletzungen an der Hand abgestellt. Er stellt daher nicht nur eine Weiterentwicklung des DASH-Fragebogens dar. Vielmehr verzichtet er darauf, dessen Fragen zu wiederholen.

Bei der Zusammenstellung der abgefragten Aspekte wurde auch auf das seit 1995 gebräuchliche Schema zur Bestimmung der Minderung der Erwerbsfähigkeit nach Brandverletzungen zurückgegriffen [5, 25]. Die Parameter, für die dies zutrifft, sind in der folgenden Auflistung der im Nürnberger Fragebogen enthaltenen Items mit einem Stern $\left(^{*}\right)$ gekennzeichnet.

- Alter des Patienten Unfallzeitpunkt und zum Zeitpunkt der Befragung

- Beschäftigung noch immer im ursprünglichen Beruf

- Auswirkung der Handverbrennung auf die berufliche Tätigkeit

- Bewertung des durch die Operation/Behandlung erzielten Ergebnisses

- Bewertung des Aussehens der Hände 
- Werden die Hände in der Öffentlichkeit versteckt?

- Werden die Hände zum Gestikulieren mehr oder weniger verwendet?

- Farbunterschiede*

- Sensibilität

- Schmerzen (verbal - numerisch - visuell)

- Kälteempfindlichkeit ${ }^{*}$

- Wärmeempfindlichkeit ${ }^{\star}$

- Trockenheit der Haut ${ }^{\star}$

- Verletzlichkeit der Haut ${ }^{*}$

- Juckreiz ${ }^{*}$

- Taubheitsgefühl ${ }^{*}$

- Spannungsgefühl ${ }^{\star}$

- Schweißneigung verstärkt? *

- Gelenk-/Gliederschmerzen an der Hand

\subsection{Statistische Auswertung}

\subsubsection{Methodik}

Die statistischen Auswertungen wurden mit Hilfe von SPSS für Windows, Version 15.0 (SPSS Inc., U.S.A.) durchgeführt. Kontinuierliche Variablen wurden mit Hilfe des Kolmogorov-Smirnov-Tests auf ihre Verteilungsform geprüft. Zum Stichprobenverleich wurden durchgehend nichtparametrische Tests für nicht normalverteilte Stichproben herangezogen.

Beim Vergleich von zwei unabhängigen, nicht normalverteilten Stichproben wurde der Mann-Whitney-U-Test und bei mehr als zwei unabhängigen, nicht normalverteilten Stichproben der $\mathrm{H}$-Test nach Kruskal und Wallis angewendet.

Beim Vergleich von zwei verbundenen Stichproben wurde der WilcoxonTest verwendet. 
Der Korrelation zwischen zwei Parametern wurde durch den Korrelationskoeffizienten nach Spearman's Rho berechnet.

Bei allen durchgeführten Tests erfolgte eine zweiseitige Signifikanzüberprüfung, wobei für alle statistischen Tests ein $p$-Wert $<0,05$ als statistisch signifikant angenommen wurde.

In den ebenfalls mit SPSS erstellten grafischen Darstellungen wurden zur Veranschaulichung der Mittelwerte bei normalverteilten Stichproben Fehlerbalken verwendet, wobei als Streumaß aufgrund der großen Streuungsbreite die Standardfehler aufgeführt wurden. Zur Veranschaulichung der Mediane und Quartilsabstände bei nicht normalverteilten Stichproben wurden Boxplots verwendet. Während in den Boxen der Median sowie die 25.-75. Perzentile aufgetragen sind, entsprechen die T-Balken dem kleinsten und größten Wert, sofern diese keine Ausreißer bzw. Extremwerte sind. Die Ausreißer sind dabei Werte, die zwischen 1 1/2 und 3 Boxlängen außerhalb der Box liegen und sind in den Grafiken als Kreise dargestellt, während Extremwerte, die mehr als drei Boxlängen außerhalb der Box gemessen wurden, als Kreuzchen aufgetragen sind.

\subsubsection{Der Nürnberger Handscore}

Unter Berücksichtigung der ermittelten Ergebnisse aus dem Nürnberger Fragebogen sollte ein Koeffizient definiert werden, der einen Vergleich mit den Ergebnissen des DASH-Fragebogens erlaubt. Dieser Koeffizient heißt Nürnberger Handscore (NFH-Score).

Genau wie beim DASH-Fragebogen sollte auch beim Nürnberger Fragebogen jeder Einzelfrage ein Punktwert zwischen 1 und 5 zugeordnet werden können. Eine derartige Umformung war für einige der verwendeten Fragen unkompliziert durchführbar. In den übrigen Fällen wurde zunächst darauf geachtet, dass eine Gegenläufigkeit von positiv und negativ gestellten Fragen durch eine einheitliche Umkodierung ausgeschlossen werden konnte. Sodann wurde mit Hilfe von Faktorenanalysen Bezug auf 
die ermittelten Antworten zu diesen Fragen genommen und daraus Regeln für eine Umkodierung abgeleitet.

Im Fragebogen wird unter der Ziffer 11 in drei unterschiedlichen Formen zu „Schmerzen“ befragt. Eine Quantifizierung der Aussage ermöglichen allerdings nur die verbalen und numerischen Ratingskalen, so dass diese beiden unabhängigen Variablen (Schmerz/NAS und Schmerz/verbal) in den NFH-Score einfließen konnten. Da dies für die Visuelle Analogskala nicht unmittelbar möglich war, die nötige Berechnung angesichts der Verfügbarkeit der anderen beiden Parameter aber auch nicht erforderlich war, wurde diese Information in der Berechnung des NFH Scores nicht berücksichtigt. Weil außerdem nur eine der beiden Altersangaben aus dem Fragebogen verwendet wurde, fließen wie beim DASH-Score 20 Einzelwerte in den NFH-Score ein.

Dessen Berechnung beginnt mit der Addition dieser 20 Einzelpunktwerte, wobei Summen zwischen 20 und 100 möglich sind. Sodann wird von diesem Rohwert 20 subtrahiert und diese Differenz mit dem Faktor 1,25 multipliziert. Hierdurch ergibt sich für den bestmöglichen Fall $(20$ mal ein Punkt) ein NFH-Score von 0 . Entsprechend berechnet sich für den denkbar schlechtesten Fall (20 mal fünf Punkte) ein Score von 100 Punkten.

Nürnberger Handscore $=($ Rohwert -20$) \times 1,25$

Zur Untersuchung der Anwendbarkeit des Fragebogens wurde eine Reliabilitätsanalyse durchgeführt, bei der der Trennschärfenkoeffizient sowie der Reliabilitätskoeffizient Cronbach's Alpha berechnet wurde. Für alle Items wurde ein Trennschärfenkoeffizient von mindestens 0,17 und ein Cronbach's Alpha von mindestens 0,8 gefordert.

Die Prüfung der Verteilungsform der ermittelten Scorewerte erfolgte mit dem Kolmogorov-Smirnov-Test in der Lilliefors-Variante. Die Korrelationsanalyse erfolgte mit der Bestimmung des Rangkorrelationskoeffizienten nach Spearman's Rho. 


\subsubsection{Plausibilitätsanalyse}

Zur Überprüfung der grundsätzlichen Aussagekraft beider Scores wurden Grundannahmen postuliert und mit statistischen Tests anhand gruppenbildender Kriterien überprüft. Folgende Annahmen wurden formuliert:

- Das Scoringsystem unterliegt keinen geschlechtsspezifischen Einflüssen. (Kriterium 1: Geschlecht)

- Das Scoringsystem unterliegt keinen altersbedingten Einflüssen. (Kriterium 2: Alter)

- Verbrennungsfolgen an der Hand sind letztlich nur in geringem Maße vom zugrundeliegenden Unfallmechanismus abhängig. Das Scoringsystem wird davon nicht beeinflußt. (Kriterium 3: Unfallursache)

- Verbrennungsfolgen an der Hand sind nicht davon abhängig, ob ein Arbeitsunfall vorlag oder ein privater Unfall. Das Scoringsystem wird davon nicht beeinflußt. (Kriterium 4: Arbeitsunfall/privater Unfall)

- Schwerwiegendere Verletzungen führen zu ungünstigeren Folgezuständen als weniger schwerwiegende. Das Scoringsystem spiegelt dies wieder. (Kriterium 5: Verletzungsschwere)

Die Prüfung dieser Annahmen erfolgte durch statistische Tests, bei denen Untergruppen bezogen auf die ermittelten Scorewerte statistisch verglichen wurden. Die Bildung der Untergruppen war für die Kriterien 1 (Geschlecht) und 4 (Arbeitsunfall/privater Unfall) unkompliziert möglich und folgte für das Kriterium 2 (Alter) der Häufigkeitsverteilung. Für das Kriterium 3 (Unfallursache) wurde zunächst eine Hauptkomponentenanalyse durchgeführt. Die Anzahl der Faktoren wurde zur besseren Übersichtlichkeit auf 3 Faktoren begrenzt. Faktorenladungen kleiner als 0,4 wurden aus der Analyse ausgeschlossen.

Das Kriterium 5 (Verletzungsschwere) wurde in differenzierter Form untersucht. Hierzu wurden weitere Grundannahmen postuliert: 
- Die Dauer der Behandlung auf einer Intensivstation ist bei schwerwiegenden Verletzungen höher als bei weniger schwerwiegenden Verletzungen. Eine höhere Zahl an Intensivbehandlungstagen führt zu einem höheren Scorewert. (Kriterium 5a: Dauer der Intensivbehandlung)

- Die Dauer der stationären Behandlung (ohne Intensivstation) ist bei schwerwiegenden Verletzungen höher als bei weniger schwerwiegenden Verletzungen. Eine höhere Zahl an stationären Behandlungstagen führt zu einem höheren Scorewert. (Kriterium 5b: Dauer der stationären Behandlung)

- Eine größere Flächenausdehnung der Gesamtverbrennung führt zu einem höheren Scorewert. (Kriterium 5c: Fläche der Gesamtverbrennung)

- Eine größere Verbrennungstiefe an der Hand führt zu einem höheren Scorewert. (Kriterium 5d: Tiefe der Handverbrennung)

- Sind viele Operationen an der Hand erforderlich, so führt dies zu einem höheren Scorewert. (Kriterium 5e: Anzahl erforderlicher Operationen)

Auch für diese Kriterien wurden in der zuvor beschriebenen Weise Hauptkomponentenanalysen durchgeführt. 


\section{Ergebnisse}

Alle 81 Patienten, die die Einschlußkriterien der Studie erfüllten, haben die zugesandten Fragebögen bearbeitet und zurückgeschickt. Die Rücklaufquote betrug also $100 \%$.

Sämtliche Fragebögen wurden auf Gültigkeit überprüft. Kein Fragebogen musste daraufhin ausgeschlossen werden. Die einzige Auffälligkeit war, dass eine 89-jährige Patientin die Frage 21 des DASH-Fragebogens („Sexuelle Aktivität“) nicht beantwortet hatte.

62 Patienten waren Männer, 19 Frauen. Zum Zeitpunkt des Unfalls waren die Patienten durchschnittlich 38,9 Jahre alt (Minimum 13, Maximum 87 Jahre). 20 Patienten hatten lediglich eine Verletzung ihrer rechten Hand, 12 nur der linken Hand, die übrigen 49 Patienten $(60,5 \%)$ hatten Verbrennungen beider Hände erlitten. Die Flächenausdehnung der verbrannten Körperoberfläche betrug insgesamt durchschnittlich 15,8\% (Minimum 1\%, Maximum 55\%).

Die Patienten wurden durchschnittlich 34,4 Tage in unserer Klinik stationär behandelt, davon 19,9 Tage auf der Intensivstation.

Die Bearbeitung erfolgte durchschnittlich 1,8 Jahre nach dem Unfallzeitpunkt.

\subsection{Analyse der Behandlungsfälle}

\subsubsection{Deskriptive Statistik}

\subsubsection{Ursache der Verbrennungsverletzung}

Die folgende Abbildung illustriert die Angaben zu den jeweiligen Ursachen der Verbrennungsverletzung. In 28 Fällen war dies ein Feuer mit offenen Flammen $(34,6 \%)$, bei 23 Patienten war eine Verpuffung eingetreten $(28,4 \%)$. In 12 Fällen hatte Kleidung gebrannt (14,8\%), 10 Patienten waren Opfer einer Explosion geworden (12,3\%). 27 Patienten hatten einen Arbeitsunfall erlitten (33,3\%). 


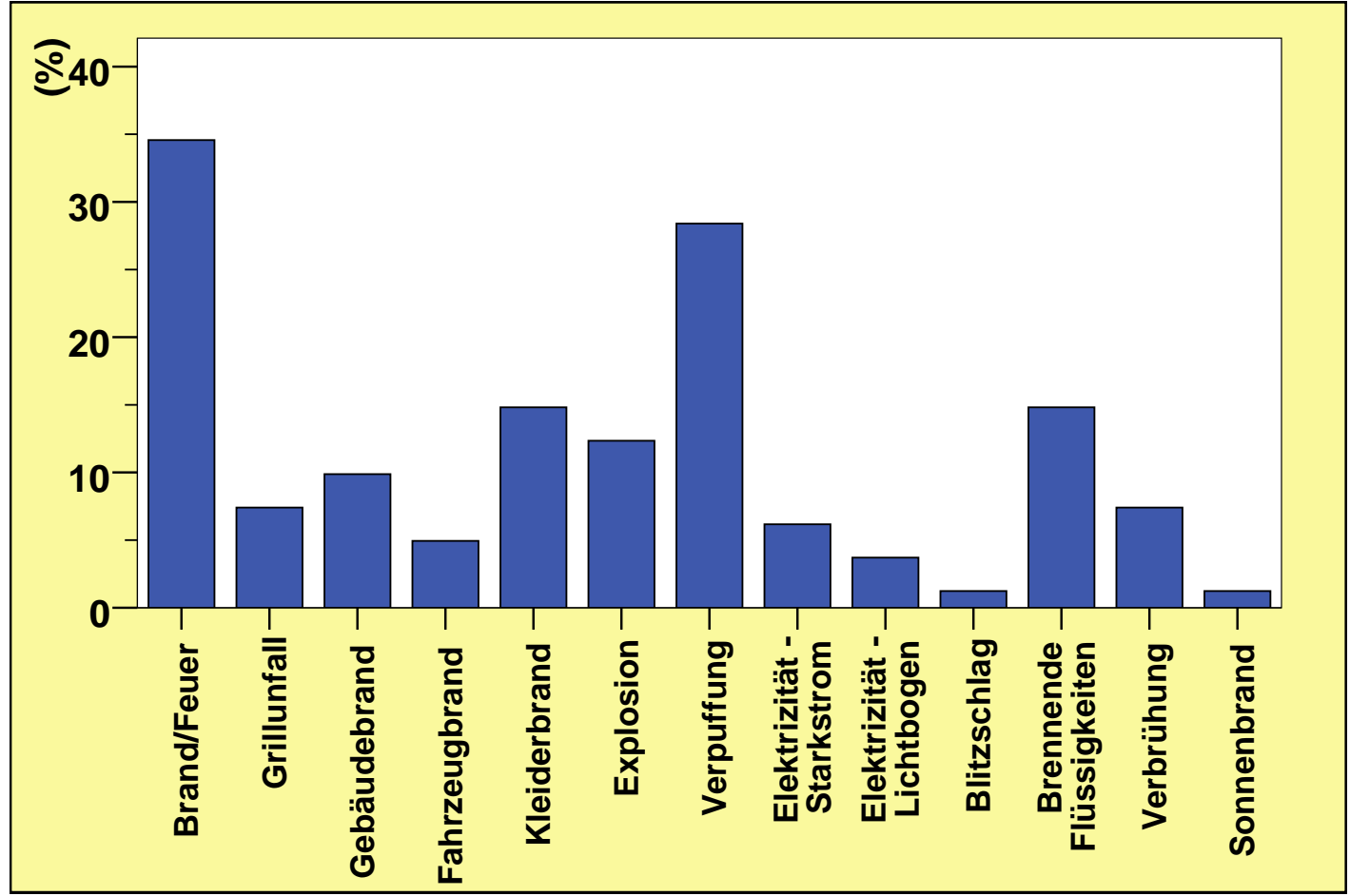

Abb. 19: Ursachen der Verbrennungsverletzung (Mehrfachnennungen sind möglich)

\subsubsection{Tiefenausdehnung der Handverbrennung}

38 Patienten hatten oberflächlich zweitgradige Brandverletzungen an den Händen erlitten $(46,9 \%)$, bei 29 Patienten bestanden tief zweitgradige Handverbrennungen (35,8\%). Drittgradige Verbrennungen fanden sich nur in 11 Fällen 13,6\%). Drei Patienten hatten Verletzungen an beiden Händen, aber in unterschiedlich starker Ausprägung (jeweils oberflächlich bzw. tief zweitgradig).

Nur die 43 Patienten mit tief dermalen Brandverletzungen mussten an den Händen operiert werden. 34 Patienten benötigten lediglich einen Eingriff, sieben Patienten wurden zweimal operiert. Zwei Patienten waren Sonderfälle mit vier bzw. fünf erforderlichen Operationen. 


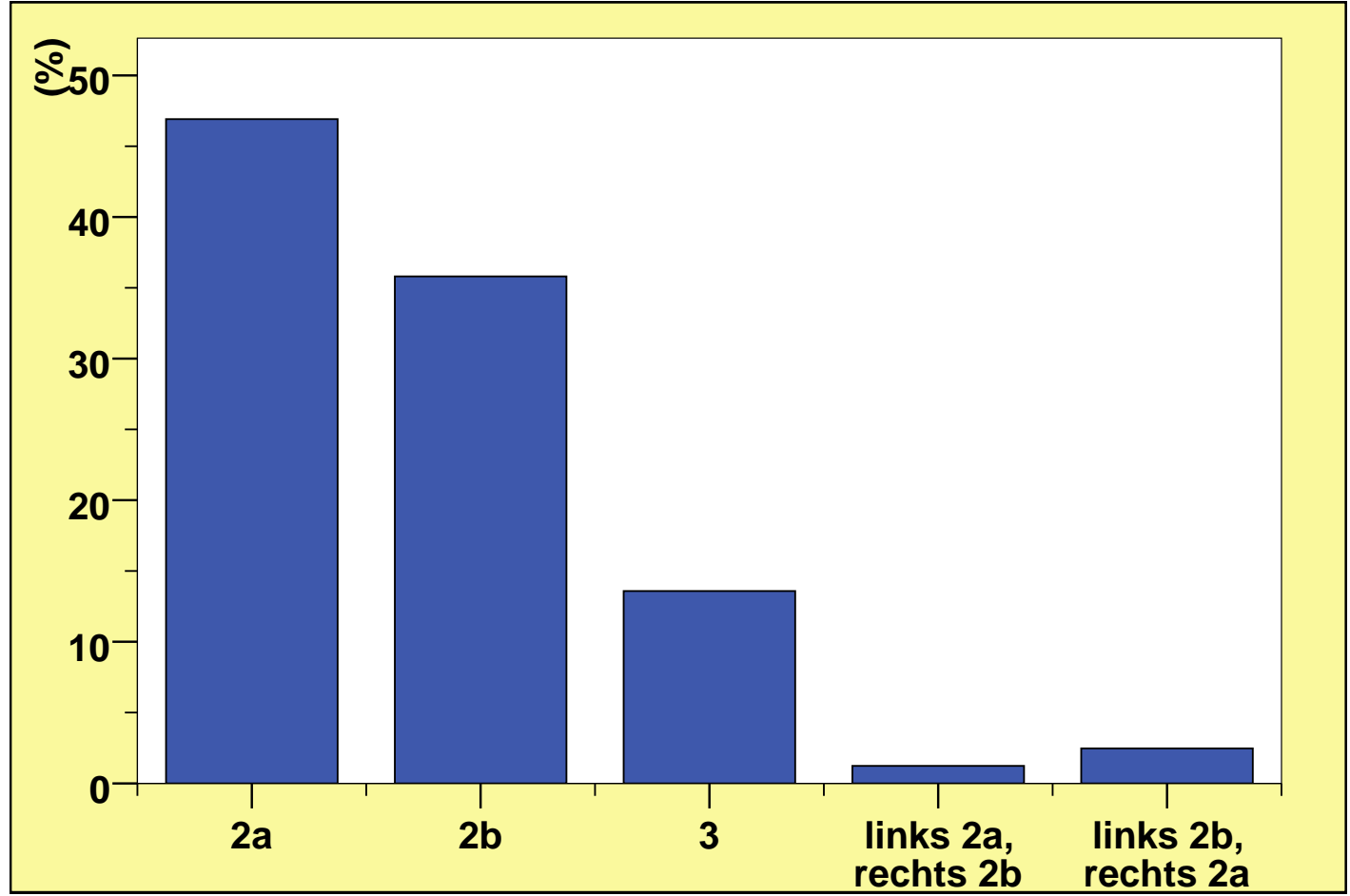

Abb. 20: Tiefenausdehnung der Handverbrennungen

\subsection{DASH-Fragebogen}

\subsubsection{Deskriptive Statistik}

Die Tabellen 1 und 2 geben die Ergebnisse des DASH-Fragebogens wieder. Hierbei wird ersichtlich, dass mehr als $90 \%$ der Patienten angaben, bei den Aktivitäten „Pullover anziehen“ und "Straßenverkehr“ komplett unbeeinträchtigt zu sein. In diesen Belangen bestanden lediglich bei drei Patienten merkliche Beeinträchtigungen.

Der Anteil unbeeinträchtigter Patienten lag für die Items „Schlüssel umdrehen“, „Kochen“, „Betten machen“, „Haare waschen“, „Sex“ und „Schlafstörung“ zwischen 80 und 90\%. Probleme in dieser Hinsicht wurden nur sporadisch angegeben. Vier Patienten (5\%) hatten angegeben, dass aufgrund der Verbrennung keine sexuelle Aktivitäten mehr möglich war.

$70-80 \%$ der Patienten waren beschwerdefrei bezogen auf die Items „Schreiben“, „Gegenstände über das Kopfniveau anheben“, „leichtes Tragen“, „Glühbirnen wechseln“, „Rücken waschen“, „mit dem Messer schneiden“ und „leichte Freizeitaktivitäten“. Die übrigen Patienten gaben 
zwar Schwierigkeiten an, allerdings nur in geringer Ausprägung. Eine Abweichung stellt das Kriterium „Schreiben“ dar, denn 13 Patienten gaben hier zumindest merkliche Einschränkungen an (16,1\%). Mit 12,3\% $(n=10)$ etwas seltener wurden mindestens merkliche Schwierigkeiten beim Messerschneiden und bei leichten Freizeitaktivitäten beklagt, während beim leichten Tragen und Glühbirnenwechseln die mindestens merklichen Schwierigkeiten zu 11,1\% ( $n=9)$ aufgetreten sind.

Tätigkeiten, bei denen mit 60-70\% vergleichsweise seltener eine Beschwerdefreiheit bestand, waren das „Aufstoßen von schweren Türen“, „Putzen“ sowie die „Gartenarbeit“. Auffallend bei diesen schwereren Tätigkeiten ist eine Zunahme an mindestens merklichen Schwierigkeiten, die beim Türaufstoßen 14,8\% $(n=12)$, beim Putzen 19,7\% $(n=16)$ und bei der Gartenarbeit 23,4\% (n=19) der Patienten betraf.

Tätigkeiten, bei denen zwischen 40 und 50\% der Patienten Beschwerden verspürten, waren „Glasöffnen“, „schweres Tragen“, „schwere Freizeitaktivität“, „Freizeitaktivitäten mit überwiegender Armbelastung“ und beim „Spielen von Instrumenten“. Hierbei wurden vermehrt Schmerzen, eine verminderte Spielqualität und eine Abnahme der möglichen Spieldauer gleichermaßen beschrieben. Die hier benannten Tätigkeiten führten in bis zu einem Drittel der Fälle zu mindestens merklichen Schwierigkeiten, wobei diese beim schweren Tragen 29,6\% ( $n=24)$ und bei der Qualität des Instrumentspielens $32,1 \%(n=26)$ betrug. 


\begin{tabular}{|c|c|c|c|c|c|}
\hline & 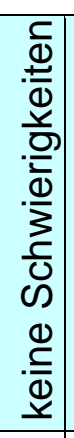 & 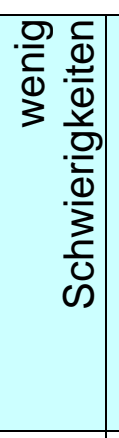 & 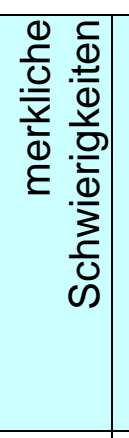 & 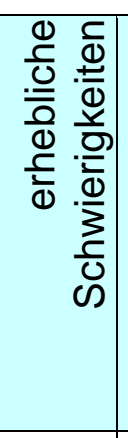 & 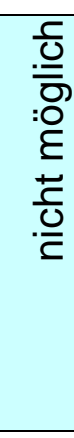 \\
\hline Glas öffnen & 46 & 18 & 11 & 3 & 3 \\
\hline Schreiben & 60 & 8 & 8 & 3 & 2 \\
\hline Schlüssel umdrehen & 67 & 11 & 1 & 2 & \\
\hline Kochen & 68 & 7 & 3 & 1 & 2 \\
\hline Schwere Tür aufstoßen & 52 & 17 & 9 & 2 & 1 \\
\hline Gegenstand über Kopf stellen & 62 & 13 & 3 & 1 & 2 \\
\hline Putzen & 50 & 15 & 10 & 2 & 4 \\
\hline Gartenarbeit & 51 & 11 & 13 & 2 & 4 \\
\hline Betten machen & 67 & 7 & 3 & 2 & 2 \\
\hline leicht Tragen & 60 & 12 & 5 & 1 & 3 \\
\hline schwer Tragen & 42 & 15 & 17 & 4 & 3 \\
\hline Glühbirne wechseln & 64 & 8 & 6 & 1 & 2 \\
\hline Haare waschen & 71 & 5 & 2 & 2 & 1 \\
\hline Rücken waschen & 63 & 12 & 3 & 1 & 2 \\
\hline Pullover anziehen & 73 & 5 & 1 & 2 & \\
\hline Messer schneiden & 57 & 14 & 6 & 3 & 1 \\
\hline Freizeit leicht & 62 & 9 & 6 & 3 & 1 \\
\hline Freizeit schwer & 45 & 17 & 11 & 4 & 4 \\
\hline Freizeit Fokus Arm & 43 & 17 & 10 & 6 & 5 \\
\hline Straßenverkehr & 76 & 3 & & 1 & 1 \\
\hline Sex & 65 & 3 & 6 & 2 & 4 \\
\hline Musik/Sport - wie früher & 43 & 15 & 15 & 2 & 6 \\
\hline Schmerzen bei Musik/Sport & 46 & 20 & 9 & 1 & 5 \\
\hline Qualität Musik/Sport & 44 & 11 & 15 & 5 & 6 \\
\hline Dauer Musik/Sport & 45 & 12 & 13 & 4 & 7 \\
\hline
\end{tabular}

Tab. 1: Ergebnisse der DASH - Items 1 - 21sowie 31 bis 34 


\begin{tabular}{|c|c|c|c|c|c|}
\hline & 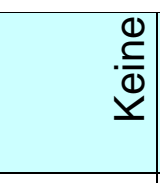 & 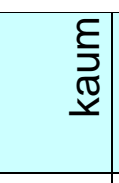 & 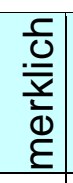 & $\begin{array}{l}\frac{\overline{0}}{0} \\
\frac{.0}{\bar{T}} \\
\frac{0}{0}\end{array}$ & 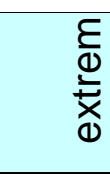 \\
\hline Einschränkung sozialer Kontakte & 52 & 14 & 6 & 6 & 4 \\
\hline Einschränkung Alltag & 45 & 16 & 13 & 4 & 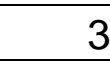 \\
\hline Ruheschmerz & 54 & 19 & 5 & 2 & \\
\hline Schmerz bei aktiven Bewegungen & 39 & 24 & 13 & 3 & \\
\hline Parästhesien & 47 & 18 & 11 & 5 & \\
\hline Schwäche & 40 & 18 & 18 & 5 & \\
\hline Steifheit & 57 & 12 & 10 & 2 & \\
\hline Schlafstörung wg. Schmerz & 69 & 6 & 5 & 1 & \\
\hline & 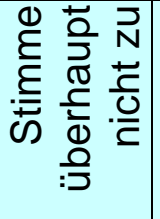 & 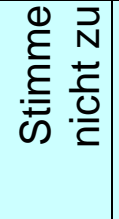 & $\begin{array}{l}\frac{t}{.0} \\
\frac{0}{C} \\
\frac{0}{\overline{0}} \\
3\end{array}$ & 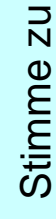 & 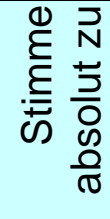 \\
\hline Selbstvertrauen gemindert & 50 & 6 & 8 & 8 & s \\
\hline
\end{tabular}

Tab. 2: Ergebnisse der DASH - Items 22 - 30

Das Ergebnis des Kolmogorov-Smirnov-Tests in der Lilliefors-Variante ergab $p<0,001$. Die ermittelten DASH-Scores waren also nicht normalverteilt.

\subsection{Nürnberger Fragebogen}

\subsubsection{Deskriptive Statistik}

Die Auswertung des Fragebogens ergab folgende Ergebnisse:

- Frage 1: Aktuelles Alter

Mittelwert 40,7 Jahre (Minimum 15, Maximum 89 Jahre)

- Frage 2: Alter zum Unfallzeitpunkt

Mittelwert 38,9 Jahre (Minimum 13, Maximum 87 Jahre)

- Frage 3: Wird der damalige Beruf noch ausgeübt?

Nein: 27; Ja: 51; nicht berufstätig/Rentner: 1

- Frage 4: Einschränkung im Beruf

Keine: 40; Leichte: 25; Starke: 10; Berufsunfähig: 6 
- Frage 5: Ergebnis der Operation/Behandlung

Sehr gut: 58; Gut: 15; Befriedigend: 7; Ausreichend: 1

- Frage 6: Aussehen der Hände

Sehr gut: 34; Gut: 19; Befriedigend: 17;

Ausreichend: 8; Ungenügend: 3

- Frage 7: Hände in der Öffentlichkeit verstecken

Nein: 57; Ja: 24

- Frage 8: Gestikulieren

Unverändert: 37; Mehr als früher: 14; weniger als früher: 30

- Frage 9: Farbunterschiede

Heller: 16; Dunkler: 46; Gleichfarbig: 10; Teils/teils: 9

- Frage 10: Sensibilität der Hände

Durchschnittlich $81,7 \%$

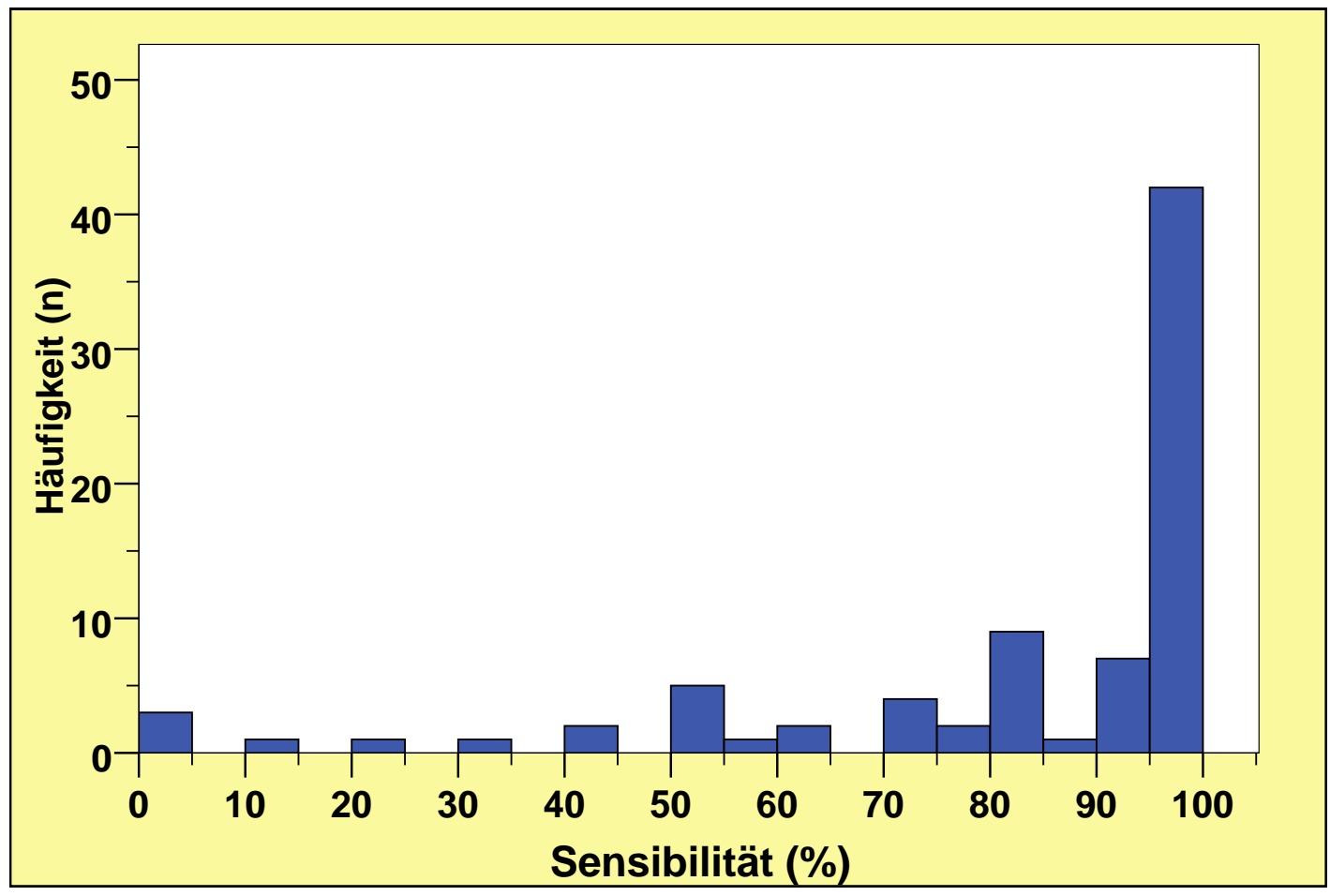

Abb. 21: Häufigkeitsverteilung der Angaben zur Sensibilität

- Frage 11: Schmerz/verbal

Kein: 40; Leicht: 24; Mäßig: 13; Stark: 4 
- Frage 11: Schmerz/NAS

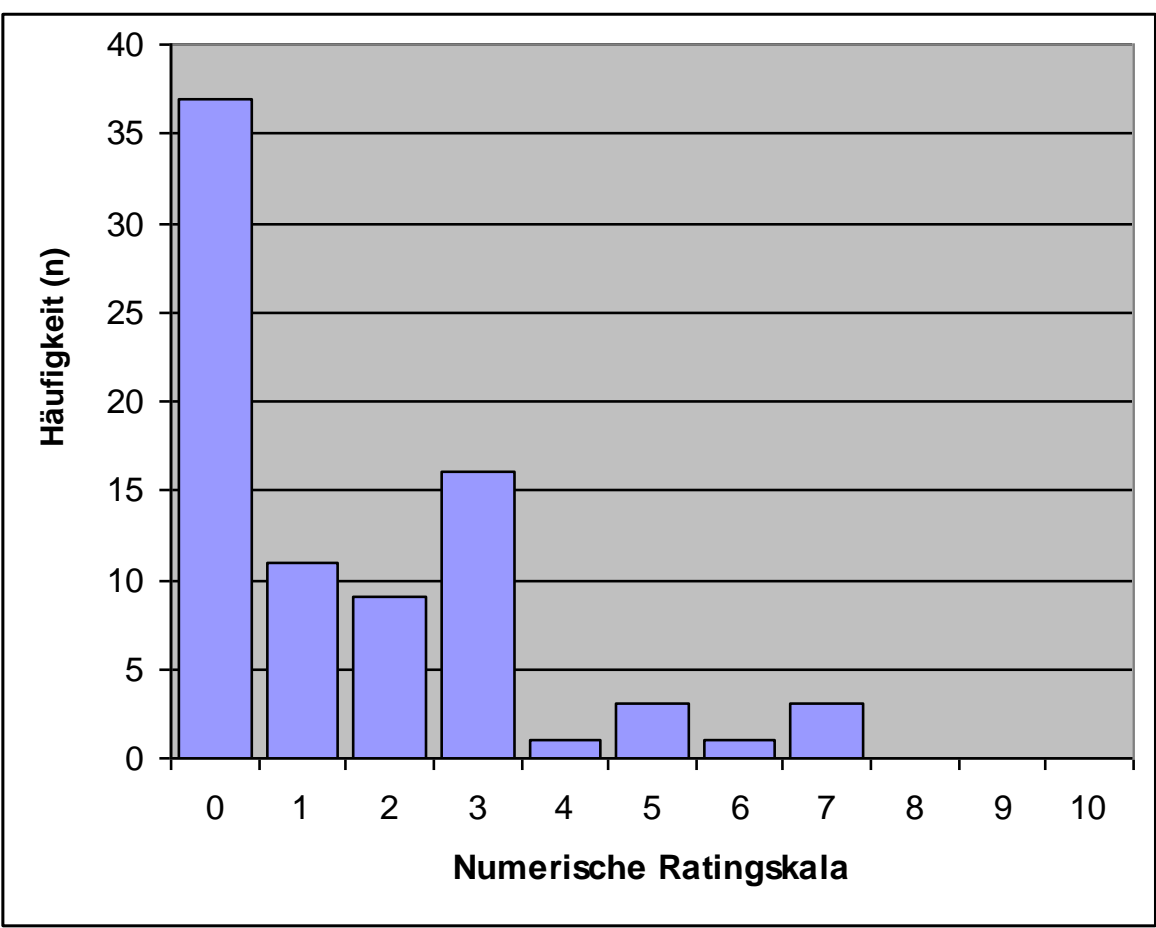

Abb. 22: Häufigkeitsverteilung der Angaben zu Schmerzen nach der Numerischen Skala

- $\quad$ Frage 12: Kälteempfindlichkeit

Erhöht: 55; Unverändert: 23; Vermindert: 3

- Frage 13: Wärmeempfindlichkeit

Erhöht: 30; Unverändert: 38; Vermindert: 13

- Frage 14: Trockenheit

Erhöht: 48; Unverändert: 27; Vermindert: 6

- Frage 15: Verletzlichkeit

Erhöht: 46; Unverändert: 33; Vermindert: 2

- $\quad$ Frage 16: Juckreiz

Nein: 51; Ja: 30

- $\quad$ Frage 17: Taubheitsgefühl

Nein: 59; Ja: 22 
- Frage 18: Spannungsgefühl

Nein: 44; Ja: 37

- Frage 19: Schweißneigung

Erhöht: 1; Unverändert: 65; Vermindert: 15

- Frage 20: Gelenkschmerzen

Nein: 63; Ja: 18

4.3.2. Definition des Nürnberger Handscores

Unter Berücksichtigung der ermittelten Resultate führte die Faktorenanalyse zu folgenden Umformungsregeln:

- Fragen 1 und 2: Alter

Für den NFH-Score wurde nur das Alter zum Unfallzeitpunkt (nicht das ebenfalls erfragte Alter zum Zeitpunkt der Befragung) berücksichtigt. Die bei unseren 81 Patienten ermittelten Altersangaben ergaben Quartilen von 27,5, 41 und 50,5 Jahre. Die Grundannahme zu diesem Kriterium war: je älter der Patient, desto ungünstiger das Ergebnis und desto höher die entsprechende Punktzahl. Die Punktwertzuordnung erfolgte daher sinnvollerweise folgendermaßen:

- Alter bis 27,5 Jahre:

1 Punkt

- Alter über 27,5 Jahre und unter 50,5 Jahre:

3 Punkte

- Alter über 50,5 Jahre:

5 Punkte

- Frage 5: Ergebnis der Operation

Grundsätzlich waren im Fragebogen Antwortmöglichkeiten von 1 bis 6 möglich (Schulnotensystem). Die tatsächlich angegebenen Antworten waren aber überwiegend sehr gute und gute Einschätzungen. Aufgrund der Quartilen (1, 1 und 2) erfolgte die folgende Festlegung:

- Antwort „Sehr gut":

1 Punkt

- Antwort „Gut":

3 Punkte

- Antwort „Befriedigend“ und schlechter:

5 Punkte 
- Frage 6: Aussehen der Hände

Auch bei dieser Frage standen sechs Antwortmöglichkeiten zur Auswahl. Schlechte Einschätzungen (5 oder 6 ) waren aber fast überhaupt nicht getroffen worden (nur drei Mal eine 6). Die Quartilen lauteten 1, 2 und 3, so dass auch hier wieder die folgende Umformung festgelegt wurde:

- Antwort "Sehr gut":

1 Punkt

- Antwort „Gut":

3 Punkte

- Antwort „Befriedigend“ und schlechter:

5 Punkte

\section{- Frage 10: Sensibilität der Hände}

Hier konnten Prozentwerte frei angegeben werden, wobei 100\% dem vorherigen Normalzustand entsprachen. Eine hohe Prozentzahl wurde also zu einer geringen Punktzahl und umgekehrt. Bei unseren 81 Patienten ergaben sich folgende Quartile: 72,5, 95 und 100. Daraus ergab sich die folgende Umformung:

- $100 \%$ :

1 Punkt

- 73 bis $99 \%$ :

3 Punkte

- unter $73 \%$ :

5 Punkte

\section{- Frage 11: Schmerz/NAS}

Die Auswertung beschränkt sich hier auf die Betrachtung der Angaben zur numerischen Ratingskala. Im Fragebogen konnten Werte zwischen 0 und 10 angegeben werden, wobei 0 einer Schmerzfreiheit entsprach. Bei unseren 81 Patienten fanden sich folgende Quartile: 0, 1 und 3. Daraus ergab sich die folgende Umformung:

- Antwort „0“:

1 Punkt

- Antwort „1“:

3 Punkte

- Antwort „2“ und schlechter:

5 Punkte

Die Antworten zu den übrigen Fragen ließen sich wie folgt unkompliziert umkodieren, wobei die kleinste Punktzahl stets dem bestmöglichen Ergebnis entsprechen musste: 
- $\quad$ Frage 3: Berufsausübung

- ja:

1 Punkt

- nein:

3 Punkte

- kein Beruf/arbeitslos/Rentner:

5 Punkte

- Frage 4: Einschränkungen im Beruf

- nein:

1 Punkt

- leicht:

2 Punkte

- stark:

3 Punkte

- berufsunfähig:

4 Punkte

- Frage 7: Hände in der Öffentlichkeit verstecken

- nein:

1 Punkt

- ja:

5 Punkte

- $\quad$ Frage 8: Gestikulieren

- mehr:

1 Punkt

- weniger:

5 Punkte

- Frage 9: Farbunterschiede

- Keine Unterschiede:

1 Punkt

- $\quad$ Alle anderen (,heller“ oder „dunkler“):

5 Punkte

- Frage 11: Schmerz/verbal

- keine:

1 Punkt

- leichte:

2 Punkte

- mäßige:

3 Punkte

- starke:

4 Punkte

- maximal vorstellbare:

5 Punkte

- $\quad$ Frage 12: Kälteempfindlichkeit

- unverändert:

1 Punkt

- $\quad$ Alle anderen („erhöht" oder „vermindert“):

5 Punkte

- Frage 13: Wärmeempfindlichkeit

- unverändert:

1 Punkt

- $\quad$ Alle anderen („erhöht" oder „vermindert“):

5 Punkte 
- $\quad$ Frage 14: Trockenheit

- Keine Unterschiede:

1 Punkt

- $\quad$ Alle anderen („erhöht" oder „vermindert“):

5 Punkte

- $\quad$ Frage 15: Verletzlichkeit

- Keine Unterschiede:

1 Punkt

- $\quad$ Alle anderen („erhöht" oder „vermindert"):

5 Punkte

- Fragen 16: Juckreiz/ 17: Taubheits-/ 18 Spannungsgefühl

- Nein:

1 Punkt

- Ja:

5 Punkte

- $\quad$ Frage 19: Schweißneigung

- Unverändert:

1 Punkt

- $\quad$ Alle anderen („erhöht" oder „vermindert“):

5 Punkte

- $\quad$ Frage 20: Gelenkschmerzen

- Nein:

1 Punkt

- Ja:

5 Punkte

Eine tabellarische Übersicht $\mathrm{zu}$ diesem Fragenkatalog ist mit dem entsprechenden Bewertungsschema der Anlage 3 zu entnehmen.

Die hier vorgestellte Umformung der Ergebnisse aus dem Nürnberger Fragebogen führte bei Auswertung der Antworten unserer 81 ehemaligen Patienten zu einem durchschnittlichen NFH-Score von 41,2 (Abb. 23). 


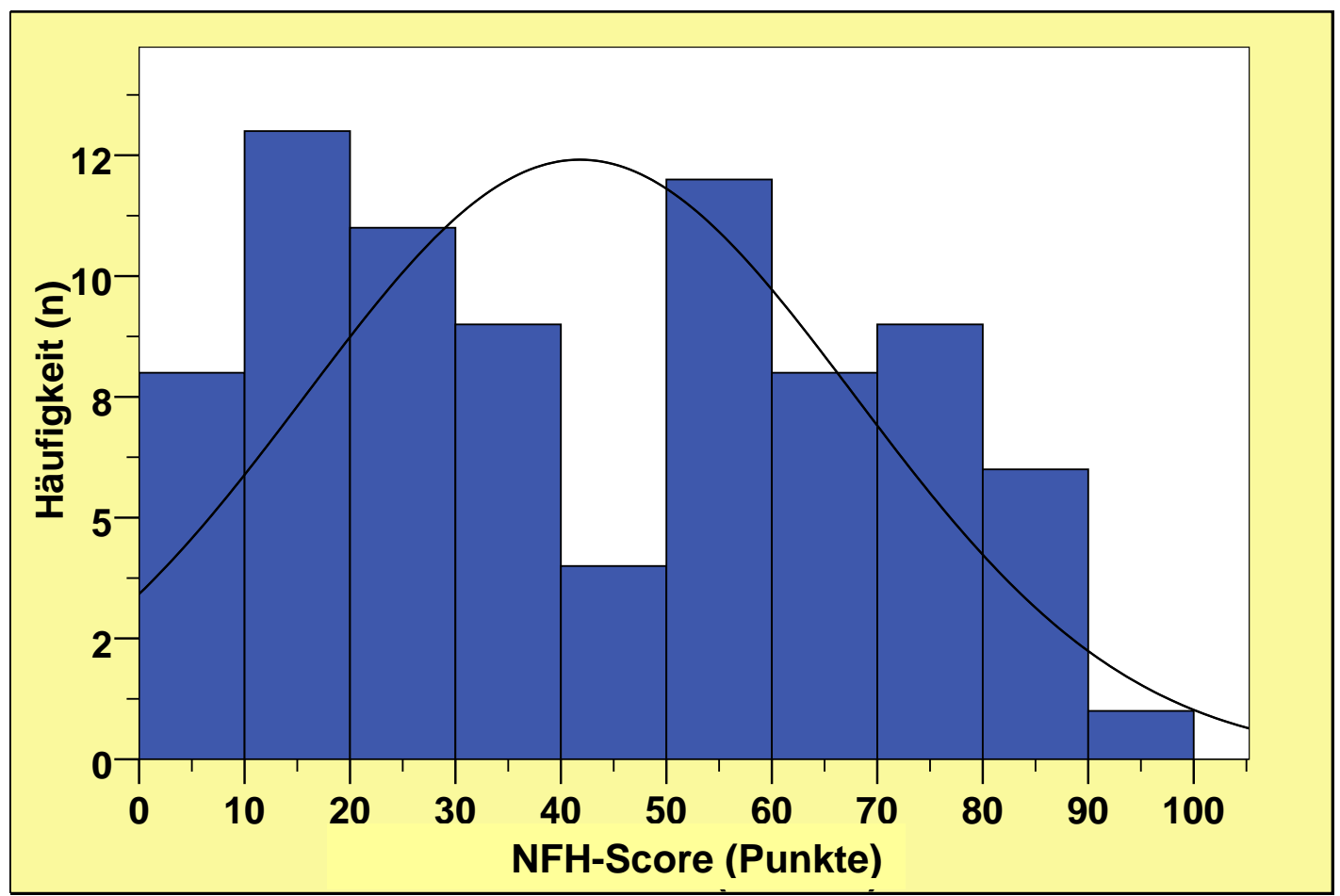

Abb. 23: Häufigkeitsverteilung NFH-Score in der Stichprobe

Zur Prüfung der Verteilungsform erfolgte der Kolmogorov-Smirnov-Test in der Lilliefors-Variante. Mit $p=0,015$ bestand eine signifikante Abweichung von der Normalverteilung.

\subsection{Reliabilitätsanalyse von DASH-Score und NFH-Score}

Bei der Reliabilitätsanalyse ergaben alle Parameter einen Trennschärfenkoeffizient von mindestens 0,17. Cronbach's Alpha lag für alle geprüften Items also deutlich über den geforderten 0,8. Daher musste auch kein Item aus dem Nürnberger Fragebogen ausgeschlossen werden (Tab. 3). 


\begin{tabular}{|c|c|c|c|c|}
\hline & $\begin{array}{c}\text { Skalen- } \\
\text { mittelwert, } \\
\text { wenn Item } \\
\text { weg- } \\
\text { gelassen }\end{array}$ & $\begin{array}{c}\text { Skalen- } \\
\text { varianz, } \\
\text { wenn Item } \\
\text { weg- } \\
\text { gelassen }\end{array}$ & $\begin{array}{l}\text { Trenn- } \\
\text { schärfe- } \\
\text { Index }\end{array}$ & $\begin{array}{l}\text { Cronbachs } \\
\text { Alpha, wenn } \\
\text { Item weg- } \\
\text { gelassen }\end{array}$ \\
\hline \multirow{20}{*}{$\begin{array}{l}\text { Alter aktuell } \\
\text { Beruf } \\
\text { Einschränkung im } \\
\text { Beruf } \\
\text { Ergebnis OP } \\
\text { Ergebnis Optik } \\
\text { Scham } \\
\text { Gestik } \\
\text { Hautfarbe } \\
\text { Sensibilität (\%) } \\
\text { Schmerz verbal } \\
\text { Schmerz (NAS) } \\
\text { Kälteempfinden } \\
\text { Wärmeempfinden } \\
\text { Trockenheit } \\
\text { Verletzlichkeit der } \\
\text { Haut } \\
\text { Juckreiz } \\
\text { Taubheit } \\
\text { Spannungsgefühl } \\
\text { Schweißneigung } \\
\text { Gelenkschmerz }\end{array}$} & 50,44 & 421,675 & ,178 & 918 \\
\hline & 51,63 & 414,386 & 399, & ,914 \\
\hline & 51,59 & 403,444 & 645 & ,910 \\
\hline & 51,68 & 403,521 & ,540 & ,912 \\
\hline & 50,59 & 382,019 & ,713 & ,907 \\
\hline & 51,26 & 390,169 & ,557 & 911 \\
\hline & 50,27 & 409,400 & ,254 & ,920 \\
\hline & 48,94 & 416,534 & ,291 & ,916 \\
\hline & 50,91 & 378,930 & 815 & ,905 \\
\hline & 51,68 & 406,496 & ,738 & ,910 \\
\hline & 50,54 & 375,326 & ,763 & ,906 \\
\hline & 49,58 & 385,597 & ,633 & 909 \\
\hline & 50,32 & 382,671 & 602 & 910 \\
\hline & 49,78 & 382,725 & ,643 & ,909 \\
\hline & 50,07 & 381,869 & ,624 & ,909 \\
\hline & 50,96 & 383,436 & 615 & 910 \\
\hline & 51,36 & 384,608 & ,658 & ,909 \\
\hline & 50,62 & 370,489 & ,771 & ,905 \\
\hline & 51,65 & 401,004 & 475 & ,913 \\
\hline & 51,56 & 394,925 & ,546 & ,911 \\
\hline
\end{tabular}

Tab. 3: Item-Skala-Statistiken beim Nürnberger Handscore

Für alle 20 Items war Cronbach's Alpha 0,915.

\subsection{Plausibilitätsanalyse}

Der Altersmedian lag in unserem Kollektiv bei 41 Jahren. Die Aufteilung in Untergruppen mit Bezug auf das Alter (Kriterium 2) erfolgte dementsprechend wie folgt: $</=41$ Jahre und $>41$ Jahre.

Aufgrund der Faktorenanalyse ergaben sich für das Kriterium 3 (Unfallursache) die drei Untergruppen „Brand“, „Verpuffung“, und „sonstige“.

Der Median der Dauer der Intensivbehandlung betrug in unserem Kollektiv 11 Tage. Die Aufteilung in Untergruppen mit Bezug auf die Dauer der 
Intensivbehandlung (Kriterium 5a) erfolgte dementsprechend wie folgt: "</=11 Tage" und "> 11 Tage“.

Der Median der Dauer der stationären Behandlung betrug in unserem Kollektiv 16 Tage. Die Aufteilung in Untergruppen mit Bezug auf die Dauer der Intensivbehandlung (Kriterium $5 b$ ) erfolgte dementsprechend wie folgt: "</=16 Tage“ und „> 16 Tage“.

Der Median der Flächenausdehnung der Gesamtverbrennung betrug in unserem Kollektiv 13,5\% vKOF. Die Aufteilung in Untergruppen mit Bezug auf dieses Merkmal (Kriterium 5c) erfolgte dementsprechend wie folgt: "</=13,5\% vKOF“ und „> 13,5\% vKOF“

Die Aufteilung in Untergruppen mit Bezug auf die Tiefe der Handverbrennung (Kriterium 5d) erfolgte anhand des klinischen Befundes: „2a“, „2b“ und „3“

Die Aufteilung in Untergruppen mit Bezug auf die Anzahl der erforderlichen Operationen (Kriterium 5e) erfolgte aufgrund der Faktorenanalyse wie folgt: „maximal eine Operation“ und „zwei oder mehr Operationen“.

$\mathrm{Da}$ es sich jeweils um unabhängige Stichproben handelte und die Gesamtergebnisse sowohl der DASH-Scores wie auch der NFH-Scores nicht normalverteilt waren, wurde beim Vergleich zwischen zwei Untergruppen der Mann-Whitney-U-Test und beim Vergleich von drei Untergruppen der Kruskal-Wallis-H-Test angewendet.

Tab. 4 zeigt in der Zusammenfassung die Ergebnisse dieser Testverfahren. 


\begin{tabular}{|l|c|r|r|}
\hline & & DASH Score & NFH Score \\
\hline Geschlecht & Männer vs. Frauen & $\mathrm{p}>0,05$ & $\mathrm{p}>0,05$ \\
Alter & $</=41$ a vs. $>41 \mathrm{a}$ & $\mathrm{p}>0,05$ & $\mathrm{p}>0,05$ \\
Ursache & Brand, Verpuffung, & $\mathrm{p}>0,05$ & $\mathrm{p}>0,05$ \\
& sonstige & $\mathrm{p}>0,05$ & $\mathrm{p}>0,05$ \\
Arbeitsunfall & AU vs. Freizeitunfall & $\mathbf{p}<0,001$ & $\mathbf{p}<\mathbf{0 , 0 0 1}$ \\
Dauer intensiv & $</=11$ d vs. $>11 \mathrm{~d}$ & $\mathbf{p}<0,001$ & $\mathbf{p}<\mathbf{0 , 0 0 1}$ \\
Dauer & $</=16$ d vs. $>16 \mathrm{~d}$ & $\mathbf{p}<\mathbf{0 , 0 5}$ & $\mathbf{p}<\mathbf{0 , 0 5}$ \\
stationär & $</=13,5 \%$ vs. $>13,5 \%$ & $\mathbf{p}<0,001$ & $\mathbf{p}<\mathbf{0 , 0 0 1}$ \\
vKOF & 2a, 2b, 3 & $\mathbf{p}<0,001$ & $\mathbf{p}<\mathbf{0 , 0 0 1}$ \\
Tiefe & $</=1$ OP vs. $>1$ OP & &
\end{tabular}

Tab. 4: Plausibilitätstests der beiden Scores

\subsection{Vergleich zwischen DASH-Score und NFH-Score}

\subsubsection{Korrelationsanalyse}

Da sich die NFH-Scorewerte definitionsgemäß zwischen 0 und 100 bewegen müssen und dies beim DASH-Score genauso ist, konnte ein direkter Vergleich durchgeführt werden. Bei der Beurteilung der Korrelation zwischen den beiden nicht normalverteilten Stichproben ergab Spearman's Rho einen Rangkorrelationskoeffizienten von 0,85. Zwischen beiden Stichproben besteht also eine hohe Korrelation, die sich graphisch auch im Verlauf der Regressionsgeraden im Bezug zur Punktwolke des Streudiagramms zeigt. Beide Scores drücken also sehr ähnliche Inhalte aus (Abb. 24).

Auffällig ist allerdings, dass die Fälle mit gleichartigem DASH-Score „0“ stark unterschiedliche NFH-Scores aufweisen. Dieser Score ist auffallend oft schlechter als „0“. 


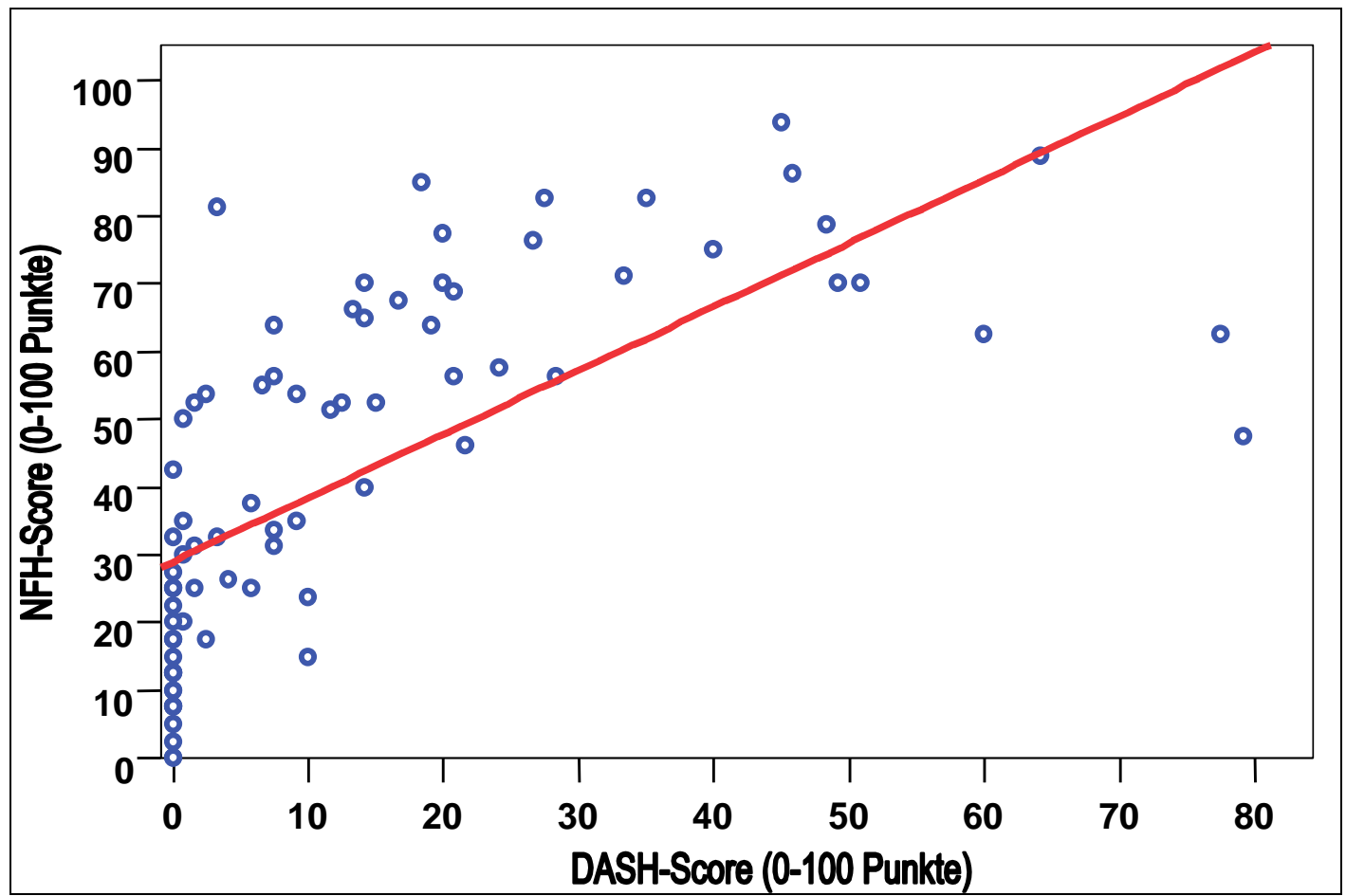

Abb. 24: Streudiagramm zur Darstellung des Zusammenhangs zwischen den Scores

\subsubsection{Statistischer Vergleich der beiden Scores}

Vergleicht man die beiden Scores, so betrachtet man verbundene Stichproben. Da diese im vorliegenden Fall nicht normalverteilt waren, wurde hierfür der Wilcoxon Test angewendet.

Die aus der Boxplot-Grafik bereits anzunehmende Aussage, dass die beiden Scoringsysteme zu unterschiedlichen Ergebnissen führten, bestätigte sich im Wilcoxontest mit höchster Signifikanz: $p<0,001$.

Betrachtet man ausschließlich die Fälle, in denen lediglich eine oberflächlich zweitgradige Verbrennung der Hand bestanden hatte, so wurden die Unterschiede noch deutlicher (Abb. 26). Bei diesen Patienten lag der Mittelwert des DASH-Scores bei 3, während der Mittelwert des NFH-Scores 22,4 betrug. Der Wilcoxontest belegte die Verschiedenheit wiederum mit höchster Signifikanz $(p<0,001)$. 


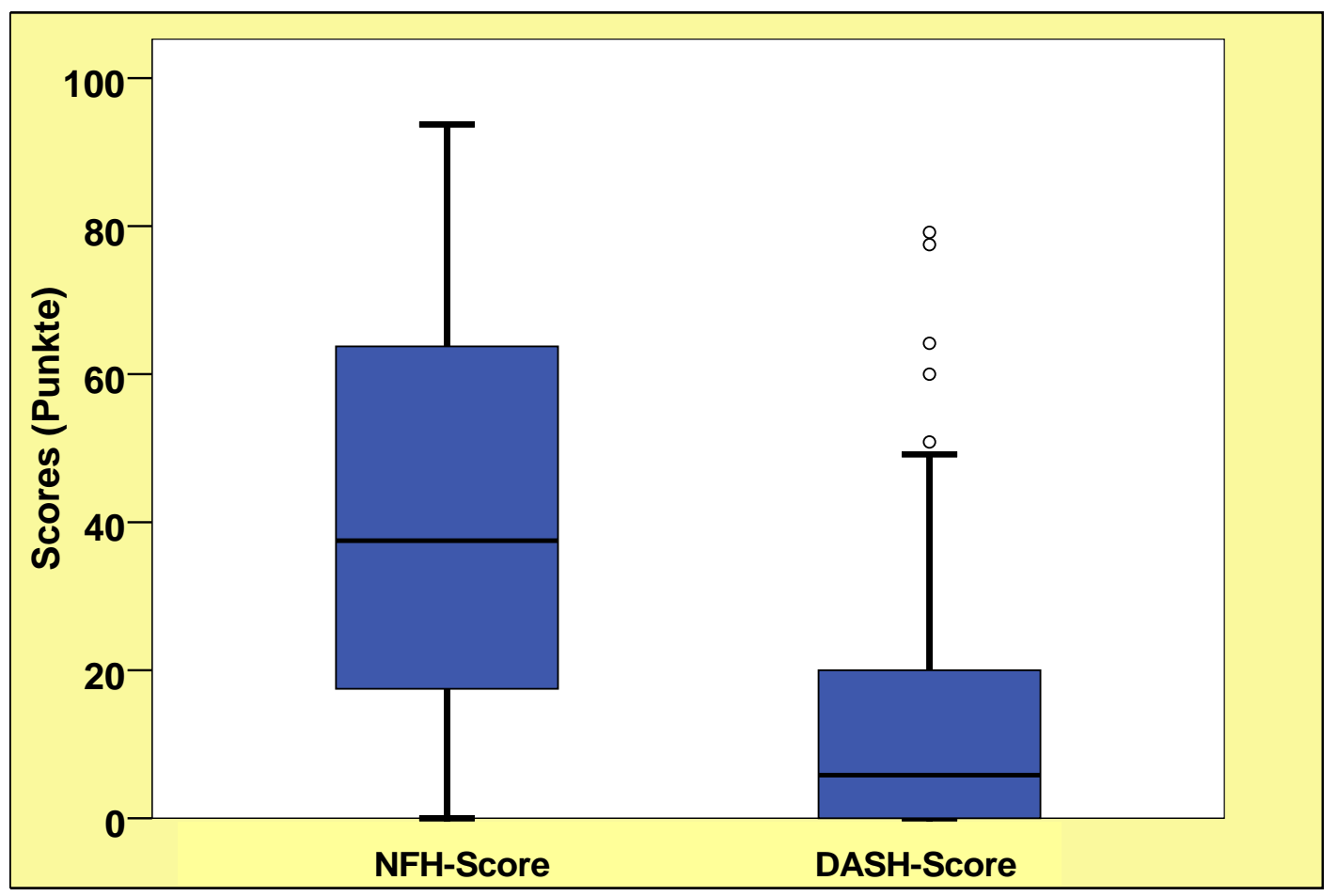

Abb. 25: Boxplots zur Darstellung der Verteilung der beiden Scores

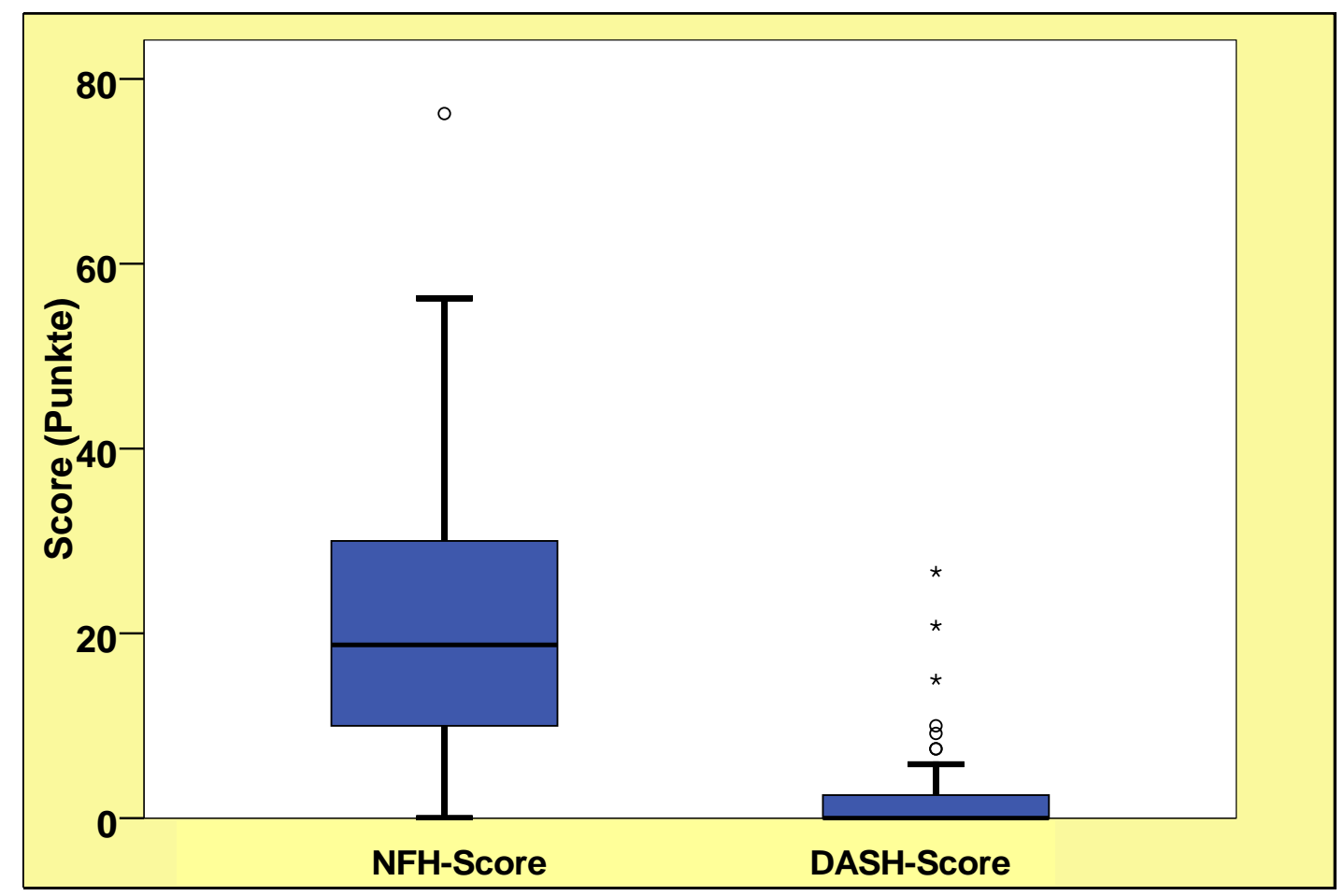

Abb. 26: Boxplots zur Darstellung der Verteilung der beiden Scores, nur 2a 
Die übrigen 43 Fälle, also Scores der Patienten mit den tiefer greifenden Verbrennungsverletzungen an der Hand Grad $2 b$ oder 3 , wurden in beiden Scoringsystemen mit ähnlichem Differenzierungsgrad abgebildet (Abb. 27). Die beiden Scores waren erneut höchstsignifikant verschieden $(p<0,001)$.

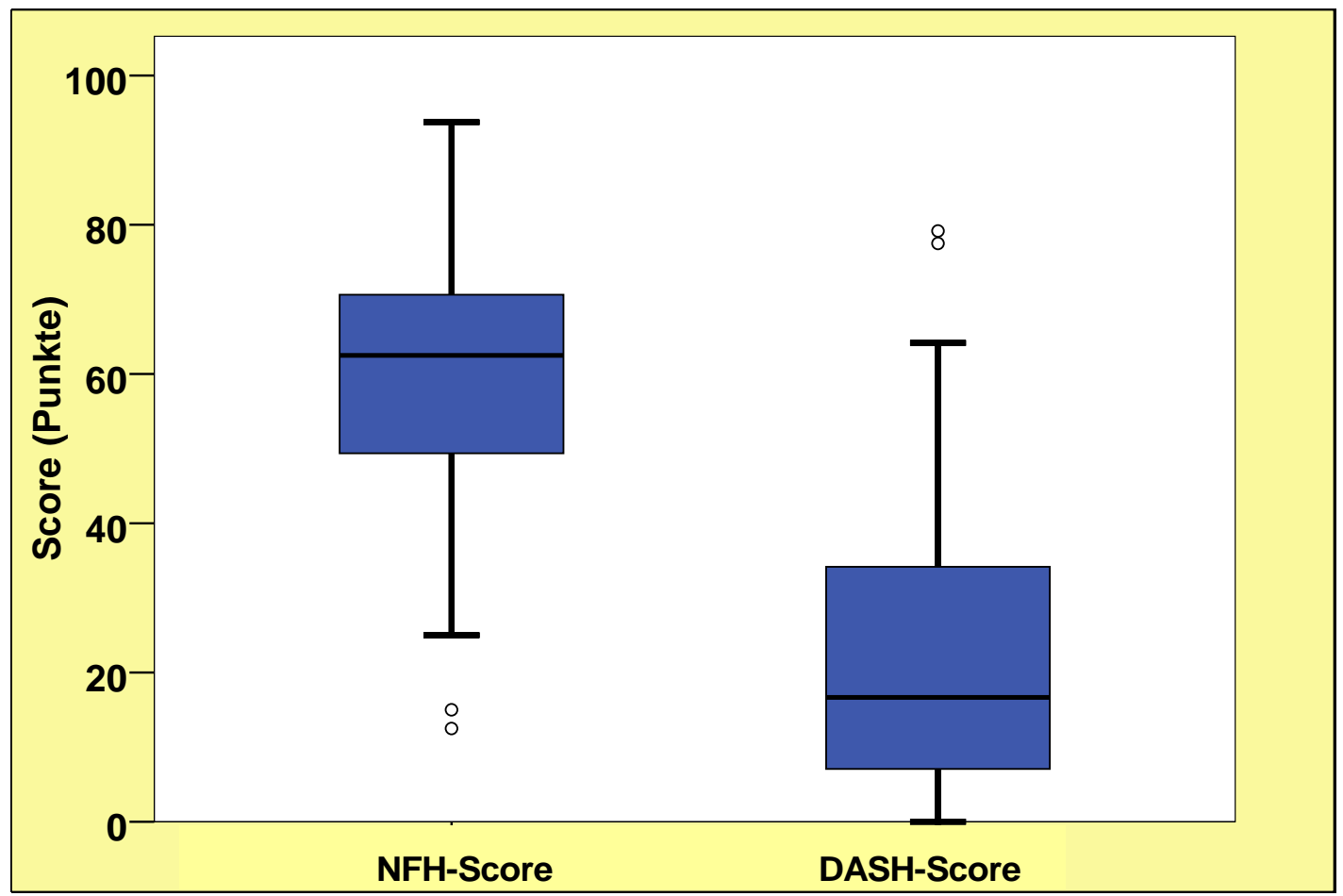

Abb. 27: Boxplots zur Darstellung der Verteilung der beiden Scores, nur 2b und 3 


\section{Diskussion}

Brandverletzungen hinterlassen immer Folgen wie Narben, aber auch Funktionsstörungen. Nachteilig sind derartige Folgen insbesondere im Gesicht, im Genitalbereich, vor allem aber an den Händen. Narben beeinträchtigen die Handfunktion und führen zu Entstellungen [11, 21]. Es ist von besonders großer Bedeutung, die Entwicklung von narbigen Kontrakturen nach Verbrennungen an der Hand zu vermeiden [35], wobei dies auch bei sachgerechter medizinischer Behandlung nicht immer gelingt [2]. Wenn kontrakte Narben bestehen, können unterschiedliche chirurgische Behandlungsverfahren zur Korrektur angewendet werden [41]. Grundsätzlich sollte dies allerdings erst nach Ablauf der Narbenmaturation erfolgen, die üblicherweise ein Jahr in Anspruch nimmt [29]. Bei Funktionseinschränkungen der Fingergelenke sollte dagegen frühzeitig korrigiert werden. Neben der Wundbehandlung ist an der Hand vor allem eine intensive, geradezu aggressive Funktonstherapie von ausschlaggebender Bedeutung [54].

Neben der Qualität der Behandlung bestimmt vor allem die Verbrennungstiefe das dauerhafte Ergebnis einer Brandverletzung an der Hand [34, 59]. Je tiefer der Schaden ist, desto mehr Hautanteile gehen verloren [39]. Auch durch moderne plastisch-chirurgische Techniken lassen sich diese nicht immer adäquat ersetzen [23, 51, 64]. Funktionelle Einschränkungen sind ebenso wie ästhetische und psychosoziale Nachteile nach tiefen Verletzungen häufiger zu erwarten als nach oberflächlichen Läsionen [55]. In dieser Hinsicht spielt die Flächenausdehnung eine geringere Rolle. Besonders an der Hand können bereits kleinflächige Verbrennungen erhebliche Auswirkungen haben, wenn tiefere Hautschichten betroffen sind. Oberflächliche Verletzungen heilen in der Regel günstiger $a b$, insbesondere müssen sie nicht zwingend operiert werden. Sofern es gelingt, eine solche Wunde effektiv vor Sekundärinfektionen zu schützen, sollte sie in wenigen Tagen weitestgehend folgenlos abheilen. Kommt es nach weniger tiefreichenden 
Verletzungen dennoch zu funktionell und ästhetisch relevanten Folgen, so sind zumeist eine inadäquate Behandlung oder aber Infektkomplikationen verantwortlich zu machen [36].

Die in unserem Kollektiv erfaßten 38 als oberflächlich zweitgradig verletzt eingestuften Patienten mussten nicht operiert werden. Die Einschätzung der Verbrennungstiefe ist also ausnahmslos korrekt erfolgt. Allerdings geben zur Frage 6 des Nürnberger Fragebogens, die nach dem Aussehen der Hände fragt, nur 34 Patienten an, sehr zufrieden zu sein. Vier als oberflächlich verletzt eingestufte Patienten haben also zumindest moderat sichtbare Verletzungsfolgen davongetragen. Dies verdeutlicht, dass die initiale Einschätzung der Verbrennungstiefe korrekt sein kann, eine tatsächlich folgenlose Abheilung aber dennoch nicht immer eintritt.

Interessant ist dabei, dass der hohe Grad der Zufriedenheit mit dem erreichten Ergebnis durch Pigmentunterschiede zwischen ehemals verletzter und umgebender Haut offenbar nicht getrübt wird. Immerhin geben nur zehn Patienten an, dass die Verletzungszone gleichfarbig ist (deutlich häufiger ist dieses Areal dunkler $(n=46)$ oder auch heller $(n=16)$ ). 24 Patienten stellen also Pigmentveränderungen fest, sind aber dennoch mit dem Abheilungsergebnis sehr zufrieden.

Scoringsysteme können Verletzungsfolgen klassifizieren und dadurch zu einer einheitlichen Nomenklatur beitragen. Sie bilden Grundlagen für statistische Erfassungen und Auswertungen. Außerdem lassen sich aus manchen Scoring-Systemen Behandlungsstrategien ableiten, Prognosen erstellen oder Begründungen für gutachterliche Einschätzungen von Verletzungsfolgen finden. Derartige Systeme sind daher seit langem etabliert und gebräuchlich, beispielsweise in der Intensivmedizin (APACHE [31], GCS [57]) der Traumatologie (ISS [1], HTS [4, 42]), der Onkologie (WPSS [9]) usw.. In der Verbrennungsmedizin ist der ABSI-Score zur Bestimmung der Sterbewahrscheinlichkeit gebräuchlich [58]. Eine 1978 speziell für Brandverletzungen vorgeschlagene Systematik zur Erfassung 
der Verletzungsschwere bei Brandverletzungen hat sich dagegen nicht durchgesetzt [14].

Scoringsysteme werden ständig weiterentwickelt und verbessert [20, 33, 46], denn sie haben auch Schwächen. Ihre Aussagen lassen sich nicht einheitlich auf eine breite Population übertragen und neben klinischpathologischen Kriterien sollen zukünftig verstärkt auch biologische Aspekte berücksichtigt werden [19].

Seit etwa zehn Jahren ist es in den deutschsprachigen Fachgesellschaften für Handchirurgie üblich, die Ergebnisse ihrer klinischen Untersuchungen immer auch mit Hilfe des DASH-Scores zu evaluieren.

\subsection{DASH-Score}

Der in der Handchirurgie seit langem etablierte Disability of Arm, Shoulder, Hand-Score (DASH-Score) kann auch zur Beurteilung der Folgen von Verbrennungsverletzungen der Hand verwendet werden [6, 18, 23, 40]. Der zugrundeliegende Fragebogen ist von der „American Academy of Orthopaedic Surgeons“, dem "Council of Musculoskeletal Specialty Societies“ und dem „Institute for Work and Health“ als Messinstrument für die Erfassung von Behinderungen nach Verletzungen an der oberen Extremität entwickelt worden (deutsche Übersetzung in Anlage 1).

Die Besonderheit dieses Fragenkatalogs ist, dass neben funktionellen und anatomischen Kriterien zur Erfassung der unmittelbaren Schädigung und ihrer Folgen auch die Wiedereingliederung in das soziale und berufliche Umfeld thematisiert wird. In drei Abschnitten werden Angaben zu Funktion, Symptomatik und speziellen Aktivitäten abgefragt. Die Tatsache, dass die Erfassung der Daten auf der subjektiven Wahrnehmung und Mitteilung des Patienten selbst beruht, stellt dabei keinen grundsätzlichen Mangel dar.

Diesem Blickwinkel liegt die Hypothese zugrunde, dass es der Patient selbst ist, der am besten einschätzen kann, ob er die von ihm erwarteten Leistungsanforderungen erfüllen kann und in der Lage ist, alltäglichen Routinetätigkeiten durchzuführen. Wird die Selbsteinschätzung des 
Patienten ernst genommen, kann die Rehabilitation des Patienten im Sinne einer Wiedereingliederung in sein berufliches und soziales Umfeld individuell angepasst und optimiert werden.

Die Antworten unserer Patienten ergaben, dass die vom DASHFragebogen erfragten Beeinträchtigungen bei leichteren Tätigkeiten und Aktivitäten überwiegend nur geringfügig waren. Demgegenüber waren Beschwerdangaben bei schwereren oder anspruchsvolleren Tätigkeiten und Aktivitäten bei der Hälfte der befragten Patienten vorhanden. Für einige Tätigkeiten gaben bis zu einem Drittel der Patienten wenigstens merkliche Schwierigkeiten an. Diese Trends sind grundsätzlich so zu erwarten und stützen daher einerseits die Plausibilität des DASH-Fragebogens und seiner Aussage sowie andererseits die Eignung unseres Patientenkollektivs für die Bearbeitung der gewählten Fragestellung.

\subsection{Nürnberger Handscore}

Der DASH-Score ist sehr allgemein gehalten und berücksichtigt Besonderheiten von Verbrennungspatienten nicht ausreichend. Daher haben wir einen eigenständigen Fragebogen erarbeitet, um differenziertere Aussagen erhalten zu können.

Datengrundlage ist die Erfassung aller Patienten, die wegen Handverbrennungen in den Jahren 2006 und 2007 in unserer Intensiveinheit für Schwerbrandverletzte behandelt werden sind. Die erreichte Gesamtzahl von 81 Patienten und die unterschiedliche Ausprägung der Verletzungsschwere bilden ein Kollektiv, das für die Bearbeitung der Fragestellung ausreichend gut geeignet ist. Die erfreulich hohe Rücklaufquote von $100 \%$ stützt diese Einschätzung weiter. Sie ist teilweise auch Folge des relativ kurzen Nachbeobachtungszeitraums von ca. 1,8 Jahren. Da aber anzunehmen ist, dass über den Effekt der Gewöhnung mit zunehmender Zeit nach dem Unfall die Selbsteinschätzung der betreffenden Patienten eher günstiger werden wird, stellt der kurze Nachbeobachtungszeitraum sogar einen gewissen Vorteil dar. 
Der Fragebogen zum Nürnberger Handscore umfasst 20 Fragen, die sich auf das subjektive Empfinden des Betroffenen hinsichtlich des ästhetischen und funktionellen Ergebnisses nach abgeschlossener Behandlung der Handverbrennung beziehen. Sowohl die soziale (Beruf) wie auch die psychische (Gestik) Rollenerfüllung der Hände im Alltag wird abgefragt. Die physischen Verbrennungsfolgen werden hinsichtlich der optischen Erscheinung, des Sensibilitäts - und Funktionsverlustes sowie mit Bezug auf Schmerzen erfaßt. Das Schmerzempfinden wird besonders sorgfältig betrachtet und sowohl in einer verbalen und wie auch in einer numerischen Bewertungsskala erfragt. Außerdem wird Schmerz im Zusammenhang mit der Gelenkbeweglichkeit erfragt. Antwortmöglichkeiten zu diesen 20 Fragen sind entweder Freitext, Ja/Nein-Entscheidungen oder die Angabe einer einzig richtigen Antwort von bis zu vier vorgegebenen Möglichkeiten. Einige Fragen ermöglichen Antworten in Analogie zum Schulnotensystem und erlauben so differenzierte Angaben.

Da ein einfacher Vergleich der beiden Fragebögen durchgeführt werden sollte, wurde auch für die Berechnung des NFH-Scores eine Bandbreite zwischen 0 und 100 angestrebt. Ebenso wie beim DASH-Score sollten 0 Punkte einem sehr guten und 100 Punkte dem ungünstigsten denkbaren Ergebnis entsprechen.

Der DASH-Fragebogen bietet für jede seiner 30 Fragen jeweils fünf Antwortmöglichkeiten an, die dementsprechend einem oder mehreren, insgesamt bis zu fünf Punkten entsprechen. Das ist beim NFH anders. Will man die beiden Fragebögen miteinander vergleichen, ist es zunächst sinnvoll, die unterschiedlichen Antwortmöglichkeiten des Nürnberger Fragebogens so zu modifizieren, dass auch hier Antworten möglich werden, die zwischen einem und fünf Punkten entsprechen. Anders als beim DASH-Fragebogen kommen im NFH aber Fragen vor, die denknotwendig nur zwei Antworten erlauben oder auch Freitexteingaben ermöglichen. Dementsprechend waren zunächst Umformungen nötig. Letztlich konnte diese Umformungen für 20 Items so definiert werden, dass 
niedrige Punktwerte immer einem besonders günstigen und hohe Punktwerte einem besonders schlechten Ergebnis entsprachen.

Die statistische Überprüfung der so entstandenen Items ergab eine hohe Zuverlässigkeit der Skala. Außerdem konnten wir nachweisen, dass die einzelnen Items in hohem Maße miteinander korrelieren (Cronbach's Alpha $0,8)$. Somit haben wir uns dazu entscheiden können, tatsächlich sämtliche 20 Fragen in der weiteren Bearbeitung mit zu erfassen.

Die weitere Bearbeitung erfolgte mittels einer Summenformel in sehr ähnlicher Weise wie auch beim DASH.

\subsection{Interpretation}

Die Einführung eines neuen Scoringsystems ist dann berechtigt und sinnvoll, wenn es differenziertere Aussagen erlaubt als bereits etablierte Systeme. Es ist nicht ausreichend, lediglich gleichartige Informationen zu liefern wie jene.

Was muss man von einem sinnvollen Scoringsystem grundsätzlich erwarten dürfen?

1. Scores müssen in ihrer jeweiligen Bewertungsskala sinnvolle und plausible Ergebnisse bringen.

2. Scores sollten semiquantitativ interpretierbar sein. Differenzen zwischen ermittelten Scores sollen andersartigen realen Zuständen entsprechen. Nach Möglichkeiten sollen die numerischen Differenzen bei niedrigen Punktwerten ebenso sinnvoll sein wie bei hohen. Gleichzeitig sollen klinisch relevante Unterschiede sich tatsächlich auch in unterschiedlichen Scorewerten abbilden lassen.

Die empirisch gesicherte Tatsache, wonach die Verletzungstiefe einer Handverbrennung die Schwere der Folgezustände determiniert, soll sich in einem entsprechenden Verhalten der Scorewerte wiederspiegeln. An diesem einfachen Zusammenhang kann zunächst die Frage nach der Plausibilität eines Scoringsystems überprüft werden. 
Würden diese ergeben, dass ihr Betrag von der Geschlechtszugehörigkeit oder vom Alter abhängt, wäre inre Sinnhaftigkeit anzuzweifeln. Auch sollte der Scorewert unabhängig von der jeweiligen Unfallursache sein und die Frage, ob ein Arbeitsunfall vorlag, sollte keinerlei Auswirkungen haben.

Grundsätzlich kann man ferner annehmen, dass eine schwerere Verletzung tendenziell zu einem längeren Intensivaufenthalt und auch insgesamt zu einer längeren stationären Behandlung führt. Häufig sind schwerere Verbrennungsverletzungen an der Hand auch mit einer großflächigen Verbrennung der Körperoberfläche assoziiert. Ferner ist die Tiefe der Verbrennungswunde an der Hand von großer Bedeutung für die Folgeschäden. Und letztlich deutet die Notwendigkeit, mehrfach operieren zu müssen, auf einen ungünstigeren Folgezustand hin. Bei statistischen Tests ist von einem sinnvollen Scoringsystem also zu erwarten, dass sich signifikante geschlechtsspezifische, altersabhängige, ursachenbezogene oder versicherungstechnische Einflüsse nicht zeigen, während andererseits ein Zusammenhang zwischen Behandlungsdauer, Flächenausdehnung der Gesamtverbrennung, Tiefenausdehnung der Verletzung an der Hand und auch Zahl der Eingriffe signifikant nachgewiesen werden sollte.

Dies konnten wir in unseren entsprechenden statistischen Tests sowohl für den DASH-Score wie auch für den NFH-Score genauso bestätigen. Beide Systeme liefern inhaltlich plausible Aussagen.

Bei so viel scheinbarer Gleichförmigkeit bleibt aber dennoch ein wichtiger und letztlich entscheidender Unterschied zwischen den beiden Systemen: Der DASH-Score kann die zweite Anforderung, nämlich Grundlage für eine semiquantitative Bewertung zu sein, nicht erfüllen. Aus Abb. 24 ergibt sich anschaulich, dass es zahlreiche Fälle gibt, die mit dem NFH-Score unterschiedlich bewertet werden können, beim DASH-Score aber immer wieder nur den Wert 0 ergeben. Es handelt sich um eher geringgradige Verletzungsfolgen, deren Ursache also auch die weniger tiefreichenden Brandverletzungen an der Hand waren. Die alleinige Betrachtung der 38 Fälle mit ausschließlich oberflächlich zweitgradiger Verbrennung zeigt 
schlüssig, dass der NFH-Score hier in der differenzierten Bewertung deutlich überlegen ist (S. Abb. 26). Der DASH-Score nutzt die theoretisch bestehende Bandbreite 0-100 in unserem Kollektiv nicht aus. Dies leistet der NFH-Score deutlich besser (s. Abb. 25).

Der NFH-Score liefert also nicht nur gleichartige Ergebnisse. Statistisch sind die NFH- und DASH-Scorewerte sogar hochsignifikant verschieden. Vielmehr erlaubt er weitere, über den DASH-Score hinausgehende Aussagen. Seine Berechtigung erhält er dadurch, dass er Verletzungsfolgen von Patienten nach oberflächlich zweitgradigen Verbrennungen der Hand, die nicht operiert werden mussten, differenzierter abbilden kann. Seine Ergebnisse können semi-quantitativ interpretiert werden.

Bemerkenswert ist, dass der NFH-Score diese höhere Aussagekraft hat, obwohl er mit 20 Items eine deutlich kleinere Datenbasis hat als der DASHScore mit 30 Items. Ursächlich ist möglicherweise die starke Betonung subjektiver Kriterien beim Nürnberger Fragebogen, wodurch die Eigenbewertung der betroffenen Patienten ein besonders hohes Gewicht erhält. Ein anderer Grund für diese hohe Treffsicherheit liegt aber möglicherweise darin begründet, dass die Patienten, an denen dieser Score „erprobt“ wurde, dieselben sind, die mit ihren Antworten zum Nürnberger Fragebogen die Grundlage für die Erstellung des Scores gegeben haben. Hier ist wohl ein methodischer Bias gegeben.

\subsection{Ausblick}

Zunächst hatten wir den Fragebogen ausschließlich inhaltsbezogen gestaltet. Seine Form richtete sich noch nicht primär nach den Anforderungen für die weitere statistische Bearbeitung. Aufgrund der anschließenden Überlegungen im Zusammenhang mit unserer Auswertung wissen wir nun aber, dass gewisse Modifikationen sinnvoll sind. So kann zukünftig die Auswertung dadurch erleichtert werden, dass bei der Antwortmöglichkeit „Schulnotensystem“ unmittelbar auf das System „1 bis 5“ gewechselt wird. 
Auch kann insoweit eine Anpassung erfolgen, als nur noch nach einer einzigen Altersangabe gefragt werden muss. Ebenso ist die Schmerzangabe nach der VAS verzichtbar.

In der Literatur findet man eine große Anzahl von Arbeiten über Auswirkungen von Verbrennungsverletzungen an der Hand, die Angaben zu aktiver oder passiver Gelenkbeweglichkeit, Kraftentfaltung und Nervenfunktion machen, aber keine Korrelation zur globalen Handfunktion herstellen [34]. Ein Instrument wie der Nürnberger Fragebogen ist dazu geeignet, diese Lücke zu füllen.

Zusammenfassend liegt die besondere Bedeutung des neuen Scores darin, sensibler erkennen zu können, ob Patienten mit zunächtst weniger schwerwiegenden Verletzungsformen Spätfolgen haben, die im DASH gar nicht zu einer meßbaren Punktzahl führen. Sie könnten also auch bei Anwendung dieses ansonsten ja breit akzeptierten Instrumentes schlicht übersehen werden. Wenn man bedenkt, dass bereits kleine Korrekturen nach Handverbrennungen für die Betroffenen großen Nutzen haben können, wäre dies ein bedauerliches Versäumnis. 


\section{Zusammenfassung}

Um behandlungsfähige Folgeschäden nach Verbrennungsverletzungen an der Hand zu erkennen, sind Nachuntersuchungen erforderlich, die viele Patienten allerdings nicht wahrnehmen wollen oder können. Um wenigstens einen Anhalt über den jeweiligen Zustand zu gewinnen, kann man Befragungen mit standardisierten Katalogen, wie etwa dem DASHFragebogen, durchführen. Dieser ist allerdings allgemein auf Folgeschäden an der oberen Extremität ausgerichtet und hat daher nicht das Ziel, die Besonderheiten der Folgen von Verbrennungsverletzungen an der Hand zu erfassen. Der DASH-Score kann also keine differenzierten Aussagen zu derartigen Folgezuständen leisten.

Wir haben anhand der Befragung von 81 Patienten, die in den Jahren 2006 und 2007 wegen Handverbrennungen in unserer Behandlung waren, einen von uns neu entwickelten Fragenkatalog (Nürnberger Fragebogen Handverbrennung, NFH) erprobt und daraus einen neuartigen Koeffizienten (NFH-Score) errechnet, der die besonderen Anforderungen an ein ScoringSystem nach Verbrennungen an der Hand erheblich besser erfüllen kann als der DASH-Score. Mit entsprechenden statistischen Testverfahren lässt sich belegen, dass der NFH-Score bei der Bewertung von Verbrennungsfolgen besonders nach weniger schwerwiegenden Verbrennungsverletzungen dem DASH-Score deutlich überlegen ist. Daher, und auch wegen der einfachen Praktikabilität, empfehlen wir die Anwendung dieses Verfahrens besonders in den Fällen, in denen Patienten zu einer Nachuntersuchung nicht erscheinen können. 


\section{Literaturverzeichnis}

1. Baker SP, O'Neill B, Haddon W, Jr., Long WB. The injury severity score: a method for describing patients with multiple injuries and evaluating emergency care. J Trauma 1974; 14 (3):187196

2. Bannasch $H$, Stark GB. Sekundäre plastisch-rekonstruktive Maßnahmen. In: Wappler F, Spilker G (eds.), Verbrennungsmedizin. Stuttgart: Thieme. 2009: 185-196

3. Borman $\mathrm{H}$, Kostakoglu $\mathrm{N}$. Hand burns in young patients--loss of selfsufficiency and economic productivity. Ann.Plast Surg. 1998; 41 (3):335

4. Brenner $P$, Reichert $B$, Berger A. Replantationen bei Mehrfachverletzungen? Handchir.Mikrochir.Plast Chir. 1995; 27:12-16

5. Bruck JC. Begutachtung. In: Bruck JC, Müller FE, Steen M (eds.), Handbuch der Verbrennungstherapie. Landsberg: ecomed. 2002: 430-433

6. Busche MN, Herold C, Schedler A, Knobloch K, Vogt PM, Rennekampff HO. Der Biobrane-Handschuh bei Verbrennungen der Hand. Bewertung des funktionellen und kosmetischen Ergebnisses und Kostenvergleich zur konventionellen Therapie. Handchir Mikrochir Plast Chir 2009; 41 (6):348-354

7. Büttemeyer R, Steen M, Henkel VD, Germann G. Establishing a baseline for organisation and outcome in burn care-basic data compiled by German burn centres, 1991-2000. Burns 2004; $30(2): 115-120$

8. Cartotto R. The burned hand: optimizing long-term outcomes with a standardized approach to acute and subacute care. Clin.Plast Surg. 2005; 32 (4):515-27, vi

9. Cazzola M, Malcovati L. Prognostic classification and risk assessment in myelodysplastic syndromes. Hematol.Oncol.Clin.North Am. 2010; 24 (2):459-468

10. Changulani M, Okonkwo U, Keswani T, Kalairajah Y. Outcome evaluation measures for wrist and hand: which one to choose? Int.Orthop. 2008; 32 (1):1-6 
11. Cil Y, Eski M, Isik S. First dorsal metacarpal artery adipofascial flap for thenar burn contracture releasing. Burns 2008; 34 (1):127130

12. de Roche R. Epidemiologie. In: Bruck JC, Müller FE, Steen M (Hrsg.), Handbuch der Verbrennungstherapie. Landsberg: ecomed. 2002: 27-33

13. Deb R, Giessler GA, Przybilski M, Erdmann D, Germann G. Die plastisch-chirurgische Sekundärrekonstruktion von Schwerbrandverletzten. Chirurg 2004; 75 (6):588-598

14. Edlich RF, Larkham N, O'Hanlan JT, Berry R, Hiebert J, Rodeheaver GT, Edgerton MT. Modification of the American Burn Association injury severity grading system. JACEP. 1978; 7 (6):226-228

15. Fukunishi I. Relationship of cosmetic disfigurement to the severity of posttraumatic stress disorder in burn injury or digital amputation. Psychother.Psychosom. 1999; 68 (2):82-86

16. Gabbiani G, Ryan GB, Majne G. Presence of modified fibroblasts in granulation tissue and their possible role in wound contraction. Experientia 1971; 27 (5):549-550

17. Gauglitz GG, Herndon DN, Kamolz LP, Jeschke MG. Die Pathophysiologie von Verbrennungswunden. In: Kamolz LP, Herndon DN, Jeschke MG (Hrsg.), Verbrennungen: Diagnose, Therapie und Rehabilitation des thermischen Traumas. Wien: Springer. 2009: 5-23

18. Germann G, Wind G, Harth A. Standardisierung und Validierung der deutschen Version 2.0 des "Disability of Arm, Shoulder, Hand" (DASH)-Fragebogens zur Outcome-Messung an der oberen Extremität. Handchir Mikrochir Plast Chir 1999; 31 (3):149152

19. Gregoire E, Hoti E, Gorden DL, de la SS, Pascal G, Azoulay D. Utility or futility of prognostic scoring systems for colorectal liver metastases in an era of advanced multimodal therapy. Eur.J Surg.Oncol. 2010; 36 (6):568-574

20. Grigg-Damberger MM. The AASM scoring manual: a critical appraisal. Curr.Opin.Pulm.Med 2009;

21. Grishkevich VM. Postburn hand border contractures and eliminating them with trapeze-flap plasty. J Burn Care Res. 2010; 31 (2):286-291 
22. Hartmann B, Ottomann C. Chirurgische Therapie von Erwachsenen. In: Kamolz LP, Herndon DN, Jeschke MG (Hrsg.), Verbrennungen: Diagnose, Therapie und Rehabilitation des thermischen Traumas. Wien: Springer. 2009: 73-87

23. Haslik W, Kamolz LP, Lumenta DB, Hladik M, Beck H, Frey M. The treatment of deep dermal hand burns: How do we achieve better results? Should we use allogeneic keratinocytes or skin grafts? Burns 2010; 36 (3):329-334

24. Henckel von Donnersmarck G. Verbrennungen, München: Zuckschwerdt. 1998

25. Henckel von Donnersmarck G, Hörbrand F. Bestimmung der MdE für Brandverletzte. Jahrbuch für Versicherungsmedizin 1995;

26. Henne Bruns D, Dürig M, Kremer B. Chirurgie, Stuttgart: Thieme. 2003

27. Hörbrand F, Mühlbauer W, Henckel von Donnersmarck G. Zustand, Lebensweise und psychologische Aspekte von Brandverletzten. Jahrbuch für Versicherungsmedizin 1995; 99

28. Jackson D. The diagnosis of the depth of burning. Br.J Surg. 1953; 40 (164):588-596

29. Kitzinger HB, Karle B, Frey M. Verbrennungen der Hand. In: Kamolz LP, Herndon DN, Jeschke MG (Hrsg.), Verbrennungen: Diagnose, Therapie und Rehabilitation des thermischen Traumas. Wien: Springer. 2009: 95-108

30. Klose R. Verbrennungen und Hitzeschäden. In: Scholz J, Sefrin P, Böttiger BW, Dörges V, Wenzel V (Hrsg.), Notfallmedizin. Stuttgart: Georg Thieme Verlag. 2008: 374-387

31. Knaus WA, Zimmerman JE, Wagner DP, Draper EA, Lawrence DE. APACHE-acute physiology and chronic health evaluation: a physiologically based classification system. Crit Care Med 1981; 9 (8):591-597

32. Köhler L, Mennigen R, Eypasch E, Troidl H. Colitis ulcerosa: Lebensqualität nach operativer Therapie. Dtsch.Med Wochenschr. 1991; 116:1362-1367

33. Kornbluth J, Bhardwaj A. Evaluation of Coma: A Critical Appraisal of Popular Scoring Systems. Neurocrit.Care 2010;

34. Kowalske K. Outcome assessment after hand burns. Hand Clin. 2009; 25 (4):557-561 
35. Kraemer MD, Jones T, Deitch EA. Burn contractures: incidence, predisposing factors, and results of surgical therapy. J Burn Care Rehabil. 1988; 9 (3):261-265

36. Kramer A, Bruck JC, Eisenbeiss W, Meierhans R, Rudolph P. Hygiene an einer Intensivstation für Brandverletzte. In: Bruck JC, Müller FE, Steen M (Hrsg.), Handbuch der Verbrennungstherapie. Landsberg: ecomed. 2010: 51-64

37. Küchler T, Bullinger M. Onkologie. In: Ravens-Sieberer U, Cieza A (Hrsg.), Lebensqualität und Gesundheitsökonomie in der Medizin: Konzepte, Methoden, Anwendungen. Landsberg: ecomed. 2000: 144-158

38. Lackner CK, Widmann JH, Ruppert M. Verbrennung, Verbrühung. In: Dick W (Hrsg.), Notfall- und Intensivmedizin. Berlin, New York: Walter de Gruyter. 2001: 156-162

39. Luce EA. The acute and subacute management of the burned hand. Clin.Plast Surg. 2000; 27 (1):49-63

40. Mazzetto-Betti KC, Amancio AC, Farina JA, Jr., Barros ME, Fonseca MC. High-voltage electrical burn injuries: functional upper extremity assessment. Burns 2009; 35 (5):707-713

41. Mustoe TA, Cooter RD, Gold MH, Hobbs FD, Ramelet AA, Shakespeare PG, Stella M, Teot L, Wood FM, Ziegler UE. International clinical recommendations on scar management. Plast Reconstr.Surg. 2002; 110 (2):560-571

42. Oestern HJ, Tscherne $H$, Sturm JA. Klassifizierung der Verletzungsschwere. Unfallchirurg 1985; 88:465-472

43. Pallua N. Thermische Verletzungen. In: Berger A (Hrsg.), Plastische Chirurgie-Handchirurgie. München: Urban \& Schwarzenberg. 1997: 59-66

44. Pallua N, Kunsebeck HW, Noah EM. Psychosocial adjustments 5 years after burn injury. Burns 2003; 29 (2):143-152

45. Pallua N, Markowicz M. Primär plastisch-chirurgische Therapie. In: Wappler F, Spilker G (Hrsg.), Verbrennungsmedizin. Stuttgart: Thieme. 2009: 41-54

46. Pawlik TM, Poon RT, Abdalla EK, Zorzi D, Ikai I, Curley SA, Nagorney DM, Belghiti J, Ng IO, Yamaoka Y, Lauwers GY, Vauthey JN. Critical appraisal of the clinical and pathologic predictors of survival after resection of large hepatocellular carcinoma. Arch.Surg. 2005; 140 (5):450-457 
47. Rennekampff HO, Berger A. Pathophysiologie der Verbrennungswunde. In: Bruck JC, Müller FE, Steen M (Hrsg.), Handbuch der Verbrennungstherapie. Landsberg: ecomed. 2002: 43-50

48. Riede UN, Schäfer HE. Physikalische Zellschädigung. In: Riede UN, Schäfer HE (Hrsg.), Allgemeine und Spezielle Pathologie. Stuttgart: Thieme. 1993: 155-168

49. Schmidt HM, Lanz U. Chirurgische Anatomie der Hand, Stuttgart: Thieme. 2003

50. Schneider JC, Holavanahalli R, Helm P, O'Neil C, Goldstein R, Kowalske K. Contractures in burn injury part II: investigating joints of the hand. J Burn Care Res. 2008; 29 (4):606-613

51. Smith DJ, McHugh TP, Phillips LG, Robson MC, Heggers JP. Biosynthetic compound dressings - management of hand burns. Burns 1988; 14:405-408

52. Steen M. Die verbrannte Hand. In: Krupp S, Rennekampff HO, Pallua N (Hrsg.), Plastische Chirurgie. Klinik und Praxis. Landsberg: ecomed. 2006: 2.1

53. Steen, M. Statistik der Deutschsprachigen Arbeitsgemeinschaft für Verbrennungsbehandlung. Persönliche Mitteilung, 2010

54. Sterling J, Gibran NS, Klein MB. Acute management of hand burns. Hand Clin. 2009; 25 (4):453-459

55. Sykes PJ. Severe burns of the hand: a practical guide to their management. J.Hand Surg.[Br.] 1991; 16:6-12

56. Tanttula K, Vuola J, sko-Seljavaara S. Return to employment after burn. Burns 1997; 23 (4):341-344

57. Teasdale G, Jennett B. Assessment of coma and impaired consciousness. A practical scale. Lancet 1974; 2 (7872):81-84

58. Tobiasen J, Hiebert JM, Edlich RF. The abbreviated burn severity index. Ann.Emerg.Med 1982; 11 (5):260-262

59. van Zuijlen PP, Kreis RW, Vloemans AF, Groenevelt F, Mackie DP. The prognostic factors regarding long-term functional outcome of full-thickness hand burns. Burns 1999; 25 (8):709-714

60. Vogt PM, Ipaktchi K. Pathophysiologie der Verbrennungskrankheit. In: Wappler F, Spilker G (Hrsg.), Verbrennungsmedizin.

Stuttgart: Thieme. 2009: 3-14 
61. Vogt, P. M., Mailänder, P., Jostkleigrewe, F., Reichert, B., and Adams, H. A. Zentren fur Schwerbrandverletzte in der Bundesrepublik Deutschland--Versorgungsstrukturen und Bedarf. Chirurg Suppl, 411-413. 2007.

62. Vogt PM, Mühlberger T, Drücke D, Steinau HU. Thermische, chemische und elektrische Schäden. In: Bruch HP, Trentz O (Hrsg.), Chirurgie. München: Urban \& Fischer Verlag. 2001: 447-460

63. Wallace AB. The exposure treatment of burns. Lancet $1951 ; 1: 501-$ 504

64. Woo SH, Seul JH. Optimizing the correction of severe postburn hand deformities by using aggressive contracture releases and fasciocutaneous free-tissue transfers. Plast Reconstr.Surg. $2001 ; 107(1): 1-8$

65. Zellweger G. Die Behandlung der Verbrennungen. Praktische Hinweise für Diagnose, Therapie, Rehabilitation, Köln: Deutscher Ärzteverlag. 1985 


\section{Anlagen}

\section{Anlage 1: DASH-Fragebogen}

Der vorliegende Fragebogen beschäftigt sich sowohl mit Ihren Symptomen als auch Ihren Fähigkeiten, bestimmte Tätigkeiten/Aktivitäten auszuführen. Bitte beantworten Sie alle Fragen gemäß Ihrem Zustand in der letzte Woche, indem Sie einfach die entsprechende Antwort ankreuzen. Es ist nicht entscheidend, mit welchem Arm oder welcher Hand Sie Ihre Tätigkeiten / Aktivitäten ausüben. Antworten Sie einfach entsprechend Ihren Fähigkeiten, egal, wie Sie die Arbeit meistern konnten.

Teil A

Bitte schätzen Sie Ihre Fähigkeiten ein, folgende Tätigkeiten/Aktivitäten in der letzten Woche ausführen zu können, indem Sie die entsprechende Zahl einkreisen.

\begin{tabular}{|c|c|c|c|c|c|}
\hline & $\begin{array}{l}\text { Keine } \\
\text { Schwierigkei }\end{array}$ & $\begin{array}{l}\text { Wenig } \\
\text { Schwierigkeiter }\end{array}$ & $\begin{array}{l}\text { Merkliche } \\
\text { Schwierigkeiten, } \\
\text { aber machbar }\end{array}$ & $\begin{array}{l}\text { Erhebliche } \\
\text { Schwierigkeiter }\end{array}$ & Jicht möglich \\
\hline 1. Ein Marmeladen-, Einmach-, Honigglas öffnen & 1 & 2 & 3 & 4 & 5 \\
\hline 2. Schreiben & 1 & 2 & 3 & 4 & 5 \\
\hline 3. Schlüssel umdrehen & 1 & 2 & 3 & 4 & 5 \\
\hline 4. Eine Mahlzeit zubereiten & 1 & 2 & 3 & 4 & 5 \\
\hline 5. Eine schwere Tür aufstoßen & 1 & 2 & 3 & 4 & 5 \\
\hline $\begin{array}{l}\text { 5. Etwas auf ein Regal über } \\
\text { Kopfhöhe stellen }\end{array}$ & 1 & 2 & 3 & 4 & 5 \\
\hline $\begin{array}{l}\text { 7. Schwere Hausarbeit (Boden } \\
\text { putzen, Wände abwaschen) }\end{array}$ & 1 & 2 & 3 & 4 & 5 \\
\hline 8. Garten- oder Hofarbeit & 1 & 2 & 3 & 4 & 5 \\
\hline 9. Betten machen & 1 & 2 & 3 & 4 & 5 \\
\hline $\begin{array}{l}\text { 10. Einkaufstüte oder } \\
\text { Aktenkoffer tragen }\end{array}$ & 1 & 2 & 3 & 4 & 5 \\
\hline 11. Schwere Gegenstände tragen & 1 & 2 & 3 & 4 & 5 \\
\hline 12. Eine Glühbirne über Kopf auswechseln & 1 & 2 & 3 & 4 & 5 \\
\hline 13. Haare waschen oder fönen & 1 & 2 & 3 & 4 & 5 \\
\hline 14. Den Rücken waschen & 1 & 2 & 3 & 4 & 5 \\
\hline 15. Einen Pullover anziehen & 1 & 2 & 3 & 4 & 5 \\
\hline $\begin{array}{l}\text { 16. Ein Messer benutzen, um } \\
\text { Lebensmittel zu schneiden }\end{array}$ & 1 & 2 & 3 & 4 & 5 \\
\hline $\begin{array}{l}\text { 17. Freizeitaktivitäten, die wenig körperliche } \\
\text { Anstrengung verlangen } \\
\text { (z.B. Karten spielen, stricken usw.) }\end{array}$ & 1 & 2 & 3 & 4 & 5 \\
\hline $\begin{array}{l}\text { 18. Freizeitaktivitäten, bei denen Sie Ihren Arm mit } \\
\text { mehr oder weniger Anstrengung benutzen, } \\
\text { wie z.B. Tennis, Heimwerken, Golfen }\end{array}$ & 1 & 2 & 3 & 4 & 5 \\
\hline $\begin{array}{l}\text { 19. Freizeitaktivitäten, bei denen Sie Ihren Arm frei } \\
\text { bewegen (Badminton, Frisbee, Squasch) }\end{array}$ & 1 & 2 & 3 & 4 & 5 \\
\hline $\begin{array}{l}\text { 20. Am Straßenverkehr teilnehmen oder öffentliche } \\
\text { Verkehrsmittel benutzen, um von einem Platz zum } \\
\text { zu gelangen }\end{array}$ & 1 & 2 & 3 & 4 & 5 \\
\hline 21. Sexuelle Aktivität & 1 & 2 & 3 & 4 & 5 \\
\hline $\begin{array}{l}\text { 22. In welchem Ausmaß haben Ihre Schulter-, Arm- } \\
\text { oder Handprobleme ihren normalen Kontakt zu } \\
\text { Familie, Freunden, Nachbarn oder anderen } \\
\text { Gruppen während der letzten Woche beeinflußt? }\end{array}$ & $\begin{array}{l}\text { Überhaupt } \\
\text { nicht } \\
\quad 1\end{array}$ & $\begin{array}{c}\text { Kaum } \\
2\end{array}$ & $\begin{array}{c}\text { Merklich } \\
3\end{array}$ & $\begin{array}{c}\text { Deutlich } \\
4\end{array}$ & $\begin{array}{c}\text { Extrem } \\
5\end{array}$ \\
\hline $\begin{array}{l}\text { 23. Waren Sie in der vergangenen Woche durch Ihre } \\
\text { Schulter-, Arm- oder Handprobleme in Ihrer Arbei } \\
\text { Oder anderen täglichen Aktivitäten eingeschränkt? }\end{array}$ & $\begin{array}{l}\text { Überhaupt } \\
\text { Nicht } \\
1\end{array}$ & $\begin{array}{l}\text { Kaum } \\
\text { eingeschränkt } \\
2\end{array}$ & $\begin{array}{l}\text { Merklich } \\
\text { eingeschränkt } \\
3\end{array}$ & $\begin{array}{l}\text { Deutlich } \\
\text { eingeschränkt } \\
\quad 4\end{array}$ & $\begin{array}{l}\text { Extrem } \\
\text { eingeschränkt } \\
\quad 5\end{array}$ \\
\hline
\end{tabular}


Teil B:

Bitte schätzen Sie die Schwere der folgenden Symptome während der letzten Woche ein. (Bitte kreisen Sie die entsprechende Zahl ein.)

\section{Schmerzen in Schulter, Arm, Hand}

25. Schmerzen in Schulter, Arm, Hand, nachdem Sie eine bestimmte Tătigkeit ausgeführt haben

26. Kribbeln (Nadelstiche) in Schulter, Arm, Hand

27. Schwächegefühl in Schulter, Arm, Hand

28. Steifheit in Schulter, Arm, Hand

29. Hatten Sie in der letzten Woche Schlafstörungen Kein Hand?

\begin{tabular}{|c|c|c|c|c|}
\hline Keine & Wenig & Mittel & Erheblich & Extrem \\
\hline 1 & 2 & 3 & 4 & 5 \\
\hline 1 & 2 & 3 & 4 & 5 \\
\hline 1 & 2 & 3 & 4 & 5 \\
\hline 1 & 2 & 3 & 4 & 5 \\
\hline 1 & 2 & 3 & 4 & 5 \\
\hline Keine & Wenig & Merkliche & Erhebliche & So viele \\
\hline Schwierigkeiten & Schwierigkeiten & Schwierigkeiten & Schwierigkeiten & $\begin{array}{l}\text { Schwierigkeiten, } \\
\text { dass ich nicht } \\
\text { schlafen konnte }\end{array}$ \\
\hline 1 & 2 & 3 & 4 & 5 \\
\hline $\begin{array}{l}\text { Stimme } \\
\text { Öberhaupt nicht } \\
\mathrm{zu}\end{array}$ & $\begin{array}{l}\text { Stimme } \\
\text { nicht } \mathrm{zu}\end{array}$ & $\begin{array}{l}\text { Weder Zustimmung } \\
\text { noch Ablehnung. } \\
\text { Weiß nicht }\end{array}$ & g Stimme zu & Stimme sehr zu \\
\hline 1 & 2 & 3 & 4 & 5 \\
\hline
\end{tabular}

Durch meine Probleme in Schulter, Arm oder

Hand fühle ich mich weniger fähig,

mein Selbstvertrauen ist eingeschränkt, und ich

kann mich weniger nützlich machen.

Oberhaupt nicht nicht 2

noch Ablehnung.

3

Teil C (optionaler Teil): Sport- oder Musikinstrumente

Die folgenden Fragen beziehen sich auf den Einfluß, den Ihr Schulter-, Arm-, oder Handproblem auf das Spielen Ihres Musikinstrumentes oder das Ausüben Ihres Sports hatte.

Wenn Sie mehr als ein instrument spielen oder mehr als eine Sportart ausüben, so geben Sie bitte an, welches Instrumet oder welche Sportart für Sie am wichtigsten ist.

Bitte kreisen Sie die Zahl ein, die Ihre körperlichen Fähigkeiten in der letzten Woche am besten beschreibt.

\begin{tabular}{|c|c|c|c|c|c|}
\hline Hatten Sie irgendwelche Schwierigkeiten: & $\begin{array}{l}\text { Keine } \\
\text { Schwierigkeiten }\end{array}$ & $\begin{array}{l}\text { Wenig } \\
\text { Schwierigkeiten }\end{array}$ & $\begin{array}{l}\text { Merkliche } \\
\text { Schwierigkeiten, } \\
\text { aber machbar }\end{array}$ & $\begin{array}{l}\text { Erhebliche } \\
\text { Schwierigkeiten }\end{array}$ & Nicht möglich \\
\hline $\begin{array}{l}\text { 1. In der üblichen Art und Weise, } \\
\text { Ihr Musikinstrument zu spielen } \\
\text { Oder Sport zu treiben? }\end{array}$ & 1 & 2 & 3 & 4 & 5 \\
\hline $\begin{array}{l}\text { 2. Wegen der Schmerzen in Schulter } \\
\text { in Schulter/ Arm/ Hand Ihr } \\
\text { Musikinstrument zu spielen oder } \\
\text { Sport zu treiben? }\end{array}$ & 1 & 2 & 3 & 4 & 5 \\
\hline $\begin{array}{l}\text { 3. So gut, wie Sie es gewohnt waren, } \\
\text { Ihr Musikinstrument zu spielen } \\
\text { oder Sport zu treiben? }\end{array}$ & 1 & 2 & 3 & 4 & 5 \\
\hline $\begin{array}{l}\text { 4. Ihre gewohnte Zeit mit dem } \\
\text { Spielen Ihres Musikinstrumentes } \\
\text { oder mit Sporttreiben zu verbringen? }\end{array}$ & 1 & 2 & 3 & 4 & 5 \\
\hline
\end{tabular}

Wir bedanken uns für Ihre Mitarbeit 
Anlage 2: Nürnberger Fragebogen Handverbrennung (NFH)

\section{Fragebogen}

1. Wie alt sind Sie?

2. Wie alt waren Sie zum Zeitpunkt des Unfalls?

3. Üben Sie Ihren damaligen Beruf heute noch aus?

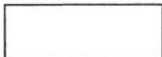

Hat sich die Verbrennung an den Händen auf Ihre berufliche Tätigkeit ausgewirkt?

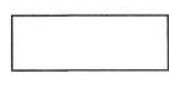

Ja

$\square \quad$ Nein

$\square$ Keine Einschränkung

$\square$ Leichte Einschränkung

$\square$ Starke Einschränkung

$\square$ Berufliche Tätigkeit ist durch die Handverbrennungen nicht mehr möglich

5. Wie bewerten Sie das durch die Operation/ Behandlung erzielte Ergebnis (Bewertung im Schulnotensystem: $1=$ sehr gut;

6=Mangelhaft)?

6. Wie bewerten Sie das Aussehen Ihrer Hände heute (Bewertung im Schulnotensystem: 1= sehr gut; $6=$ Mangelhaft)?

7. Verstecken Sie Ihre Hände vor der Offentlichkeit ?

8. Verwenden Sie Thre Hände zum Gestikulieren heute mehr oder weniger?

9. Sind Farbunterschiede im Vergleich zur gesunden Haut zu erkennen?

$\square$

$\square 6$

$\square$
$1 \square 2$

$\square 4$

$\square 5$

$\square$ Ja

$\square$ Nein

heute mehr als früher

$\square$ heute bewusst weniger als früher

betroffene Areale sind heller

betroffene Areale sind dunkler

betroffene Areale sind gleichfarbig wie die gesunde, umgebende Haut

ca. $\quad \%$

Sensibilität sind seit dem Unfall wiedergekehrt $(0 \%=$ völlig gefühllos, $100 \%=$ Gefühl wie vorher)

11. Haben Sie Schmerzen in den Händen?

a) Verbale Ratingskala

Verbale Ratingskala
Zutreffendes bitte ankreuzen

keine Schmerz

leichter Schmerz

mäßiger Schmerz

starker Schmerz

maximal vorstellbarer Schmerz 
b) Numerische Ratingskala

(Bitte schä̈zen Sie anhand der Zahl die Intensität Ihrer Schmerzen ein und kreuzen Sie eine entsprechende Zahl an; 0 bedeutet keine Schmerzen, 10 bedeutet die stärksten vorstellbaren Schmerzen)

$\begin{array}{lllllllllll}0 & 1 & 2 & 3 & 4 & 5 & 6 & 7 & 8 & 9 & 10\end{array}$

c) Visuelle Analogskala

(Bitte markieren Sie die für Sie entsprechende Schmerzintensität an der entsprechenden Stelle.)

\begin{tabular}{|l} 
\\
kein Schmerz \\
stärkster Schmerz
\end{tabular}

12. Die Kälteempfindlichkeit an den verbrannten Hautarealen der Hände ist

$\square$ erhöht

$\square$ vermindert

$\square$ unverändert

13. Die Wärmeempfindlichkeit an den verbrannten Hautarealen der Hände ist

$\square$ erhöht

$\square$ vermindert

$\square$ unverändert

14. Die Trockenheit der Haut an den verbrannten

$\square$ erhöht Hautarealen der Hände is

$\square$ vermindert

$\square$ unverändert

15. Die Verletzlichkeit der Haut an den

$\square$ erhöht

verbrannten Hautarealen der Hände ist

$\square$ vermindert

$\square$ unverăndert

$\begin{array}{ll}\text { 16. Es besteht Juckreiz an den verbrannten } & \square \text { Ja } \\ \text { Hautarealen der Hände } & \square \text { Nein } \\ \begin{array}{l}\text { 17. Es besteht ein Taubheitsgefühl an den } \\ \text { verbrannten Hautarealen der Hände }\end{array} & \square \quad \text { Ja } \\ \begin{array}{l}\text { 18. Es besteht Spannungsgefühl an den } \\ \text { verbrannten Hautarealen der Hände }\end{array} & \square \text { Ja } \\ \begin{array}{l}\text { 19. Die Schweißneigung der Hände ist seit der } \\ \text { Verbrennung }\end{array} & \square \text { erhöht } \\ & \square \text { vermindert } \\ \begin{array}{l}\text { 20. Es bestehen Gelenk-/ Gliederschmerzen an } \\ \text { den Händen seit der Verbrennung }\end{array} & \square \text { Ja unverändert } \\ & \square \text { Nein }\end{array}$




\section{Anlage 3: Fragenkatalog mit Bewertungsschema zum Nürnberger Handscore}

\begin{tabular}{|c|c|c|c|c|c|}
\hline & 1 & 2 & 3 & 4 & 5 \\
\hline $\begin{array}{ll}1 & \text { Lebensalter des Patienten zum } \\
\text { Unfallzeitpunkt [Jahre] }\end{array}$ & $<27,5$ & - & $\begin{array}{l}27,5 \leq x \\
\geq 50,5\end{array}$ & - & $>50,5$ \\
\hline $\begin{array}{l}2 \text { Wieder im ursprünglichen Beruf } \\
\text { tätig? }\end{array}$ & Ja & - & Nein & - & Kein Beruf \\
\hline $\begin{array}{l}3 \text { Wirken sich die Verletzungsfolgen } \\
\text { auf die berufliche Tätigkeit aus? }\end{array}$ & nein & leicht & stark & $\begin{array}{l}\text { sehr } \\
\text { stark }\end{array}$ & berufsunfähig \\
\hline $\begin{array}{l}4 \text { Subjektive Bewertung erzielten } \\
\text { Behandlungsergebnisses }\end{array}$ & $\begin{array}{c}\text { sehr } \\
\text { gut }\end{array}$ & - & gut & - & $\begin{array}{l}\text { befriedigend } \\
\text { und } \\
\text { schlechter }\end{array}$ \\
\hline $\begin{array}{l}5 \text { Subjektive Bewertung des } \\
\text { Aussehens der Hände }\end{array}$ & $\begin{array}{c}\text { sehr } \\
\text { gut }\end{array}$ & - & gut & - & $\begin{array}{l}\text { befriedigend } \\
\text { und } \\
\text { schlechter }\end{array}$ \\
\hline $\begin{array}{l}6 \text { Werden die Hände in der } \\
\text { Öffentlichkeit versteckt? }\end{array}$ & nein & - & - & - & ja \\
\hline $\begin{array}{l}7 \text { Werden die Hände zum } \\
\text { Gestikulieren verwendet? }\end{array}$ & $\begin{array}{l}\text { mehr } \\
\text { als } \\
\text { früher }\end{array}$ & - & - & - & weniger a.f. \\
\hline 8 Gibt es Farbunterschiede? & nein & - & - & - & ja \\
\hline $\begin{array}{l}9 \text { Sensibilitätsgrad }(100 \%= \\
\text { ungestört) }\end{array}$ & $100 \%$ & - & $3-99 \%$ & - & unter $73 \%$ \\
\hline $10 \begin{array}{l}\text { Bestehen Schmerzen? } \\
\text { (Bewertung verbal) }\end{array}$ & keine & leichte & mäßige & starke & maximale \\
\hline 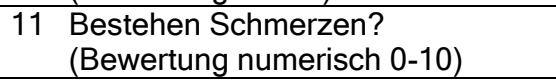 & 0 & - & 1 & - & 2 und mehr \\
\hline 12 Besteht eine Kälteempfindlichkeit? & nein & - & - & - & ja \\
\hline $\begin{array}{l}13 \text { Besteht eine } \\
\text { Wärmeempfindlichkeit? }\end{array}$ & nein & - & - & - & ja \\
\hline 14 Ist die Haut zu trocken? & nein & - & - & - & ja \\
\hline 15 Ist die Haut besonders verletzlich? & nein & - & - & - & ja \\
\hline 16 Besteht Juckreiz? & nein & - & - & - & ja \\
\hline 17 Besteht ein Taubheitsgefühl? & nein & - & - & - & ja \\
\hline 18 Besteht ein Spannungsgefühl? & nein & - & - & - & ja \\
\hline 19 Ist die Schweißneigung verstärkt? & nein & - & - & - & ja \\
\hline $\begin{array}{l}\text {-20 Bestehen Gliederschmerzen an } \\
\text { der Hand? }\end{array}$ & nein & - & - & - & ja \\
\hline
\end{tabular}




\section{Danksagung}

Ich danke ...

... ganz besonders Herrn Privatdozent Dr. med. Dr. med. habil. Bert Reichert, der mein Doktorvater, Betreuer und Chefarzt in einer Person ist. Für die Möglichkeit der Durchführung dieser Dissertation in seiner Klinik, für die vielen Gespräche, den unermüdlichen Einsatz und die Bereitschaft, mir jederzeit bei allen theoretischen und methodischen Problemen hilfreich zur Seite zu stehen, sowie für den Einsatz bei der Durchführung und Ausarbeitung dieser Dissertation. Für die freundliche und ermutigende Art dabei bedanke ich mich vor allem.

... meinem Kollegen Dr. med. Dirk Wisser, für die anregenden Diskussionen zu Beginn dieser Dissertation, die zur Entstehung des Themas beigetragen haben.

... meiner Freundin Katrin Dittmann, für ihre Unterstützung bei der Ausarbeitung des Layouts des Fragebogens für den NFH score.

... allen brandverletzten Patienten, die an der Befragung teilgenommen haben, für ihre Offenheit und ihr Vertrauen sowie den Glauben daran, dass das Schlimme, das innen passiert ist, durch die Teilnahme an der Studie auch etwas Gutes bewirken kann.

... meinem Mann Michael für die intensive Beratung bei der Erstellung der Dissertation, die Korrekturen und das stetige Interesse, den Rückhalt, den Zuspruch und die Unterstützung, ohne die ich die Dissertation nicht hätte fertig stellen können.

... meinem Vater, der mir in jeder Situation mit Rat und Tat zur Seite stand und steht und über lange, teilweise aussichtslose Strecken hinweg immer an die Fertigstellung dieser Dissertation geglaubt hat.

Ich widme diese Arbeit meinen Eltern. 


\section{Lebenslauf}

Name:

Beate Maria Schneider (geb. Gonsior)

Geburtsdatum/ -ort:

Nationalität:

22.11.1975 in Hindenburg

deutsch

Familienstand:

verheiratet seit 31.07.2009

Anschrift:

Ortnitstraße 4; 81925 München

\section{Schulbildung}

1982-1986

1986-1995

Grundschule Burgholzhausen

Mai 1995

Phillip Reis Schule, Friedrichsdorf

Abitur

\section{Studium der Humanmedizin}

Okt. 1995 - Nov. 2002

Medizinische Hochschule Hannover

Nov. 2002

Approbation als Ärztin

\section{Ärztliche Tätigkeit}

08.04. 2003 - 31.10. 2004

Ärztin im Praktikum im

Bethanienkrankenhaus, Frankfurter

Diakonie-Kliniken, Abteilung für

Chirurgie ( Plastische Chirurgie, HNO

Chirurgie, Allgemeinchirurgie,

Gefäßchirurgie, operative

Gynäkologie).

$01.11 .2004-30.11 .2005$

Assistenzärztin im St.

Marienkrankenhaus, Frankfurt am

Main, Abteilung für Allgemein- und

Viszeralchirurgie, Prof. Dr. med. C.

Hottenrott

$01.12 .2005-30.11 .2007$

Assistenzärztin Plastische Chirurgie, Praxis für Plastische und Ästhetische Chirurgie, Prof. Dr. med. Dr. med. habil A.-M. Feller

Seit 01.01 .2008

Assistenzärztin Plastische Chirurgie, Klinik für Plastische, Widerherstellende und Handchirurgie, Zentrum für Schwerbrandverletzte, Klinikum Nürnberg, PD Dr. med. Dr. med. habil. Bert Reichert 